\title{
On the Core of a Low Dimensional Set-Valued Mapping
}

\author{
By Pavel Shvartsman \\ Department of Mathematics, Technion - Israel Institute of Technology, \\ 32000 Haifa, Israel \\ e-mail:pshv@technion.ac.il
}

\begin{abstract}
Let $\mathfrak{M}=(\mathcal{M}, \rho)$ be a metric space and let $X$ be a Banach space. Let $F$ be a set-valued mapping from $\mathcal{M}$ into the family $\mathcal{K}_{m}(X)$ of all compact convex subsets of $X$ of dimension at most $m$. The main result in our recent joint paper with Charles Fefferman (which is referred to as a "Finiteness Principle for Lipschitz selections") provides efficient conditions for the existence of a Lipschitz selection of $F$, i.e., a Lipschitz mapping $f: \mathcal{M} \rightarrow X$ such that $f(x) \in F(x)$ for every $x \in \mathcal{M}$.

We give new alternative proofs of this result in two special cases. When $m=2$ we prove it for $X=\mathbf{R}^{2}$, and when $m=1$ we prove it for all choices of $X$. Both of these proofs make use of a simple reiteration formula for the "core" of a set-valued mapping $F$, i.e., for a mapping $G: \mathcal{M} \rightarrow \mathcal{K}_{m}(X)$ which is Lipschitz with respect to the Hausdorff distance, and such that $G(x) \subset F(x)$ for all $x \in \mathcal{M}$.
\end{abstract}

\section{Introduction.}

Let $\mathfrak{M}=(\mathcal{M}, \rho)$ be a pseudometric space, i.e., suppose that the "distance function" $\rho: \mathcal{M} \times \mathcal{M} \rightarrow[0,+\infty]$ satisfies $\rho(x, x)=0, \rho(x, y)=\rho(y, x)$, and $\rho(x, y) \leq \rho(x, z)+\rho(z, y)$ for all $x, y, z \in \mathcal{M}$. Note that $\rho(x, y)=0$ may hold with $x \neq y$, and $\rho(x, y)$ may be $+\infty$.

Let $(X,\|\cdot\|)$ be a real Banach space. Given a non-negative integer $m$ we let $\mathcal{K}_{m}(X)$ denote the family of all non-empty compact convex subsets $K \subset X$ of dimension at most $m$. (We say that a convex subset of $X$ has dimension at most $m$ if it is contained in an affine subspace of $X$ of dimension at most $m$.) We let $\mathcal{K}(X)=\bigcup\left\{\mathcal{K}_{m}(X): m=0,1, \ldots\right\}$ denote the family of all non-empty compact convex finite-dimensional subsets of $X$.

$\operatorname{By} \operatorname{Lip}(\mathcal{M}, X)$ we denote the space of all Lipschitz mappings from $\mathcal{M}$ to $X$ equipped with the Lipschitz seminorm

$$
\|f\|_{\operatorname{Lip}(\mathcal{M}, X)}=\inf \{\lambda>0:\|f(x)-f(y)\| \leq \lambda \rho(x, y) \text { for all } x, y \in \mathcal{M}\} .
$$

In this paper we study the following problem.

Math Subject Classification: 46E35

Key Words and Phrases: Set-valued mapping, Lipschitz selection, Helly's theorem, the core of a set-valued mapping, Hausdorff distance, balanced refinement.

This research was supported by Grant No 2014055 from the United States-Israel Binational Science Foundation (BSF). 
Problem 1.1. Suppose that we are given a set-valued mapping $F$ which to each point $x \in \mathcal{M}$ assigns a set $F(x) \in \mathcal{K}_{m}(X)$. A selection of $F$ is a map $f: \mathcal{M} \rightarrow X$ such that $f(x) \in F(x)$ for all $x \in \mathcal{M}$.

We want to know whether there exists a selection $f$ of $F$ in the $\operatorname{space} \operatorname{Lip}(\mathcal{M}, X)$. Such an $f$ is called a Lipschitz selection of the set-valued mapping $F: \mathcal{M} \rightarrow \mathcal{K}_{m}(X)$.

If a Lipschitz selection $f$ exists, then we ask how small we can take its Lipschitz seminorm. See Fig. 1.

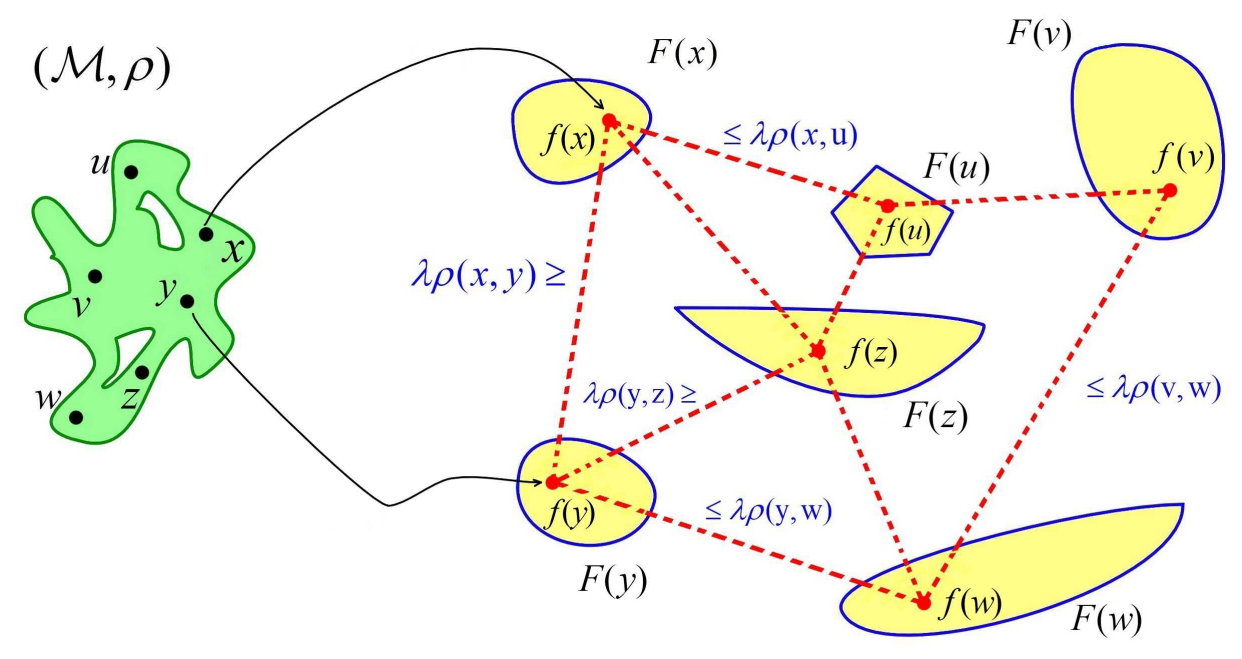

Fig. 1: $f: \mathcal{M} \rightarrow \mathbf{R}^{2}$ is a Lipschitz selection of the set-valued mapping $F: \mathcal{M} \rightarrow \mathcal{K}\left(\mathbf{R}^{2}\right)$.

The following result provides efficient conditions for the existence of a Lipschitz selection of an arbitrary set-valued mapping from a pseudometric space into the family $\mathcal{K}_{m}(X)$. We refer to it as a "Finiteness Principle for Lipschitz selections", or simply as a "Finiteness Principle".

Theorem 1.2. (Fefferman,Shvartsman [16]) Fix $m \geq 1$. Let $(\mathcal{M}, \rho)$ be a pseudometric space, and let $F: \mathcal{M} \rightarrow \mathcal{K}_{m}(X)$ for a Banach space $X$. Let

$$
N(m, X)=2^{\ell(m, X)} \quad \text { where } \quad \ell(m, X)=\min \{m+1, \operatorname{dim} X\} .
$$

Suppose that for every subset $\mathcal{M}^{\prime} \subset \mathcal{M}$ consisting of at most $N=N(m, X)$ points, the restriction $\left.F\right|_{\mathcal{M}^{\prime}}$ of $F$ to $\mathcal{M}^{\prime}$ has a Lipschitz selection $f_{\mathcal{M}^{\prime}}$ with Lipschitz seminorm $\left\|f_{\mathcal{M}^{\prime}}\right\|_{\operatorname{Lip}\left(\mathcal{M}^{\prime}, X\right)} \leq 1$.

Then $F$ has a Lipschitz selection $f$ with Lipschitz seminorm $\|f\|_{\operatorname{Lip}(\mathcal{M}, X)} \leq \gamma$ where $\gamma=\gamma(m)$ is a positive constant depending only $m$.

There is an extensive literature devoted to various versions of Finiteness Principles for Lipschitz selections and related topics. We refer the reader to the papers [1, 2, 4, 14, 16 , 19. 21, 23-26] and references therein for numerous results in this direction.

We note that the "finiteness number" $N(m, X)$ in Theorem 1.2 is optimal; see [24]. 
For the case of the trivial distance function $\rho \equiv 0$, Theorem 1.2 agrees with the classical Helly's Theorem [9], except that the optimal finiteness constant for $\rho \equiv 0$ is

$$
n(m, X)=\ell(m, X)+1=\min \{m+2, \operatorname{dim} X+1\} \quad \text { in place of } \quad N(m, X)=2^{\ell(m, X)} .
$$

Thus, Theorem 1.2 may be regarded as a generalization of Helly's Theorem.

Our interest in Helly-type criteria for the existence of Lipschitz selections was initially motivated by some intriguing close connections of this problem with the classical Whitney extension problem [27], namely, the problem of characterizing those functions defined on a closed subset, say $E \subset \mathbf{R}^{n}$, which are the restrictions to $E$ of $C^{m}$-smooth functions on $\mathbf{R}^{n}$. We refer the reader to the papers [5,-7, 10, 13, 26] and references therein for numerous results and techniques concerning this topic.

One of the main ingredients of the proof of Theorem 1.2 is the construction of a special set-valued mapping $G: \mathcal{M} \rightarrow \mathcal{K}_{m}(X)$ introduced in [16] which we call a "core" of the set-valued mapping $F$. In fact each core is associated with a positive constant. Here are the relevant definitions.

Definition 1.3. Let $\gamma$ be a positive constant, and let $F: \mathcal{M} \rightarrow \mathcal{K}_{m}(X)$ be a set-valued mapping. A set-valued mapping $G: \mathcal{M} \rightarrow \mathcal{K}_{m}(X)$ is said to be a $\gamma$-core of $F$ if

(i). $G(x) \subset F(x)$ for all $x \in \mathcal{M}$;

(ii). $G$ is $\gamma$-Lipschitz with respect to Hausdorff distance, i.e.,

$$
\mathrm{d}_{\mathrm{H}}(G(x), G(y)) \leq \gamma \rho(x, y) \quad \text { for all } \quad x, y \in \mathcal{M} \text {. }
$$

We refer to a map $G$ as a core of $F$ if $G$ is a $\gamma$-core of $F$ for some $\gamma>0$.

See Fig. 2, 3, 4.

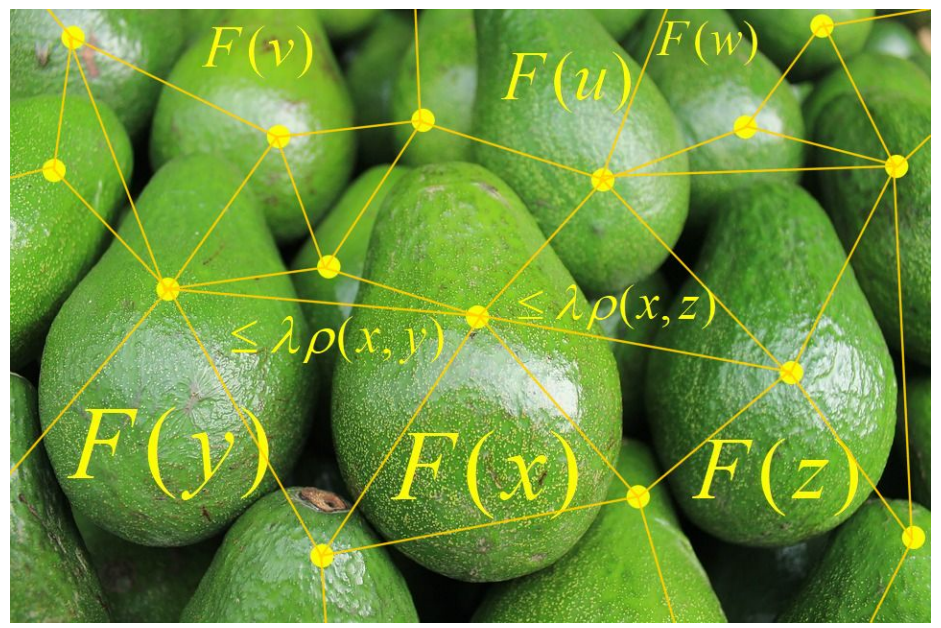

Fig. 2: A set-valued mapping $F$ into a family of avocados and its Lipschitz selection with Lipschitz seminorm at most $\lambda$. 


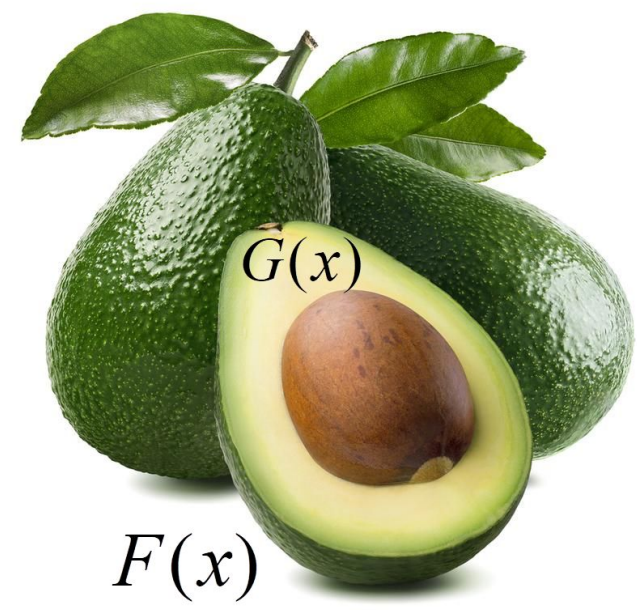

Fig. 3: The core $G(x)$ is a convex closed subset of $F(x)$.

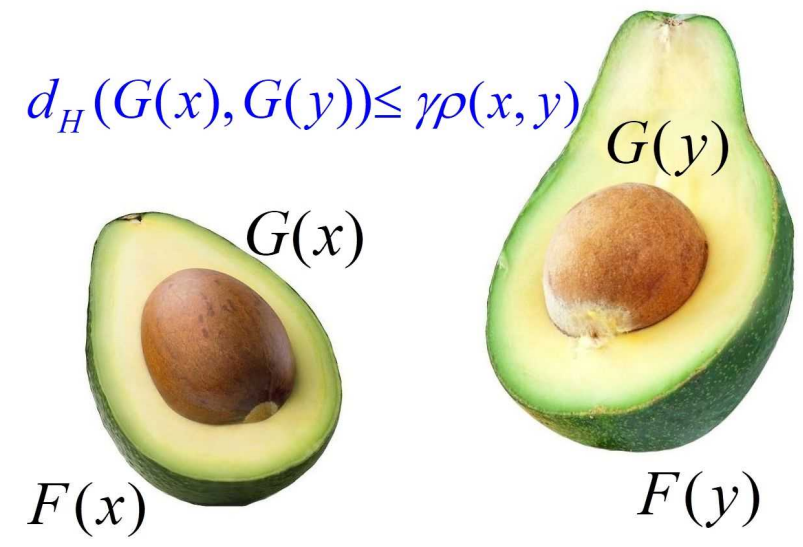

Fig. 4: The $\gamma$-core $G$ is $\gamma$-Lipschitz with respect to the Hausdorff distance.

Recall that the Hausdorff distance $\mathrm{d}_{\mathrm{H}}(A, B)$ between two non-empty bounded subsets $A$ and $B$ of $X$ is defined by

$$
\mathrm{d}_{\mathrm{H}}(A, B)=\inf \left\{r>0: A+B_{X}(0, r) \supset B \text { and } B+B_{X}(0, r) \supset A\right\} .
$$

Here and throughout this paper, for each $x \in X$ and $r>0$, we use the standard notation $B_{X}(x, r)$ for the closed ball in $X$ with center $x$ and radius $r$. We also let $B_{X}=B_{X}(0,1)$ denote the unit ball in $X$, and we write $r B_{X}$ to denote the ball $B_{X}(0, r)$.

In Definition $1.3 \mathrm{~m}$ can be any non-negative integer not exceeding the dimension of the Banach space $X$. It can happen that a core $G: \mathcal{M} \rightarrow \mathcal{K}_{m}(X)$ of a given set-valued mapping $F: \mathcal{M} \rightarrow \mathcal{K}_{m}(X)$ in fact maps $\mathcal{M}$ into the smaller collection $\mathcal{K}_{m^{\prime}}(X)$ for some integer $m^{\prime} \in[0, m)$. The next claim shows that the existence of some core $G: \mathcal{M} \rightarrow$ $\mathcal{K}_{m}(X)$ for $F$ implies the existence of a (possibly different) core which maps $\mathcal{M}$ into $\mathcal{K}_{0}(X)$. Since $\mathcal{K}_{0}(X)$ is identified with $X$, that core is simply a Lipschitz selection of $F$. 
Claim 1.4. ( [16] Section 5]) Let $\gamma$ be a positive constant, let $m$ be a non-negative integer, and let $G: \mathcal{M} \rightarrow \mathcal{K}_{m}(X)$ be a $\gamma$-core of a set-valued mapping $F: \mathcal{M} \rightarrow \mathcal{K}_{m}(X)$ for some Banach space $X$. Then $F$ has a Lipschitz selection $f: \mathcal{M} \rightarrow X$ with $\|f\|_{\operatorname{Lip}(\mathcal{M}, X)} \leq C \gamma$ where $C=C(m)$ is a constant depending only on $m$.

In [16] we showed that this claim follows from Definition 1.3 and the existence of the so-called "Steiner-type point" map St : $\mathcal{K}_{m}(X) \rightarrow X$ [25]. See formula (1.12).

In [16] given a set-valued mapping $F: \mathcal{M} \rightarrow \mathcal{K}_{m}(X)$ satisfying the hypothesis of Theorem 1.2, we constructed a $\gamma$-core $G$ of $F$ with a positive constant $\gamma$ depending only on $m$. We produced the core $G$ using a rather delicate and complicated procedure whose main ingredients are families of Basic Convex Sets associated with $F$, metric spaces with bounded Nagata dimension, ideas and methods of work [14] related to the case $\mathcal{M}=\mathbf{R}^{n}$, and Lipschitz selections on finite metric trees. See [16] for more details.

In the present paper we suggest and discuss a different new geometrical method for producing a core of a set-valued mapping. Its main ingredient is the so-called balanced refinement of a set-valued mapping which we define as follows.

Definition 1.5. Let $\lambda \geq 0$, let $(\mathcal{M}, \rho)$ be a pseudometric space, let $X$ be a Banach space, and let $F: \mathcal{M} \rightarrow \mathcal{K}_{m}(X)$ be a set-valued mapping for some non-negative integer $m$. For each $x \in \mathcal{M}$ we consider the subset of $F(x)$ defined by

$$
\mathcal{B R}[F: \lambda ; \rho](x)=\bigcap_{z \in \mathcal{M}}\left[F(z)+\lambda \rho(x, z) B_{X}\right] .
$$

We refer to the set-valued mapping $\mathcal{B R}[F: \lambda ; \rho]: \mathcal{M} \rightarrow \mathcal{K}_{m}(X) \cup\{\emptyset\}$ as the $\lambda$-balanced refinement of the mapping $F$.

We note that any Lipschitz selection $f$ of a set-valued mapping $F: \mathcal{M} \rightarrow \mathcal{K}_{m}(X)$ with $\|f\|_{\operatorname{Lip}(\mathcal{M}, X)} \leq \lambda$ is also a Lipschitz selection of the $\lambda$-balanced refinement of $F$, i.e.,

$$
f(x) \in \mathcal{B R}[F: \lambda ; \rho](x) \text { for all } \quad x \in \mathcal{M} .
$$

Various geometrical parameters of the set $\mathcal{B R}[F: \lambda ; \rho](x)$ (such as diameter and width, etc.) may turn out to be smaller than the same parameters for the set $F(x)$ which contains it. When attempting to find Lipschitz selections of $F$ it may turn out to be convenient for our purposes to search for them in the more "concentrated" setting provided by the sets $\mathcal{B R}[F: \lambda ; \rho](x)$. One can take this approach still further by searching in even smaller sets which can be obtained from consecutive iterations of balanced refinements of $F$, i.e., the sets which we describe in the following definition.

Definition 1.6. Let $\ell$ be a positive integer, and let $\vec{\lambda}=\left\{\lambda_{k}: 1 \leq k \leq \ell\right\}$ be a finite sequence of $\ell$ non-negative numbers $\lambda_{k}$. We set $F^{[0]}=F$, and, for every $x \in \mathcal{M}$ and integer $k \in[0, \ell-1]$, we define

$$
F^{[k+1]}(x)=\mathcal{B R}\left[F^{[k]}: \lambda_{k+1} ; \rho\right](x)=\bigcap_{z \in \mathcal{M}}\left[F^{[k]}(z)+\lambda_{k+1} \rho(x, z) B_{X}\right] .
$$

We refer to the set-valued mapping $F^{[k]}: \mathcal{M} \rightarrow \mathcal{K}_{m}(X) \cup\{\emptyset\}, k \in[1, \ell]$, as the $k$-th order $(\vec{\lambda}, \rho)$-balanced refinement of $F$. 
Clearly,

$$
F^{[k+1]}(x) \subset F^{[k]}(x) \quad \text { on } \mathcal{M} \text { for every } k \in[0, \ell-1]
$$

(Put $z=x$ in the right hand side of $(1.3)$.

Remark 1.7. Of course, for each integer $k \in[1, \ell]$ the set $F^{[k]}(x)$ also depends on the sequence $\vec{\lambda}=\left\{\lambda_{k}: 1 \leq k \leq \ell\right\}$, on the pseudometric space $\mathfrak{M}=(\mathcal{M}, \rho)$ and the Banach space $X$. However, in all places where we use $F^{[k]}$ 's, these objects, i.e., $\vec{\lambda}, \mathfrak{M}$ and $X$, are clear from the context. Therefore, in these cases, we omit any mention of $\vec{\lambda}, \mathfrak{M}$ and $X$ in the notation of $F^{[k]}$ 's. $\triangleleft$

We formulate the following

Conjecture 1.8. Let $(\mathcal{M}, \rho)$ be a pseudometric space, and let $X$ be a Banach space. Let $m$ be a fixed positive integer and (as in the formula (1.1) of Theorem 1.2) let $N(m, X)$ denote the "finiteness number" $N(m, X)=2^{\ell}$ where $\ell=\ell(m, X)=\min \{m+1, \operatorname{dim} X\}$.

There exist a constant $\gamma \geq 1$ and a sequence $\vec{\lambda}=\left\{\lambda_{k}: 1 \leq k \leq \ell\right\}$ of $\ell$ numbers $\lambda_{k}$ all satisfying $\lambda_{k} \geq 1$ such that the following holds:

Let $F: \mathcal{M} \rightarrow \mathcal{K}_{m}(X)$ be a set-valued mapping such that, for every $\mathcal{M}^{\prime} \subset \mathcal{M}$ with $\# \mathcal{M}^{\prime} \leq N(m, X)$, the restriction $\left.F\right|_{\mathcal{M}^{\prime}}$ of $F$ to $\mathcal{M}^{\prime}$ has a Lipschitz selection $f_{\mathcal{M}^{\prime}}: \mathcal{M}^{\prime} \rightarrow X$ with Lipschitz seminorm $\left\|f_{\mathcal{M}^{\prime}}\right\|_{\operatorname{Lip}\left(\mathcal{M}^{\prime}, X\right)} \leq 1$.

Then the $\ell$-th order balanced refinement of the mapping $F$, namely the set-valued mapping $F^{[\ell]}: \mathcal{M} \rightarrow \mathcal{K}_{m}(X)$ is a $\gamma$-core of $F$.

Here $F^{[\ell]}$ is defined as in Definition 1.6 using the particular sequence $\vec{\lambda}$.

Our main results, Theorem 1.9 and Theorem 1.10 below, state that Conjecture 1.8 holds in two special cases, when either (i) $m=2$ and $\operatorname{dim} X=2$, or (ii) $m=1$ and $X$ is an arbitrary Banach space. Note that in both of these cases the above mentioned finiteness number $N(m, X)$ equals 4 .

Theorem 1.9. Let $\mathfrak{M}=(\mathcal{M}, \rho)$ be a pseudometric space, and let $X$ be a two dimensional Banach space. Let $m=2$ so that the number $\ell(m, X)=2$. In this case Conjecture 1.8 holds for every $\lambda_{1}, \lambda_{2}$ and $\gamma$ such that

$$
\lambda_{1} \geq e(\mathfrak{M}, X), \quad \lambda_{2} \geq 3 \lambda_{1}, \quad \gamma \geq \lambda_{2}\left(3 \lambda_{2}+\lambda_{1}\right)^{2} /\left(\lambda_{2}-\lambda_{1}\right)^{2} .
$$

Here e $(\mathfrak{M}, X)$ denotes the Lipschitz extension constant of $X$ with respect to $\mathfrak{M}$. (See Definition 3.1)

Thus, the following statement is true: Let $F: \mathcal{M} \rightarrow \mathcal{K}(X)$ be a set-valued mapping from a pseudometric space $(\mathcal{M}, \rho)$ into the family $\mathcal{K}(X)$ of all non-empty convex compact subsets of $X$. Given $x \in \mathcal{M}$ let

$$
F^{[1]}(x)=\bigcap_{z \in \mathcal{M}}\left[F(z)+\lambda_{1} \rho(x, z) B_{X}\right], \quad F^{[2]}(x)=\bigcap_{z \in \mathcal{M}}\left[F^{[1]}(z)+\lambda_{2} \rho(x, z) B_{X}\right] .
$$

Suppose that for every subset $\mathcal{M}^{\prime} \subset \mathcal{M}$ with $\# \mathcal{M}^{\prime} \leq 4$, the restriction $\left.F\right|_{\mathcal{M}^{\prime}}$ of $F$ to $\mathcal{M}^{\prime}$ has a Lipschitz selection with Lipschitz seminorm at most 1. 
Then for every $\lambda_{1}, \lambda_{2}$ and $\gamma$ satisfying (1.5) the set

$$
F^{[2]}(x) \neq \emptyset \quad \text { for every } \quad x \in \mathcal{M} \text {. }
$$

Furthermore,

$$
\mathrm{d}_{\mathrm{H}}\left(F^{[2]}(x), F^{[2]}(y)\right) \leq \gamma \rho(x, y) \quad \text { for every } \quad x, y \in \mathcal{M} .
$$

If $X$ is a Euclidean two dimensional space, $(1.7)$ and $(1.8)$ hold when $(1.5)$ is replaced by the weaker requirements that

$$
\lambda_{1} \geq e(\mathfrak{M}, X), \quad \lambda_{2} \geq 3 \lambda_{1}, \quad \gamma \geq \lambda_{2}\left\{1+2 \lambda_{2} /\left(\lambda_{2}^{2}-\lambda_{1}^{2}\right)^{\frac{1}{2}}\right\}^{2} .
$$

In particular, in Section 3 we show that the mapping $F^{[2]}$ satisfies $(1.7)$ and $(1.8)$ whenever $X$ is an arbitrary two dimensional Banach space and $\lambda_{1}=4 / 3, \lambda_{2}=4, \gamma=100$. If $X$ is also Euclidean, then one can set $\lambda_{1}=4 / \pi, \lambda_{2}=12 / \pi$ and $\gamma=38$. Furthermore, we prove that if $\mathcal{M}$ is a subset of a Euclidean space $E, \rho$ is the Euclidean metric in $E$, and $X$ is a two dimensional Euclidean space, then properties (1.7) and (1.8) hold for $\lambda_{1}=1$, $\lambda_{2}=3$, and $\gamma=25$.

In Section 5 we prove Theorem 5.5 which refines the result of Theorem 1.9 for the space $X=\ell_{\infty}^{2}$, i.e., for $\mathbf{R}^{2}$ equipped with the norm $\|x\|=\max \left\{\left|x_{1}\right|,\left|x_{2}\right|\right\}, x=\left(x_{1}, x_{2}\right)$. More specifically, we show that in this case properties (1.7) and (1.8) hold whenever $\lambda_{1} \geq 1, \lambda_{2} \geq 3 \lambda_{1}$, and $\gamma \geq \lambda_{2}\left(3 \lambda_{2}+\lambda_{1}\right) /\left(\lambda_{2}-\lambda_{1}\right)$. In particular, these properties hold for $\lambda_{1}=1, \lambda_{2}=3$ and $\gamma=15$.

Let us now explicitly formulate the above mentioned second main result of the paper. We prove it in Section 4. It deals with set-valued mappings from a pseudometric space into the family $\mathcal{K}_{1}(X)$ of all bounded closed line segments of an arbitrary Banach space $X$.

Theorem 1.10. Let $(\mathcal{M}, \rho)$ be a pseudometric space. Let $m=1$ and let $X$ be a Banach space with $\operatorname{dim} X>1$; thus, $\ell(m, X)=2$, see $(1.1)$. In this case Conjecture 1.8 holds for every $\lambda_{1}, \lambda_{2}$ and $\gamma$ such that

$$
\lambda_{1} \geq 1, \quad \lambda_{2} \geq 3 \lambda_{1}, \quad \gamma \geq \lambda_{2}\left(3 \lambda_{2}+\lambda_{1}\right) /\left(\lambda_{2}-\lambda_{1}\right) .
$$

Thus, the following statement is true: Let $F: \mathcal{M} \rightarrow \mathcal{K}_{1}(X)$ be a set-valued mapping such that for every subset $\mathcal{M}^{\prime} \subset \mathcal{M}$ with $\# \mathcal{M}^{\prime} \leq 4$, the restriction $\left.F\right|_{\mathcal{M}^{\prime}}$ of $F$ to $\mathcal{M}^{\prime}$ has a Lipschitz selection with Lipschitz seminorm at most 1.

Let $F^{[2]}$ be the mapping defined by $(1.6)$. Then properties $(1.7)$ and $(1.8)$ hold whenever $\lambda_{1}, \lambda_{2}$ and $\gamma$ satisfy (1.10). In particular, one can set $\lambda_{1}=1, \lambda_{2}=3$ and $\gamma=15$.

If $X$ is a Euclidean space, the same statement is also true whenever, instead of (1.10), $\lambda_{1}, \lambda_{2}$ and $\gamma$ satisfy the weaker condition

$$
\lambda_{1} \geq 1, \quad \lambda_{2} \geq 3 \lambda_{1}, \quad \gamma \geq \lambda_{2}+2 \lambda_{2}^{2} /\left(\lambda_{2}^{2}-\lambda_{1}^{2}\right)^{\frac{1}{2}} .
$$

In particular, in this case, (1.7) and (1.8) hold whenever $\lambda_{1}=1, \lambda_{2}=3$ and $\gamma=10$. 
In Section 5.1 we note that Conjecture 1.8 also holds for a one dimensional space $X$ and $m=1$. In this case the statement of the conjecture is true for every $\lambda_{1} \geq 1$ and $\gamma \geq 1$. See Proposition 5.1 .

Note that Theorem 1.9 tells us that for every set-valued mapping $F$ satisfying the hypothesis of this theorem, the mapping $F^{[2]}$ determined by 1.6 with $\lambda_{1}=4 / 3$ and $\lambda_{2}=4$ provides a $\gamma$-core of $F$ with $\gamma=100$. (See Definition 1.3.) In turn, Theorem 1.10 states that the mapping $F^{[2]}$ corresponding to the parameters $\lambda_{1}=1$ and $\lambda_{2}=3$ is a 15 -core of any $F$ satisfying the conditions of this theorem.

We note that the proofs of Theorem 1.9 and Theorem 1.10 rely on Helly's Intersection Theorem and a series of auxiliary results about neighborhoods of intersections of convex sets. See Section 2.

Remark 1.11. Let us compare Conjecture 1.8 (and Theorems 1.9 and 1.10) with the Finiteness Principle (FP) formulated in Theorem 1.2. First we note that FP is invariant with respect to the transition to an equivalent norm on $X$, while the statement of Conjecture 1.8 is not.

To express this more precisely, let $\|\cdot\|_{1}$ and $\|\cdot\|_{2}$ be two equivalent norms on $X$, i.e., suppose that for some $\alpha \geq 1$ the following inequality $(1 / \alpha)\|\cdot\|_{1} \leq\|\cdot\|_{2} \leq \alpha\|\cdot\|_{1}$ holds. Clearly, if FP holds for $\left(X,\|\cdot\|_{1}\right)$ then it immediately holds also for $\left(X,\|\cdot\|_{2}\right)$ (with the constant $\alpha^{2} \gamma$ instead of $\gamma$ ). However the validity of Conjecture 1.8 for the norm $\|\cdot\|_{1}$ does not imply its validity for an equivalent norm $\|\cdot\|_{2}$ on $X$ (at least we do not see any obvious way for obtaining such an implication). For example, the validity of Conjecture 1.8 in $\ell_{\infty}^{n}$ (i.e., $\mathbf{R}^{n}$ equipped with the uniform norm) does not automatically imply its validity in the space $\ell_{2}^{n}$ (i.e., $\mathbf{R}^{n}$ with the Euclidean norm).

We also note the following: in a certain sense, the result of Theorem 1.9 is "stronger" than Theorem 1.2 (i.e., FP for the case of a two dimensional Banach space $X$ ). Indeed, in this case, the hypotheses of FP and Theorem 1.9 coincide. Moreover, Theorem 1.9 ensures that the set-valued mapping $F^{[2]}$ is a core of $F$. This property of $F^{[2]}$ implies, via arguments in [16] that the function

$$
f(x)=\operatorname{St}\left(F^{[2]}\right)(x), \quad x \in \mathcal{M},
$$

is a Lipschitz selection of $F$. Here St: $\mathcal{K}_{m}(X) \rightarrow X$ is the Steiner-type point map [25].

Thus, FP (in the two dimensional case) follows immediately from Theorem 1.9 . However, it is absolutely unclear how the statement of Theorem 1.9 can be deduced from FP. I would like to thank Charles Fefferman who kindly drew my attention to this interesting fact. $\triangleleft$

Let us reformulate Conjecture 1.8 in a way which does not require the use of the notion of a core of a set-valued mapping. We recall that the mapping $F^{[\ell]}: \mathcal{M} \rightarrow \mathcal{K}_{m}(X)$ which appears in Conjecture 1.8 is a $\gamma$-core of $F$ if

$$
\mathrm{d}_{\mathrm{H}}\left(F^{[\ell]}(x), F^{[\ell]}(y)\right) \leq \gamma \rho(x, y) \text { for all } x, y \in \mathcal{M} .
$$

See part (ii) of Definition 1.3. Hence, given $x \in \mathcal{M}$,

$$
F^{[\ell]}(x) \subset F^{[\ell]}(y)+\gamma \rho(x, y) B_{X} \text { for every } y \in \mathcal{M} .
$$


Let

$$
F^{[\ell+1]}(x)=\mathcal{B R}\left[F^{[\ell]}: \gamma ; \rho\right](x)=\bigcap_{y \in \mathcal{M}}\left[F^{[\ell]}(y)+\gamma \rho(x, y) B_{X}\right] .
$$

Cf. 1.3). This and 1.13 imply the inclusion $F^{[\ell+1]}(x) \supset F^{[\ell]}(x), x \in \mathcal{M}$. On the other hand, 1.4 tells us that $F^{[\ell+1]}(x) \subset F^{[\ell]}(x)$ proving that $F^{[\ell+1]}=F^{[\ell]}$ on $\mathcal{M}$.

These observations enable us to reformulate Conjecture 1.8 as follows.

Conjecture 1.12. Let $(\mathcal{M}, \rho)$ be a pseudometric space, and let $X$ be a Banach space. Let $m$ be a fixed positive integer and let $\ell=\ell(m, X)$, see( $(1.1)$.

There exists a sequence $\vec{\lambda}=\left\{\lambda_{k}: 1 \leq k \leq \ell+1\right\}$ of $\ell+1$ numbers $\lambda_{k}$ all satisfying $\lambda_{k} \geq$ 1 such that, for every set-valued mapping $F: \mathcal{M} \rightarrow \mathcal{K}_{m}(X)$ satisfying the hypothesis of the Finiteness Principle (Theorem 1.2), the family $\left\{F^{[k]}: k=1, \ldots, \ell+1\right\}$ of set-valued mappings constructed by formula (1.3) has the following property:

$$
F^{[\ell]}(x) \neq \emptyset \quad \text { and } \quad F^{[\ell+1]}(x)=F^{[\ell]}(x) \text { for all } x \in \mathcal{M} .
$$

We refer to (1.14) as a Stabilization Property of balanced refinements.

Thus, Theorem 1.9 and Theorem 1.10 tell us that a Stabilization Property of balanced refinements holds whenever $\operatorname{dim} X=2$ or $m=1$ (and $X$ is an arbitrary). More specifically, Theorem 1.9 shows that if $m=2$ and $\operatorname{dim} X=2$, Conjecture 1.12 holds with $\ell=2$ and $\vec{\lambda}=\left\{4 / 3,4,10^{2}\right\}$.

In other words, in this case, $F^{[2]}(x) \neq \emptyset$ for each $x \in \mathcal{M}$ and $F^{[3]}=F^{[2]}$ on $\mathcal{M}$. In turn, Theorem 1.10 states that the same property holds whenever $X$ is an arbitrary Banach space, $m=1$, and $\vec{\lambda}=\{1,3,15\}$.

Acknowledgements. I am very thankful to Michael Cwikel for useful suggestions and remarks. I am also very grateful to Charles Fefferman for stimulating discussions and valuable advice.

The results of this paper were presented at the 12th Whitney Problems Workshop, August 2019, the University of Texas at Austin, TX. I am very thankful to all participants of that workshop for valuable conversations and useful remarks. I am also grateful to the Conference Board of the Mathematical Sciences and the University of Texas for supporting the Austin workshop.

\section{Neighborhoods of intersections of convex sets in a Banach space.}

We first need to fix some notation.

Let $(X,\|\cdot\|)$ be a Banach space, and let $B_{X}$ be the unit ball in $X$. Let $A$ and $B$ be non-empty subsets of $X$. We let $A+B=\{a+b: a \in A, b \in B\}$ denote the Minkowski sum of these sets. By $\mathbb{C}(X)$ we denote the family of all convex closed non-empty subsets of $X$. We equip this family with the Hausdorff distance.

Sometimes, for a given set $\mathcal{M}$, we will be looking simultaneously at two distinct pseudometrics on $\mathcal{M}$, say $\rho$ and $\delta$. In this case we will speak of a $\rho$-Lipschitz selection and $\rho$-Lipschitz seminorm, or a $\delta$-Lipschitz selection and $\delta$-Lipschitz seminorm to make clear which pseudometric we are using. Furthermore, given a mapping $f: \mathcal{M} \rightarrow X$ 
we will write $\|f\|_{\operatorname{Lip}((\mathcal{M} ; \rho), X)}$ or $\|f\|_{\operatorname{Lip}((\mathcal{M} ; \delta), X)}$ to denote the Lipschitz seminorm of $f$ with respect to the pseudometric $\rho$ or $\delta$ respectively.

For each finite set $S$, we let $\# S$ denote the number of elements of $S$.

Given a Banach space $X$, Przesławski and Yost [21] introduced an important geometrical characteristic of $X$, the so-called modulus of squareness of $X$. Let us recall its definition.

We observe that for any $x, y \in X$ with $\|y\|<1<\|x\|$ there exists a unique $z=z(x, y)$ with $\|z\|=1$ which belongs to the line segment $[x, y]$. We set

$$
\omega(x, y)=\frac{\|x-z(x, y)\|}{\|x\|-1}
$$

and define a function $\xi:[0,1) \rightarrow[1, \infty)$ by

$$
\xi_{X}(\beta)=\sup \{\omega(x, y): x, y \in X,\|y\| \leq \beta<1<\|x\|\} .
$$

We also put

$$
\varphi(\beta)=(1+\beta) /(1-\beta) \quad \text { and } \quad \psi(\beta)=\left(1-\beta^{2}\right)^{-\frac{1}{2}}, \quad \beta \in[0,1) .
$$

It is shown in [21], that for any Banach space $X$

$$
\xi_{X}(\beta) \leq \varphi(\beta) \text { for every } \beta \in[0,1),
$$

and

$$
\xi_{X}(\beta)=\psi(\beta) \text { for every } \beta \in[0,1),
$$

provided $X$ is a Euclidean space.

Theorem 2.1. ( [21] Theorem 4]) Let $(S, \delta)$ be a metric space, let $X$ be a Banach space, and let $f: S \rightarrow X$ and $g: S \rightarrow[0, \infty)$ be Lipschitz mappings. Let $F: S \rightarrow \mathfrak{C}(X)$ be a Lipschitz (with respect to the Hausdorff distance) set-valued mapping.

Suppose that there exists a constant $\gamma>1$ such that $g(x) \geq \gamma \operatorname{dist}(f(x), F(x))$ for every $x \in S$. Then the intersection mapping $G: S \rightarrow \mathfrak{C}(X)$ defined by

$$
G(x)=F(x) \cap B_{X}(f(x), g(x))
$$

is Lipschitz continuous on $S$ (with respect to $\mathrm{d}_{\mathrm{H}}$ ) with Lipschitz seminorm

$$
\|G\|_{\operatorname{Lip}(S, \mathfrak{E}(X))} \leq\|F\|_{\operatorname{Lip}(S, \mathfrak{E}(X))}+\left(\|F\|_{\operatorname{Lip}(S, \mathbb{E}(X))}+\|f\|_{\operatorname{Lip}(S, X)}+\|g\|_{\operatorname{Lip}(S, \mathbf{R})}\right) \xi(1 / \gamma) .
$$

This theorem implies the following

Proposition 2.2. Let $X$ be a Banach space, $a \in X, r \geq 0$, and let $C \subset X$ be a convex set.

Suppose that $C \cap B_{X}(a, r) \neq \emptyset$. Then for every $s>0$ and every $L>1$ the following inequality

$$
\mathrm{d}_{\mathrm{H}}\left(C \cap B_{X}(a, L r),\left(C+s B_{X}\right) \cap B_{X}(a, L r+s)\right) \leq\left(1+2 \xi_{X}\left(\frac{1}{L}\right)\right) s
$$

holds. 
Proof. Let $S=\{x, y\} \subset \mathbf{R}$ where $x=0$ and $y=s$, and let $\delta(x, y)=s$.

We define a mapping $f: S \rightarrow X$ and a function $g: S \rightarrow \mathbf{R}$ by letting $f(x)=f(y)=a$ and $g(x)=L r, g(y)=L r+s$. Clearly, $\|f\|_{\operatorname{Lip}(S, X)}=0$, and $\|g\|_{\operatorname{Lip}(S, \mathbf{R})}=1$.

We put $\gamma=L$. We note that $C \cap B_{X}(a, r) \neq \emptyset$ so that

$$
\operatorname{dist}(f(x), F(x))=\operatorname{dist}(a, C) \leq r .
$$

Hence, $g(x)=L r=\gamma r \geq \gamma \operatorname{dist}(f(x), F(x))$.

Then we define a mapping $F: S \rightarrow \mathfrak{C}(X)$ by setting $F(x)=C$ and $F(y)=C+s B_{X}$. Clearly,

$$
\mathrm{d}_{\mathrm{H}}(F(x), F(y)) \leq s=\delta(x, y) \quad \text { so that } \quad\|F\|_{\operatorname{Lip}(S, \mathbb{C}(X))} \leq 1 .
$$

Thus, the conditions of Theorem 2.1 are satisfied for the metric space $(S, \delta)$ and the mappings $f, g$ and $F$. This theorem tells us that the mapping $G: S \rightarrow \mathfrak{C}(X)$ defined by

$$
G(u)=F(u) \cap B_{X}(f(u), g(u)), \quad u \in S,
$$

is Lipschitz on $S$ with respect to the Hausdorff distance. Furthermore,

$\|G\|_{\operatorname{Lip}(S, \mathbb{C}(X))} \leq\|F\|_{\operatorname{Lip}(S, \mathfrak{C}(X))}+\left(\|F\|_{\operatorname{Lip}(S, \mathbb{E}(X))}+\|f\|_{\operatorname{Lip}(S, X)}+\|g\|_{\operatorname{Lip}(S, \mathbf{R})}\right) \xi(1 / \gamma) \leq 1+2 \xi_{X}\left(\frac{1}{L}\right)$.

Hence,

$$
\begin{aligned}
\mathrm{d}_{\mathrm{H}}\left(C \cap B_{X}(a, L r),\left(C+s B_{X}\right) \cap B_{X}(a, L r+s)\right) & =\mathrm{d}_{\mathrm{H}}(G(x), G(y)) \\
& \leq\|G\|_{\operatorname{Lip}(S, \mathbb{E}(X))} \delta(x, y) \leq\left(1+2 \xi_{X}\left(\frac{1}{L}\right)\right) s
\end{aligned}
$$

proving the proposition.

Proposition 2.2 implies the following important

Theorem 2.3. Let $X$ be a Banach space, and let $C \subset X$ be a convex set. Let $a \in X$ and let $r \geq 0$. Suppose that

$$
C \cap B_{X}(a, r) \neq \emptyset
$$

Then for every $s>0$ and $L>1$

$$
\left[C \cap B_{X}(a, L r)\right]+\theta(L) s B_{X} \supset\left(C+s B_{X}\right) \cap B_{X}(a, L r+s)
$$

where

$$
\theta(L)=(3 L+1) /(L-1) .
$$

If $X$ is a Euclidean space then (2.7) holds with

$$
\theta(L)=1+\frac{2 L}{\sqrt{L^{2}-1}} .
$$


Proof. Let

$$
G=C \cap B_{X}(a, L r) \text { and } \widetilde{G}=\left(C+s B_{X}\right) \cap B_{X}(a, L r+s) .
$$

Definition 1.2 tells us that $\widetilde{G} \subset G+\mathrm{d}_{\mathrm{H}}(G, \widetilde{G}) B_{X}$. In turn, Proposition 2.2 states that

$$
\mathrm{d}_{\mathrm{H}}(G, \widetilde{G}) \leq\left(1+2 \xi_{X}\left(\frac{1}{L}\right)\right) s .
$$

Hence,

$$
\widetilde{G} \subset G+\Theta(L) s B_{X} \quad \text { where } \quad \Theta(L)=1+2 \xi_{X}\left(\frac{1}{L}\right) .
$$

Now, let $X$ be an arbitrary Banach space. In this case, thanks to (2.3) and (2.4), we have

$$
\Theta(L)=1+2 \xi_{X}\left(\frac{1}{L}\right) \leq 1+2 \frac{1+1 / L}{1-1 / L}=\frac{3 L+1}{L-1} .
$$

This inequality and (2.8) imply inclusion (2.7) in the case under consideration.

Finally, let $X$ be a Euclidean space. In this case, from (2.3), (2.5) and (2.9), we have

$$
\Theta(L)=1+2 \xi_{X}\left(\frac{1}{L}\right)=1+2\left(1-(1 / L)^{2}\right)^{-\frac{1}{2}}=1+\frac{2 L}{\sqrt{L^{2}-1}}=\theta(L)
$$

The proof of the theorem is complete.

For the case of an arbitrary Banach space $X$, Theorem 2.3 was proved by Przesławski and Rybinski [19, p. 279]. For the case of a Euclidean space $X$ see Przesławski, Yost [21, Theorem 4]. For similar results we refer the reader to [1], [2, p. 369] and [4, p. 26].

For the sake of completeness, and for the reader's convenience, below we give

A direct proof of Theorem 2.3. We follow the proof of Lemma 5.3 from [19, p. 279]. If $r=0$ then 2.7) holds trivially, so we assume that $r>0$. Without loss of generality, we may also assume that $a=0$. Thus we should prove that

$$
\left[C \cap\left(L r B_{X}\right)\right]+\theta s B_{X} \supset\left(C+s B_{X}\right) \cap\left(L r B_{X}+s B_{X}\right)
$$

provided $r>0, s>0, L>1$. Let

$$
z \in\left(C+s B_{X}\right) \cap\left(L r B_{X}+s B_{X}\right)=\left(C+s B_{X}\right) \cap\left[(L r+s) B_{X}\right] .
$$

Prove that

$$
z \in\left[C \cap\left(\operatorname{Lr} B_{X}\right)\right]+\theta s B_{X}
$$

Thanks to 2.10), $z \in\left(C+s B_{X}\right)$ so that there exists an element $v \in C$ such that

$$
\|v-z\| \leq s .
$$

If $\|v\| \leq L r$, then $v \in C \cap\left(\operatorname{Lr} B_{X}\right)$ proving 2.11).

Suppose that

$$
\|v\|>\operatorname{Lr} .
$$


Property (2.10) tells us that $\|z\| \leq L r+s$ so that

$$
\|v\| \leq\|z\|+s \leq L r+2 s .
$$

In turn, assumption (2.6) tells us that there exists an element $v^{\prime} \in C$ such that

$$
\left\|v^{\prime}\right\| \leq r<L r .
$$

Choose $\lambda \in(0,1)$ such that the element

$$
\tilde{v}=\lambda v^{\prime}+(1-\lambda) v
$$

has the norm $\|\tilde{v}\|=L r$. See Fig. 5 .

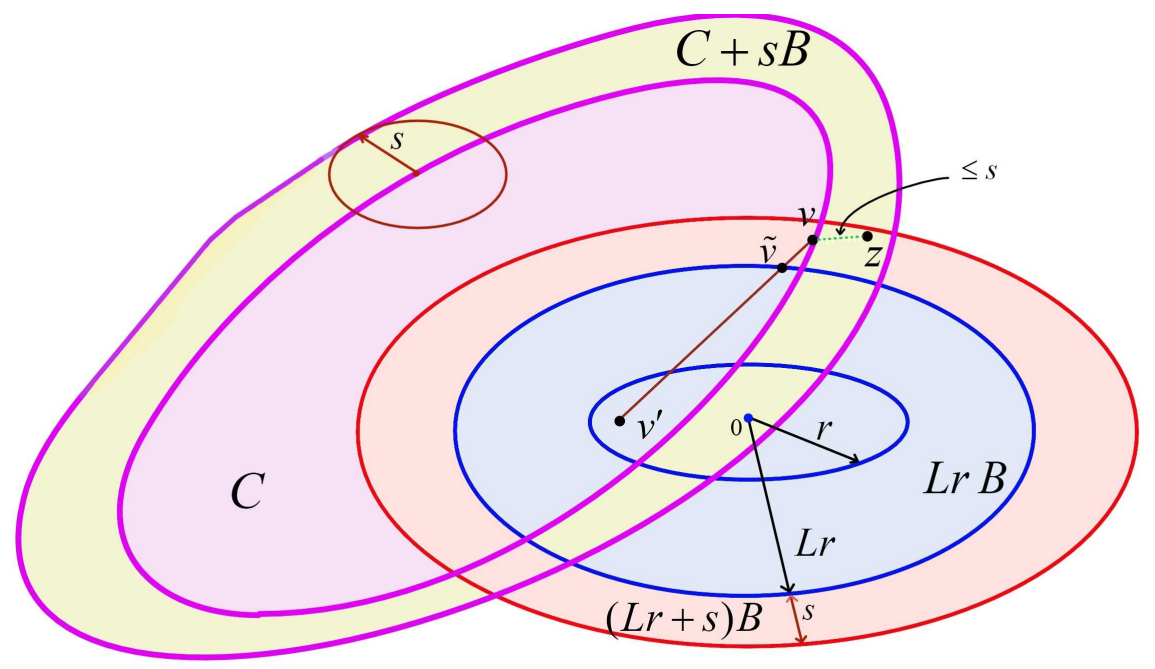

Fig. 5: The points $z, v, \tilde{v}$ and $v^{\prime}$.

We know that $C$ is convex so that $\left[v^{\prime}, v\right] \subset C$ proving that

$$
\tilde{v} \in C \cap\left(\operatorname{Lr} B_{X}\right)
$$

Thanks to (2.15), (2.14) and the triangle inequality,

$$
L r=\|\tilde{v}\|=\left\|\lambda v^{\prime}+(1-\lambda) v\right\| \leq \lambda r+(1-\lambda)(L r+2 s)
$$

proving that

$$
\lambda \leq \frac{2 s}{(L-1) r+2 s} .
$$

Consequently, thanks to this inequality, (2.15) and (2.14)

$\|v-\tilde{v}\|=\lambda\left\|v-v^{\prime}\right\| \leq \lambda\left(\|v\|+\left\|v^{\prime}\right\|\right) \leq \frac{2 s}{((L-1) r+2 s)} \cdot(L r+2 s+r) \leq 2 s(L+1) /(L-1)$. 
From this inequality and (2.12) we have

$$
\|z-\tilde{v}\| \leq\|z-v\|+\|v-\tilde{v}\| \leq s+2 s(L+1) /(L-1)=\theta(L) s
$$

which together with 2.16) implies 2.11).

Let now $X$ be a Euclidean space. We modify the above proof after $(2.16)$ as follows. We put $\beta=1 / L$, and

$$
x=\frac{1}{L r} v, \quad y=\frac{1}{L r} v^{\prime}, \quad w=\frac{1}{L r} \tilde{v} .
$$

Then, thanks to 2.13) and 2.15,

$$
\|y\| \leq \beta<1<\|x\| .
$$

We note that for any $u, \tilde{u} \in X$ such that $\|\tilde{u}\|<1<\|u\|$, there exists a unique point $w=w(u, \tilde{u}) \in[u, \tilde{u}]$ with $\|w\|=1$. Hence, thanks to (2.1),

$$
\omega(u, \tilde{u})=\frac{\|u-w(u, \tilde{u})\|}{\|u\|-1} . \quad \text { See Fig. } 6 .
$$

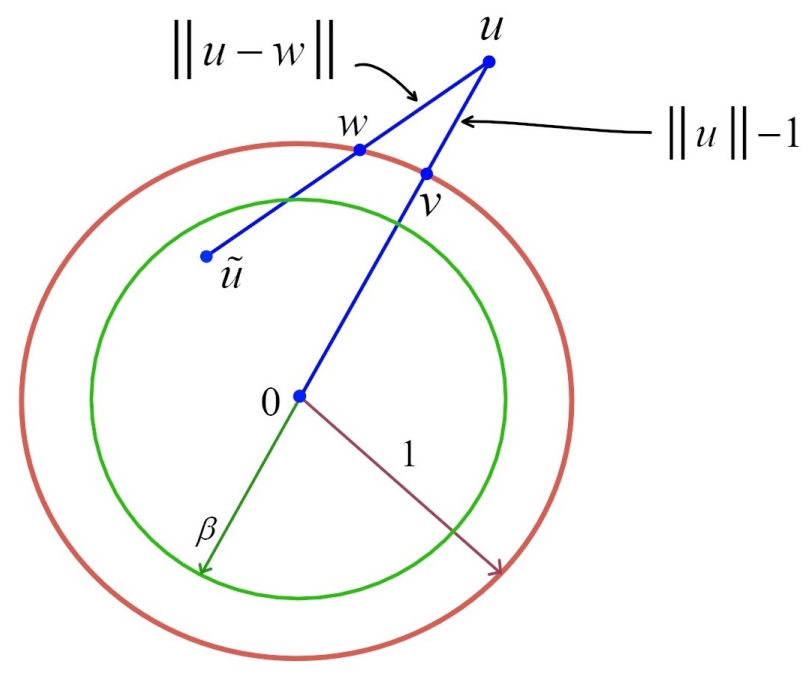

Fig. 6: The points $u, w$ and $\tilde{u}$.

We also recall the definition of the function $\xi_{X}$, see (2.2):

$$
\xi_{X}(\beta)=\sup \{\omega(u, \tilde{u}): u, \tilde{u} \in X,\|\tilde{u}\| \leq \beta<1<\|u\|\} .
$$

Prove that

$$
\xi_{X}(\beta)=\left(1-\beta^{2}\right)^{-\frac{1}{2}}
$$

In fact, fix $u$ with $\|u\|>1$. One can easily see that $\sup \{\omega(u, \tilde{u}):\|\tilde{u}\| \leq \beta\}$ is attained for some $\tilde{u}$ with $\|\tilde{u}\|=\beta$, and the line segment $[\tilde{u}, u]$ touches the sphere centered at the origin with radius $\beta$. Thus, $u-\tilde{u}$ is perpendicular to $\tilde{u}$. See Fig. 7 . 


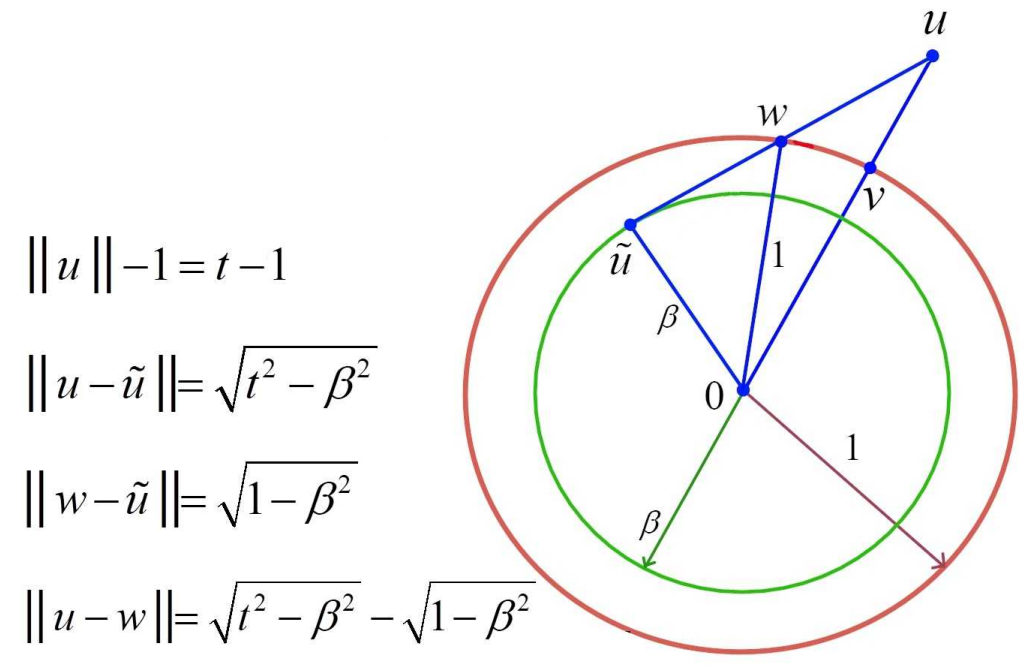

Fig. 7: The optimal position of the point $\tilde{u}$ for given $u$ with $\|u\|=t>1$.

Hence, $\sup \{\omega(u, \tilde{u}):\|\tilde{u}\| \leq \beta\}=f(\|u\|)$ where

$$
f(t)=\frac{\sqrt{t^{2}-\beta^{2}}-\sqrt{1-\beta^{2}}}{t-1}, \quad t>1 .
$$

The function $f$ is decreasing on $(1,+\infty)$ so that

$$
\sup _{t>1} f(t)=\lim _{t \rightarrow 1} f(t)=\left(1-\beta^{2}\right)^{-\frac{1}{2}}
$$

proving (2.21).

We apply formula (2.21) to the points $x, y, w$ defined by (2.17), and to $\beta=1 / L$. We have

$$
\frac{\|v-\tilde{v}\|}{\|v\|-L r}=\frac{\|x-w\|}{\|y\|-1}=\omega(x, y) .
$$

See (2.19). Thanks to (2.18) and (2.20),

$$
\frac{\|v-\tilde{v}\|}{\|v\|-L r} \leq \sup \{\omega(u, \tilde{u}): u, \tilde{u} \in X,\|\tilde{u}\| \leq \beta<1<\|u\|\}=\xi_{X}(\beta)=\xi_{X}(1 / L)
$$

so that, thanks to 2.21,

$$
\frac{\|v-\tilde{v}\|}{\|v\|-L r} \leq \xi_{X}(1 / L)=\frac{L}{\sqrt{L^{2}-1}} .
$$

In turn, thanks to (2.14), $\|v\|-L r \leq 2 s$, so that

$$
\|v-\tilde{v}\| \leq \frac{L}{\sqrt{L^{2}-1}}(\|v\|-L r) \leq \frac{2 s L}{\sqrt{L^{2}-1}} .
$$

This inequality and (2.12) imply the following:

$$
\|z-\tilde{v}\| \leq\|z-v\|+\|v-\tilde{v}\| \leq s+\frac{2 s L}{\sqrt{L^{2}-1}}=\left(1+2 L / \sqrt{L^{2}-1}\right) s .
$$


This and (2.16) imply (2.11) with $\theta=\theta(L)$ defined by (2.9).

The proof of Theorem 2.3 is complete.

For the case of an arbitrary Banach space $X$, Theorem 2.3 was proved by Przesławski and Rybinski [19, p. 279]. For the case of a Euclidean space $X$ see Przesławski, Yost [21, Theorem 4]. For similar results we refer the reader to [1], [2, p. 369] and [4, p. 26].

Remark 2.4. In general, assumption (2.6) in Theorem 2.3 cannot be omitted. For instance, let $X=\ell_{\infty}^{2}$ and let $L=2$; then $\theta(L)=7$. Let $r \gg s>0, a \in \mathbf{R}^{2}$, and let $C$ be a half-plane shown in Fig. 8. (In Fig. $8-11$ we omit $X$ in the notation of $\ell_{\infty}^{2}$-balls.)

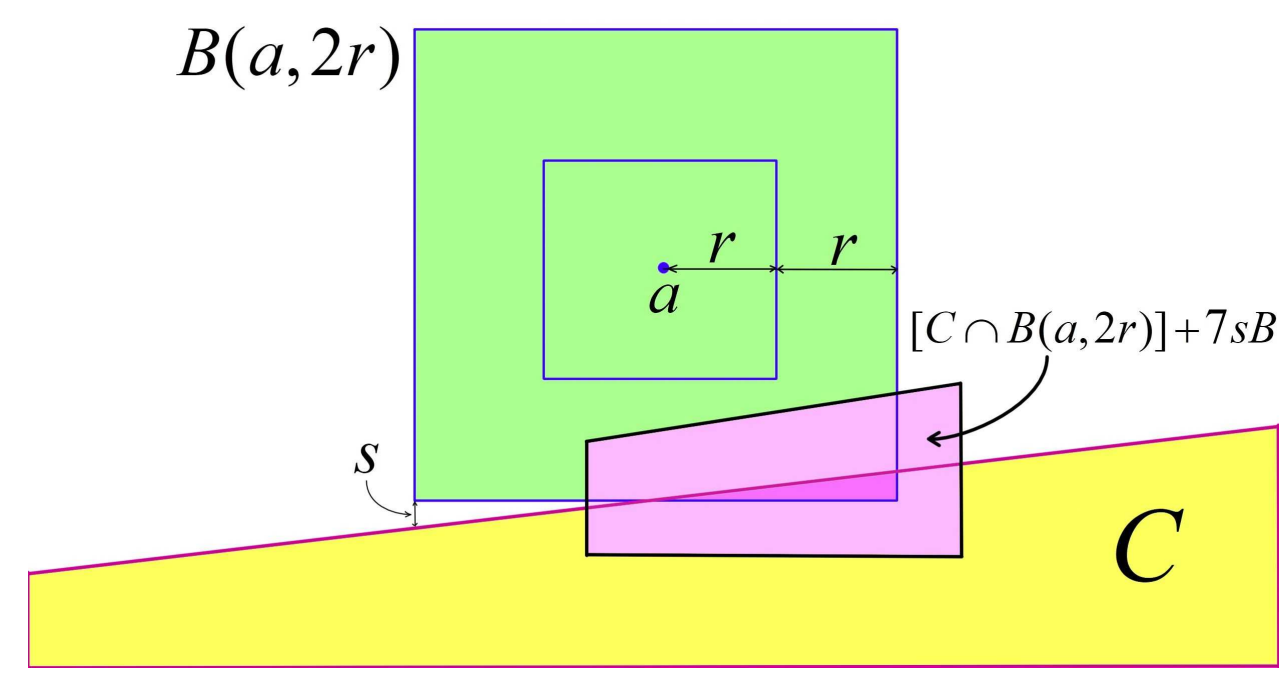

Fig. 8: The half-plane $C$ does not intersect the square $B(a, r)$.

The reader can easily see that in this case imbedding (2.7) does not hold provided $r / s \rightarrow \infty$. Cf. Fig. 8 with Fig. 9 below.

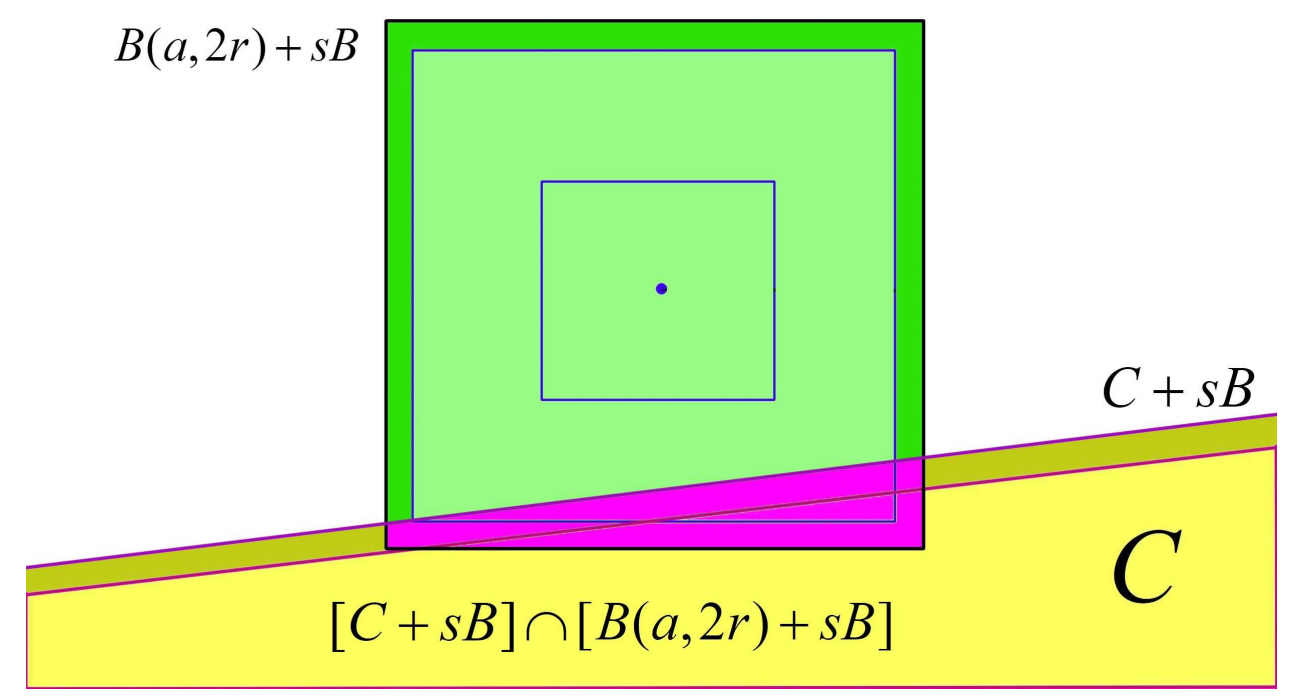

Fig. 9: $\operatorname{diam}\left\{\left[C+s B_{X}\right] \cap[B(a, 2 r)+s B]\right\} \gg \operatorname{diam}\{[C \cap B(a, 2 r)]+7 s B\}$. 
The obvious reason for this is the fact that if condition $(2.6)$ is not met, then the angle between the boundaries of $C$ and $B_{X}(a, 2 r)$ at the point of their intersection is very small.

However, if $B_{X}(a, r) \cap C \neq \emptyset$, than, thanks to 2.7), the sets $A_{1}=C \cap B_{X}(a, 2 r)+7 s B_{X}$ and $A_{2}=\left(C+s B_{X}\right) \cap\left(B_{X}(a, L r)+s B_{X}\right)$ are "compatible", i.e., $A_{1} \supset A_{2}$.

Note that in this case the angle between the boundaries of $C$ and $B_{X}(a, 2 r)$ at the point of their intersection is not small. Cf. Fig. 10 with Fig. 11 below.

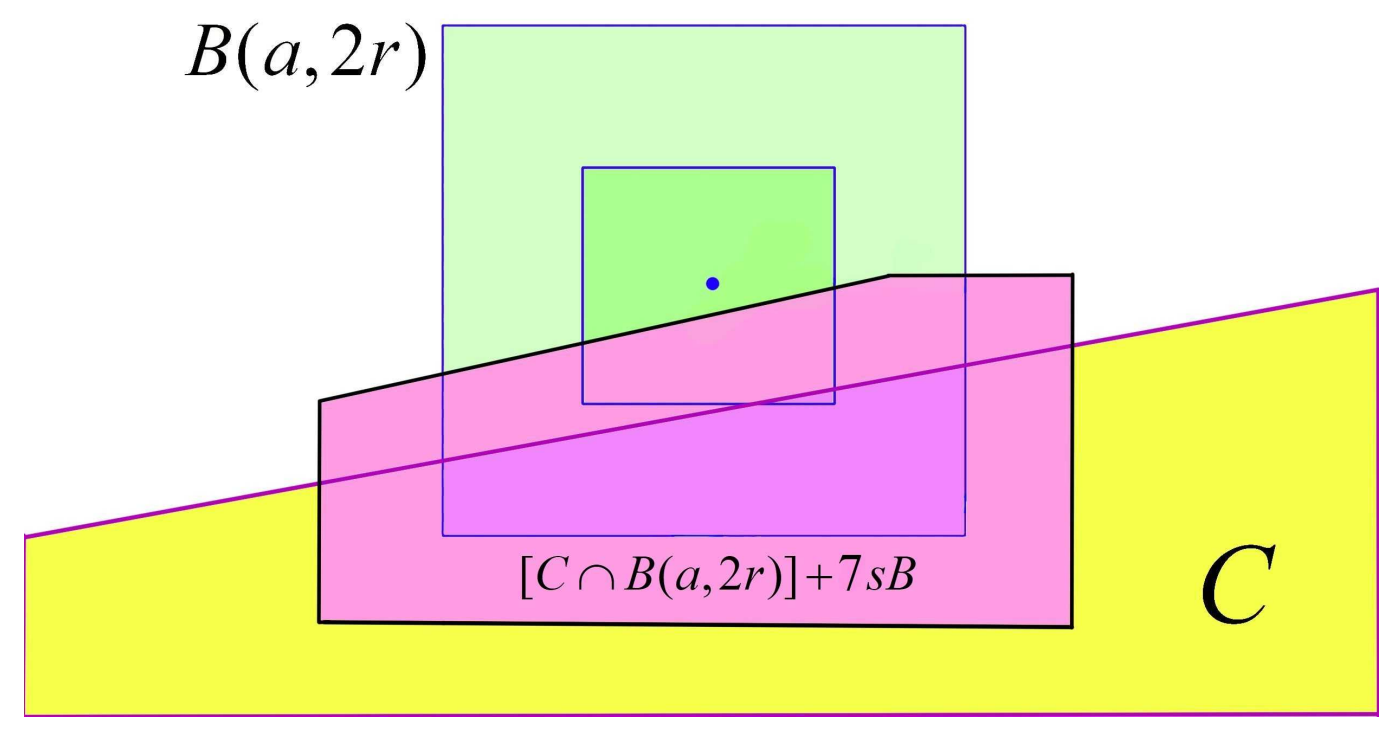

Fig. 10: The set $[C \cap B(a, 2 r)]+7 s B$.

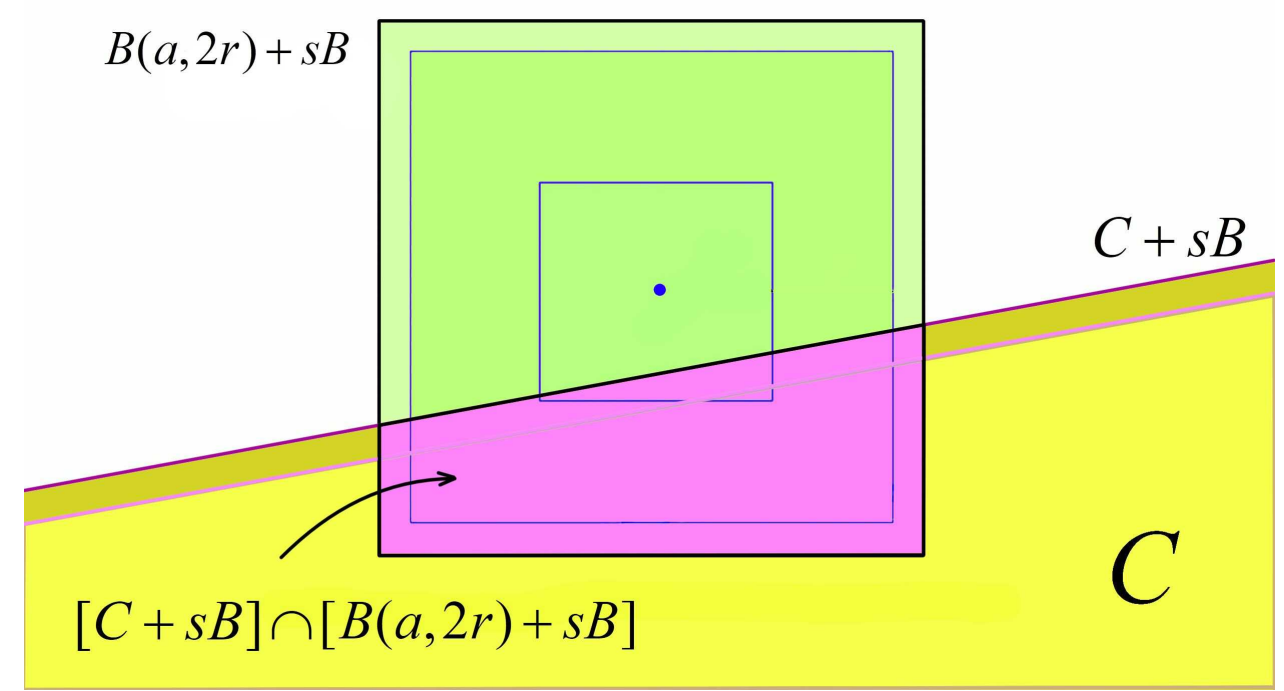

Fig. 11: The set $\left[C+s B_{X}\right] \cap[B(a, 2 r)+s B]$.

Let us recall the classical Helly Intersection Theorem for two dimensional Banach spaces. It can be formulated as follows: 
Theorem 2.5. Let $\mathcal{K}$ be a collection of convex closed subsets of a two dimensional Banach space $X$. Suppose that $\mathcal{K}$ is finite or at least one member of the family $\mathcal{K}$ is bounded.

If every subfamily of $\mathcal{K}$ consisting of at most three elements has a common point then there exists a point common to all of the family $\mathcal{K}$.

We conclude this section by stating and proving one more result which will be one of the main tools for proving our main theorems.

Proposition 2.6. Let $X$ be a two dimensional Banach space. Let $C, C_{1}, C_{2} \subset X$ be convex subsets, and let $r>0$. Suppose that

$$
C_{1} \cap C_{2} \cap\left(C+r B_{X}\right) \neq \emptyset .
$$

Then for every $L>1$ and every $\varepsilon>0$ the following inclusion

$$
\begin{aligned}
& {\left[\left\{\left(C_{1} \cap C_{2}\right)+L r B_{X}\right\} \cap C\right]+\theta(L) \varepsilon B_{X} \supset} \\
& {\left[\left(C_{1} \cap C_{2}\right)+(L r+\varepsilon) B_{X}\right] \cap\left[\left\{\left(C_{1}+r B_{X}\right) \cap C\right\}+\varepsilon B_{X}\right] \cap\left[\left\{\left(C_{2}+r B_{X}\right) \cap C\right\}+\varepsilon B_{X}\right]}
\end{aligned}
$$

holds. The function $\theta$ in the above inclusion is as defined in Theorem 2.3 I.e., for an arbitrary Banach space $X$ that inclusion holds for $\theta(L)=(3 L+1) /(L-1)$, and if $X$ is Euclidean it also holds for $\theta(L)=1+2 L / \sqrt{L^{2}-1}$.

Proof. Let

$$
A=\left[\left(C_{1} \cap C_{2}\right)+(L r+\varepsilon) B_{X}\right] \cap\left[\left\{\left(C_{1}+r B_{X}\right) \cap C\right\}+\varepsilon B_{X}\right] \cap\left[\left\{\left(C_{2}+r B_{X}\right) \cap C\right\}+\varepsilon B_{X}\right]
$$

and let $a \in A$. Prove that

$$
a \in\left[\left\{\left(C_{1} \cap C_{2}\right)+L r B_{X}\right\} \cap C\right]+\theta(L) \varepsilon B_{X} .
$$

Let us show that if

$$
C_{1} \cap C_{2} \cap\left(C+r B_{X}\right) \cap B_{X}(a, L r+\varepsilon) \neq \emptyset
$$

then (2.23) holds. Indeed, (2.24) provides the existence of a point $x \in X$ such that

$$
x \in C_{1} \cap C_{2} \cap\left(C+r B_{X}\right) \cap B_{X}(a, L r+\varepsilon) .
$$

In particular, $x \in C+r B_{X}$ so that $B_{X}(x, r) \cap C \neq \emptyset$ proving that condition 2.6 of Theorem 2.3 holds. This theorem tells us that

$$
\left[B_{X}(x, L r) \cap C\right]+\theta(L) \varepsilon B_{X} \supset\left(C+\varepsilon B_{X}\right) \cap B_{X}(x, L r+\varepsilon) .
$$

Since $a \in A$, we have $a \in C+\varepsilon B_{X}$. From (2.25) we learn that and $a \in B_{X}(x, L r+\varepsilon)$. Thus, the point $a$ belongs to the set $\left(C+\varepsilon B_{X}\right) \cap B_{X}(x, L r+\varepsilon)$. Therefore, by (2.26),

$$
a \in\left[B_{X}(x, L r) \cap C\right]+\theta(L) \varepsilon B_{X}=\left[\left(x+L r B_{X}\right) \cap C\right]+\theta(L) \varepsilon B_{X} .
$$

But $x \in C_{1} \cap C_{2}$, see (2.25), and the required inclusion (2.23) follows. 
Thus, it remains to prove 2.24). Helly's Theorem 2.5 tells us that this property holds provided any three sets in the left hand side of (2.24) have a common point. Note that, thanks to (2.22), this is true for $C_{1}, C_{2}$ and $C+r B_{X}$. Since the point $a \in A$, we have $a \in\left[C_{1} \cap C_{2}\right]+(L r+\varepsilon) B_{X}$, so that $C_{1} \cap C_{2} \cap B_{X}(a, L r+\varepsilon) \neq \emptyset$.

Let us prove that

$$
C_{1} \cap\left(C+r B_{X}\right) \cap B_{X}(a, L r+\varepsilon) \neq \emptyset .
$$

The point $a \in A$ so that $a \in\left(C_{1}+r B_{X}\right) \cap C+\varepsilon B_{X}$. Let $b$ be a point nearest to $a$ on $\left(C_{1}+r B_{X}\right) \cap C$, and let $b_{1} \in C_{1}$ be a point nearest to $b$ on $C_{1}$. Prove that

$$
b_{1} \in C_{1} \cap\left(C+r B_{X}\right) \cap B_{X}(a, L r+\varepsilon) .
$$

Indeed, we have $\|a-b\| \leq \varepsilon$ and $\left\|b_{1}-b\right\| \leq r$. Thus,

$$
b_{1} \in C_{1} \quad \text { (by definition) and } \quad b_{1} \in C+r B_{X} \quad \text { (because } b \in C \text { ). }
$$

Furthermore,

$$
\left\|a-b_{1}\right\| \leq\|a-b\|+\left\|b-b_{1}\right\| \leq \varepsilon+r \leq \varepsilon+L r
$$

proving that $b_{1} \in B_{X}(a, L r+\varepsilon)$. Combining this property with 2.29) we obtain 2.28) and (2.27). In a similar way we show that $C_{2} \cap\left(C+r B_{X}\right) \cap B_{X}(a, L r+\varepsilon) \neq \emptyset$.

The proof of the proposition is complete.

\section{The main theorem for two dimensional Banach spaces.}

In this section we prove Theorem 1.9

First, let us recall the notion of the Lipschitz extension constant $e(\mathfrak{M}, X)$ which we use in the formulation of this theorem.

Definition 3.1. Let $\mathfrak{M}=(\mathcal{M}, \rho)$ be a pseudometric space, and let $X$ be a Banach space. We define the Lipschitz extension constant $e(\mathfrak{M}, X)$ of $X$ with respect to $\mathfrak{M}$ as the infimum of the constants $\lambda>0$ such that for every subset $\mathcal{M}^{\prime} \subset \mathcal{M}$, and every Lipschitz mapping $f: \mathcal{M}^{\prime} \rightarrow X$, there exists a Lipschitz extension $\tilde{f}: \mathcal{M} \rightarrow X$ of $f$ to all of $\mathcal{M}$ such that $\|\tilde{f}\|_{\operatorname{Lip}(\mathcal{M}, X)} \leq \lambda\|f\|_{\operatorname{Lip}\left(\mathcal{M}^{\prime}, X\right)}$.

Remark 3.2. Recall several results about Lipschitz extension constants which we use in this paper. In particular, thanks to the McShane-Whitney extension theorem, $e(\mathfrak{M}, \mathbf{R})=$ 1 for every pseudometric space $\mathfrak{M}=(\mathcal{M}, \rho)$. Hence, $e\left(\mathfrak{M}, \ell_{\infty}^{2}\right)=1$ as well.

It follows from [22] and [8] that $e(\mathfrak{M}, X) \leq 4 / 3$ provided $X$ is an arbitrary two dimensional Banach space. See also [3]. Furthermore, $e(\mathfrak{M}, X) \leq 4 / \pi$ whenever $X$ is an arbitrary two dimensional Euclidean space. See [22] and [17].

We also note that, thanks to Kirszbraun's extension theorem [18], $e(\mathfrak{M}, X)=1$ provided $X$ is a Euclidean space, $\mathcal{M}$ is a subset of a Euclidean space $E$, and $\rho$ is the Euclidean metric in $E$. 
Proof of Theorem 1.9. Let $\mathfrak{M}=(\mathcal{M}, \rho)$ be a pseudometric space, and let $X$ be a two dimensional Banach space. Let $F: \mathcal{M} \rightarrow \mathcal{K}(X)$ be a set-valued mapping satisfying the hypothesis of Theorem 1.9 . Recall that, by this hypothesis, for every subset $S \subset \mathcal{M}$ with $\# S \leq 4$, the restriction $\left.F\right|_{S}$ of $F$ to $S$ has a $\rho$-Lipschitz selection $f_{S}: S \rightarrow X$ with $\left\|f_{S}\right\|_{\operatorname{Lip}((S, \rho), X)} \leq 1$.

Let $\lambda_{1}$ and $\lambda_{2}$ be positive constants satisfying inequalities $(1.5)$, i.e., $\lambda_{1} \geq e(\mathfrak{M}, X)$ and $\lambda_{2} \geq 3 \lambda_{1}$. We set $L=\lambda_{2} / \lambda_{1}$. Thus, the following inequalities

$$
\lambda_{1} \geq e(\mathfrak{M}, X), \quad L \geq 3,
$$

hold. Then we introduce a new pseudometric on $\mathcal{M}$ defined by

$$
\mathrm{d}(x, y)=\lambda_{1} \rho(x, y), \quad x, y \in \mathcal{M} .
$$

This definition, Definition 3.1 , the above hypothesis of Theorem 1.9 and the inequality $\lambda_{1} \geq e(\mathfrak{M}, X)$ imply the following claim.

Claim 3.3. Let $\widetilde{S} \subset \mathcal{M}$ be a finite set, and let $S \subset \widetilde{S}$ be a set with $\# S \leq 4$. Then there exists a d-Lipschitz, mapping $\tilde{f}_{S}: \widetilde{S} \rightarrow X$ with $\left\|\tilde{f}_{S}\right\|_{\operatorname{Lip}(\widetilde{S}, \mathrm{~d}), X)} \leq 1$ such that $\tilde{f}_{S}(x) \in F(x)$ for every $x \in S$.

We introduce set-valued mappings

$$
F^{[1]}(x)=\bigcap_{z \in \mathcal{M}}\left[F(z)+\mathrm{d}(x, z) B_{X}\right], \quad x \in \mathcal{M},
$$

and

$$
F^{[2]}(x)=\bigcap_{z \in \mathcal{M}}\left[F^{[1]}(z)+L \mathrm{~d}(x, z) B_{X}\right], \quad x \in \mathcal{M} .
$$

Thus, $F^{[1]}$ and $F^{[2]}$ are the first and the second order $(\{1, L\}$, d)-balanced refinements of $F$ respectively. See Definition 1.6 .

Our aim is to show that, if $L$ and $\lambda_{1}$ satisfy inequality $\left[3.1\right.$, then (i) $F^{[2]}(x) \neq \emptyset$ on $\mathcal{M}$, and (ii) the mapping $F^{[2]}$ is d-Lipschitz with respect to the Hausdorff distance. We prove the statements (i) and (ii) in Proposition 3.8 and Proposition 3.10 respectively.

We begin with the property (i). Its proof relies on a series of auxiliary lemmas.

Lemma 3.4. Let $X$ be a two dimensional Banach space, and let $\mathcal{K} \subset \mathcal{K}(X)$ be a collection of convex compact subsets of $X$ with non-empty intersection.

Given $\tau>0$, let $B=\tau B_{X}$. Then

$$
\left(\bigcap_{K \in \mathcal{K}} K\right)+B=\bigcap_{K, K^{\prime} \in \mathcal{K}}\left\{\left(K \cap K^{\prime}\right)+B\right\} .
$$

Proof. Obviously, the right hand side of (3.5) contains its left hand side. Let us prove the converse statement. Fix a point

$$
x \in \bigcap_{K, K^{\prime} \in \mathcal{K}}\left\{\left(K \cap K^{\prime}\right)+B\right\}
$$


and prove that $x \in \cap\{K: K \in \mathcal{K}\}+B$.

Clearly, it is true if $B_{X}(x, \tau) \cap(\cap\{K: K \in \mathcal{K}\}) \neq \emptyset$.

Let $S=\mathcal{K} \cup\left\{B_{X}(x, \tau)\right\}$. Helly's intersection Theorem 2.5 tells us that this property holds provided $\cap\left\{K: K \in S^{\prime}\right\} \neq \emptyset$ for every subfamily $S^{\prime} \subset S$ consisting of at most three elements. Clearly, this is true if $B_{X}(x, \tau) \notin S^{\prime}$ because there exists a point common to all of the sets from $\mathcal{K}$.

Suppose that $B_{X}(x, \tau) \in S^{\prime}$. Then $S^{\prime}=\left\{B_{X}(x, \tau), K, K^{\prime}\right\}$ for some $K, K^{\prime} \in \mathcal{K}$. Thanks to (3.6), $x \in\left(K \cap K^{\prime}\right)+B$ so that $B_{X}(x, \tau) \cap K \cap K^{\prime} \neq \emptyset$.

Thus, $B_{X}(x, \tau) \cap(\cap\{K: K \in \mathcal{K}\}) \neq \emptyset$, and the proof of the lemma is complete.

Lemma 3.5. For each $x \in \mathcal{M}$ the set $F^{[1]}(x) \in \mathcal{K}(X)$, i.e., $F^{[1]}(x)$ is a non-empty convex compact subset of $X$. Furthermore, for every $x, z \in \mathcal{M}$ we have

$F^{[1]}(z)+L \mathrm{~d}(x, z) B_{X}=\bigcap_{y^{\prime}, y^{\prime \prime} \in \mathcal{M}}\left\{\left[\left(F\left(y^{\prime}\right)+\mathrm{d}\left(z, y^{\prime}\right) B_{X}\right) \cap\left(F\left(y^{\prime \prime}\right)+\mathrm{d}\left(z, y^{\prime \prime}\right) B_{X}\right)\right]+L \mathrm{~d}(x, z) B_{X}\right\}$.

Proof. Prove that $F^{[1]}(x) \neq \emptyset$ for every $x \in \mathcal{M}$. Indeed, formula $(3.3)$ and Helly's Theorem 2.5 tell us that $F^{[1]}(x) \neq \emptyset$ provided

$$
\left[F\left(z_{1}\right)+\mathrm{d}\left(x, z_{1}\right) B_{X}\right] \cap\left[F\left(z_{2}\right)+\mathrm{d}\left(x, z_{2}\right) B_{X}\right] \cap\left[F\left(z_{3}\right)+\mathrm{d}\left(x, z_{3}\right) B_{X}\right] \neq \emptyset
$$

for every $z_{1}, z_{2}, z_{3} \in \mathcal{M}$.

To prove this property, we apply the hypothesis of Theorem 1.9 to the set $S=$ $\left\{x, z_{1}, z_{2}, z_{3}\right\}$. Thanks to this hypothesis, the restriction $\left.F\right|_{S}$ has a $\rho$-Lipschitz selection $f_{S}: S \rightarrow X$ with $\rho$-Lipschitz seminorm at most 1 . Hence,

$$
\left\|f_{S}(x)-f_{S}\left(z_{i}\right)\right\| \leq \rho\left(x, z_{i}\right) \leq \lambda_{1} \rho\left(x, z_{i}\right)=\mathrm{d}\left(x, z_{i}\right) \text { for every } i=1,2,3,
$$

proving that $f_{S}(x)$ belongs to the left hand side of (3.7). Thus, (3.7) holds for arbitrary $z_{i} \in \mathcal{M}, i=1,2,3$, so that $F^{[1]}(x) \neq \emptyset$.

Clearly, $F^{[1]}(x)$ is a convex bounded subset of $X$. See $(3.3)$.

Finally, the second statement of the lemma immediately follows from this property, Lemma 3.4 and formula (3.3). The proof of the lemma is complete.

Lemma 3.6. For every $x \in \mathcal{M}$ the set $F^{[2]}(x)$ admits the following representation:

$$
F^{[2]}(x)=\bigcap_{u, u^{\prime}, u^{\prime \prime} \in \mathcal{M}}\left\{\left[\left(F\left(u^{\prime}\right)+\mathrm{d}\left(u^{\prime}, u\right) B_{X}\right) \cap\left(F\left(u^{\prime \prime}\right)+\mathrm{d}\left(u^{\prime \prime}, u\right) B_{X}\right)\right]+L \mathrm{~d}(u, x) B_{X}\right\} .
$$

Proof. The lemma is immediate from (3.4) and Lemma 3.5 .

Given $x, u, u^{\prime}, u^{\prime \prime} \in \mathcal{M}$ we set

$$
T_{x}\left(u, u^{\prime}, u^{\prime \prime}\right)=\left[\left(F\left(u^{\prime}\right)+\mathrm{d}\left(u^{\prime}, u\right) B_{X}\right) \bigcap\left(F\left(u^{\prime \prime}\right)+\mathrm{d}\left(u^{\prime \prime}, u\right) B_{X}\right)\right]+L \mathrm{~d}(u, x) B_{X} .
$$

Remark 3.7. Let us recall the standard definitions of a metric tree. Let $T=(V, E)$ be a finite (graph theoretic) tree, where $V$ denotes the set of nodes of $T$, and $E$ denotes the set of edges. Suppose we assign a positive number $\Delta(e)$ to each edge $e \in E$. Then for $x, y \in V$ we can define their distance $\mathrm{d}_{T}(x, y)$ to be the sum of $\Delta(e)$ over all the edges $e$ in the "minimal path" joining $x$ to $y$ as in Fig. 12 . 


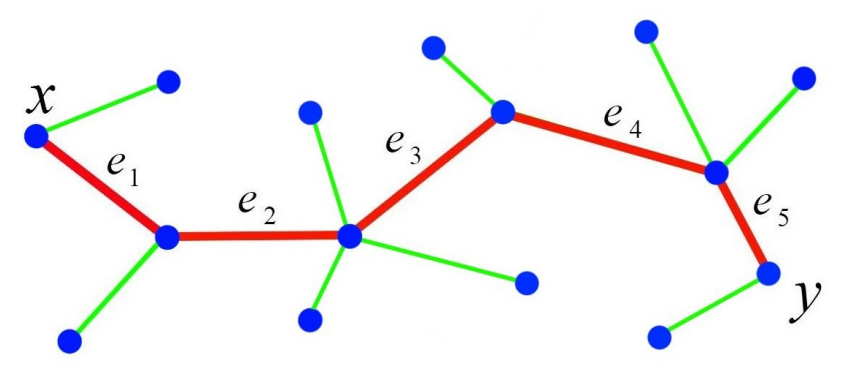

Fig. 12: A minimal path joining nodes $x$ and $y$ in a tree. In this case, $d(x, y)=\Delta\left(e_{1}\right)+$ $\Delta\left(e_{2}\right)+\ldots+\Delta\left(e_{5}\right)$.

We call $\mathrm{d}_{T}$ a tree metric; $\left(V, \mathrm{~d}_{T}\right)$ is a metric tree. We say that a mapping $g: V \rightarrow X$ agrees with the metric tree $\mathfrak{M}_{T}=\left(V, \mathrm{~d}_{T}\right)$ if the Lipschitz seminorm $\|g\|_{\operatorname{Lip}\left(\left(V, \mathrm{~d}_{T}\right), X\right)} \leq 1$.

In these settings, given $x, u, u^{\prime}, u^{\prime \prime} \in \mathcal{M}$, the set $T_{x}\left(u, u^{\prime}, u^{\prime \prime}\right)$ defined by (3.8) admits the following equivalent definition: Let $T$ be the metric tree with the family of nodes $V=\left\{x, u, u^{\prime}, u^{\prime \prime}\right\}$ and the set of edges $E=\left\{e_{1}=[u, x], e_{2}=\left[u^{\prime}, u\right], e_{3}=\left[u^{\prime \prime}, u\right]\right\}$ and the weights $w\left(e_{1}\right)=L \mathrm{~d}(u, x), w\left(e_{2}\right)=\mathrm{d}\left(u^{\prime}, u\right)$ and $w\left(e_{3}\right)=\mathrm{d}\left(u^{\prime \prime}, u\right)$, as it shown in Fig. 13 below.

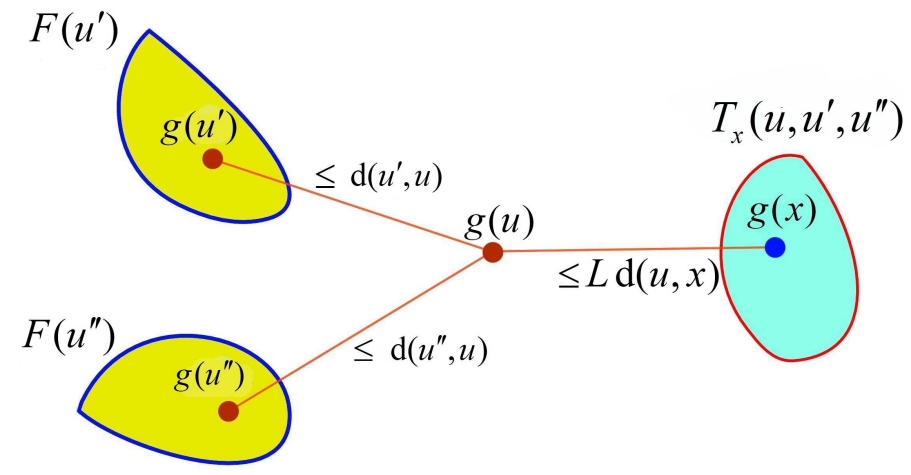

Fig. 13: $T_{x}\left(u, u^{\prime}, u^{\prime \prime}\right)$ is the orbit of $x$ with respect to this diagram.

Then $T_{x}\left(u, u^{\prime}, u^{\prime \prime}\right)=\{g(x)\}$ where $g$ runs over all Lipschitz mappings which agree with the metric tree $\mathfrak{M}_{T}=\left(V, \mathrm{~d}_{T}\right)$ such that $g\left(u^{\prime}\right) \in F\left(u^{\prime}\right)$ and $g\left(u^{\prime \prime}\right) \in F\left(u^{\prime \prime}\right)$. We say that the set $T_{x}\left(u, u^{\prime}, u^{\prime \prime}\right)$ is the orbit of $x$ with respect to the diagram shown in Fig. 13.

In these settings, Lemma 3.6 reformulates as follows:

$$
F^{[2]}(x)=\bigcap_{u, u^{\prime}, u^{\prime \prime} \in \mathcal{M}} T_{x}\left(u, u^{\prime}, u^{\prime \prime}\right) .
$$

Proposition 3.8. For every $x \in \mathcal{M}$ the set $F^{[2]}(x)$ is non-empty.

Proof. Formula (3.9) and Helly's Theorem 2.5 tell us that $F^{[2]}(x) \neq \emptyset$ provided for every choice of elements $u_{i}, u_{i}^{\prime}, u_{i}^{\prime \prime} \in \mathcal{M}, i=1,2,3$, we have

$$
T_{x}\left(u_{1}, u_{1}^{\prime}, u_{1}^{\prime \prime}\right) \cap T_{x}\left(u_{2}, u_{2}^{\prime}, u_{2}^{\prime \prime}\right) \cap T_{x}\left(u_{3}, u_{3}^{\prime}, u_{3}^{\prime \prime}\right) \neq \emptyset .
$$


We note that 3.10 holds provided there exists a mapping $g$ which agrees with the diagram shown in Fig. 14 below.

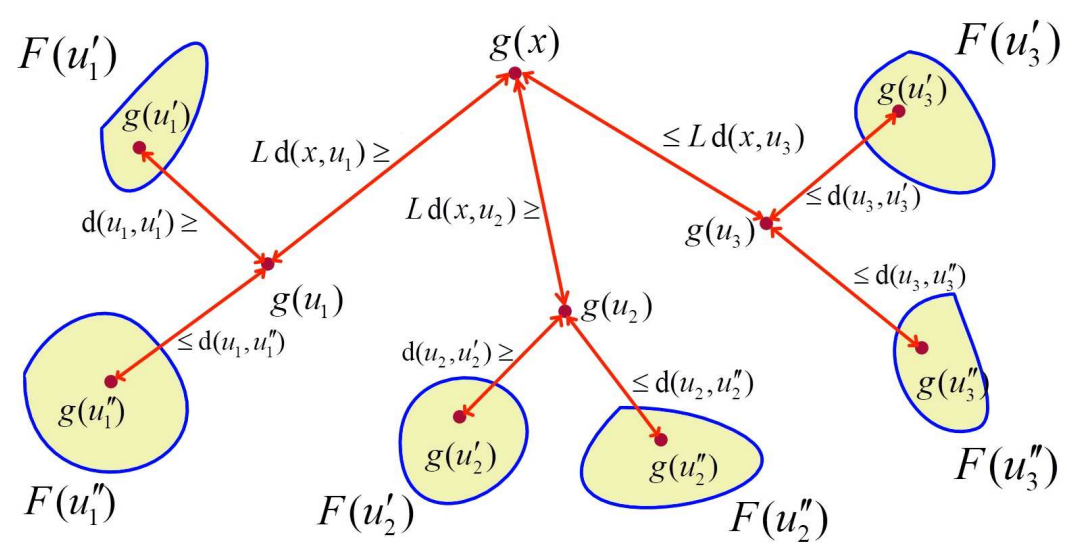

Fig. 14: The mapping $g$ agrees with this diagram.

We set $r_{i}=\mathrm{d}\left(x, u_{i}\right), i=1,2,3$. Without loss of generality, we may assume that $r_{1} \leq r_{2} \leq r_{3}$. For each $i \in\{1,2,3\}$ we also set

$$
G\left(u_{i}^{\prime}\right)=F\left(u_{i}^{\prime}\right)+\mathrm{d}\left(u_{i}^{\prime}, u_{i}\right) B_{X} \quad \text { and } \quad G\left(u_{i}^{\prime \prime}\right)=F\left(u_{i}^{\prime \prime}\right)+\mathrm{d}\left(u_{i}^{\prime \prime}, u_{i}\right) B_{X} .
$$

Note that the existence of a mapping $g$ on the set $\left\{x, u_{i}, u_{i}^{\prime}, u_{i}^{\prime \prime}: i=1,2,3\right\}$ which agrees with the diagram in Fig. 14 is equivalent to the existence of a mapping $g$ on $\left\{x, u_{1}, u_{2}, u_{3}\right\}$ which agrees with the diagram shown in the following Fig. 15.

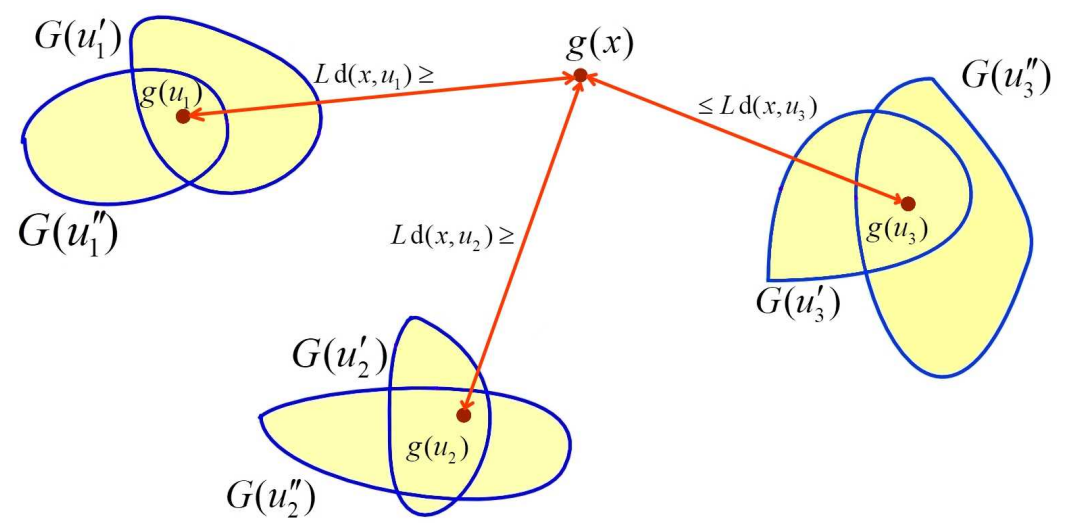

Fig. 15: The mapping $g:\left\{x, u_{1}, u_{2}, u_{3}\right\} \rightarrow X$ agrees with this diagram.

Let us prove that there exist points $y_{i} \in X, i=1,2,3$, such that

$$
y_{i} \in G\left(u_{i}^{\prime}\right) \cap G\left(u_{i}^{\prime \prime}\right) \quad \text { for every } \quad i=1,2,3 \text {, }
$$

and

$$
\left\|y_{1}-y_{2}\right\| \leq r_{1}+r_{2} \quad \text { and } \quad\left\|y_{1}-y_{3}\right\| \leq r_{1}+2 r_{2}+r_{3} . \quad \text { See Fig. } 16
$$




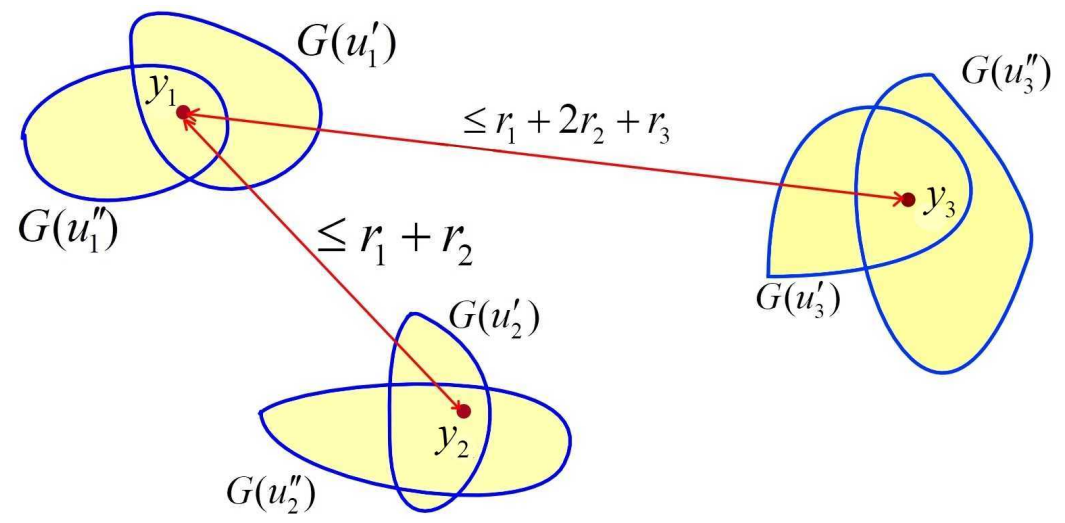

Fig. 16: The existence of points $\left\{y_{i}: i=1,2,3\right\}$ implies property (3.10).

Let us see that the existence of the points $y_{i}$ with these properties implies (3.10). Indeed, since $\left\|y_{1}-y_{3}\right\| \leq r_{1}+2 r_{2}+r_{3}$, there exists $z \in\left[y_{1}, y_{3}\right]$ such that $\left\|z-y_{1}\right\| \leq r_{1}$ and $\left\|z-y_{3}\right\| \leq 2 r_{2}+r_{3}$. (For instance, one can set $z=y_{1}+\tau\left(y_{3}-y_{1}\right)$ with $\tau=r_{1} /\left(r_{1}+2 r_{2}+r_{3}\right)$.)

Hence,

$$
\left\|y_{2}-z\right\| \leq\left\|y_{2}-y_{1}\right\|+\left\|y_{1}-z\right\| \leq r_{1}+r_{2}+r_{1}=2 r_{1}+r_{2} .
$$

Recall that $r_{i}=\mathrm{d}\left(x, u_{i}\right)$ and $r_{1} \leq r_{2} \leq r_{3}$. From this and above inequalities, we have

$$
\left\|z-y_{i}\right\| \leq 3 r_{i}=3 \mathrm{~d}\left(x, u_{i}\right), \quad i=1,2,3 .
$$

Let us prove that $z \in T_{x}\left(u_{i}, u_{i}^{\prime}, u_{i}^{\prime \prime}\right)$ for each $i \in\{1,2,3\}$. In fact, we know that $L \geq 3$, see (3.1). Furthermore, by (3.12), $y_{i} \in G\left(u_{i}^{\prime}\right) \cap G\left(u_{i}^{\prime \prime}\right)$, so that, thanks to (3.11), 3.14) and definition (3.8),

$$
z \in\left[G\left(u_{i}^{\prime}\right) \cap G\left(u_{i}^{\prime \prime}\right)\right]+3 \mathrm{~d}\left(x, u_{i}\right) B_{X} \subset\left[G\left(u_{i}^{\prime}\right) \cap G\left(u_{i}^{\prime \prime}\right)\right]+L \mathrm{~d}\left(x, u_{i}\right) B_{X}=T_{x}\left(u_{i}, u_{i}^{\prime}, u_{i}^{\prime \prime}\right)
$$

proving 3.10.

Thus, our aim is to prove the existence of points $y_{i}$ satisfying (3.12) and (3.13). We will do this in three steps.

STEP 1 . We introduce sets $W_{i} \subset X, i=1, \ldots, 4$, defined by

$$
W_{1}=G\left(u_{1}^{\prime}\right), \quad W_{2}=G\left(u_{1}^{\prime \prime}\right), \quad W_{3}=\left[G\left(u_{2}^{\prime}\right) \bigcap G\left(u_{2}^{\prime \prime}\right)\right]+\left(r_{1}+r_{2}\right) B_{X},
$$

and

$$
W_{4}=\left[G\left(u_{3}^{\prime}\right) \bigcap G\left(u_{3}^{\prime \prime}\right)\right]+\left(r_{1}+2 r_{2}+r_{3}\right) B_{X} .
$$

Obviously, there exist the points $y_{i}$ satisfying 3.12 and 3.13 whenever

$$
W_{1} \cap W_{2} \cap W_{3} \cap W_{4} \neq \emptyset .
$$

See Fig. 17. 


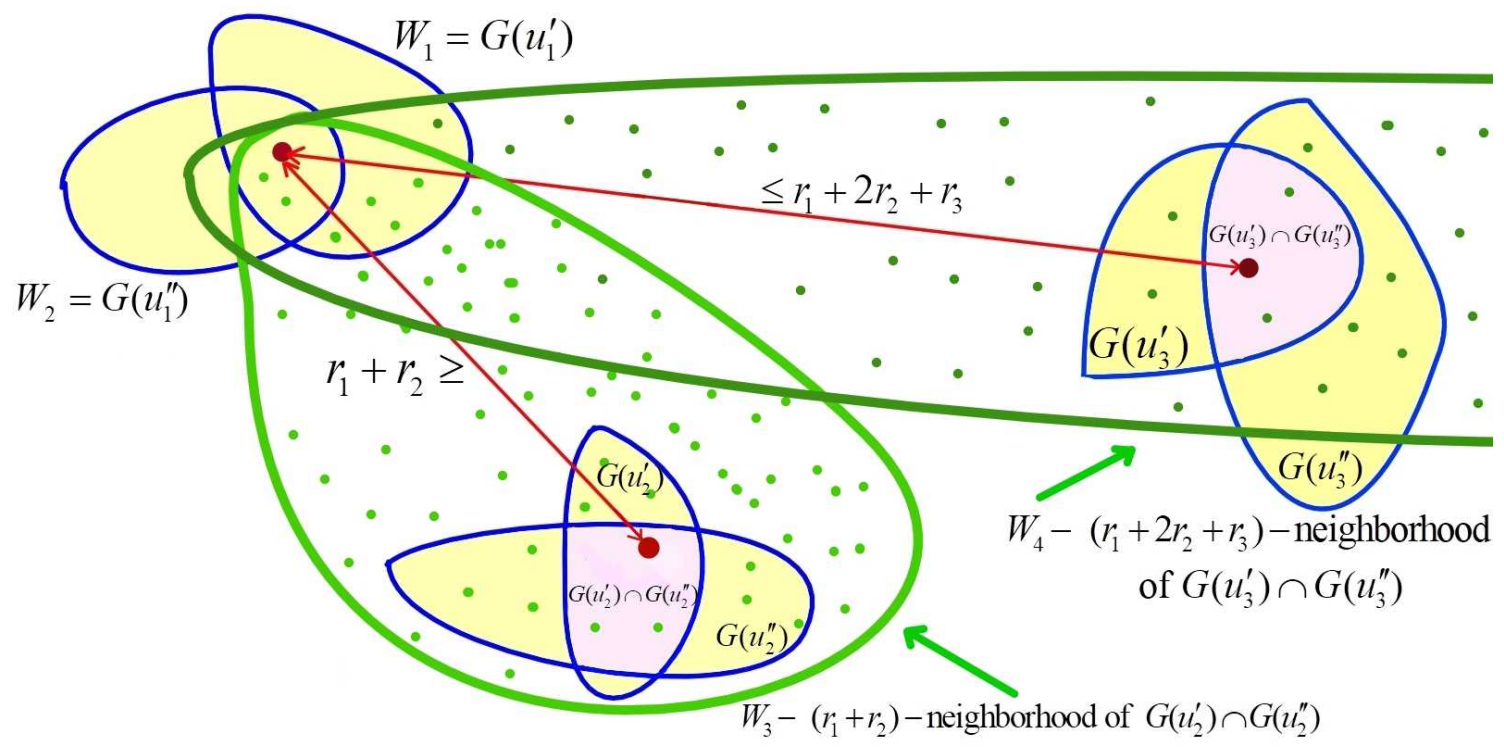

Fig. 17: The sets $W_{1}, W_{2}, W_{3}$ and $W_{4}$.

By Helly's Theorem 2.5, this property holds provided any three members of the family of sets $\left\{W_{1}, W_{2}, W_{3}, W_{4}\right\}$ have a common point.

STEP 2. Prove that $W_{1} \cap W_{3} \cap W_{4} \neq \emptyset$. To see this, we set

$$
V_{1}=G\left(u_{1}^{\prime}\right)+\left(r_{1}+r_{2}\right) B_{X}, \quad V_{2}=G\left(u_{2}^{\prime}\right), \quad V_{3}=G\left(u_{2}^{\prime \prime}\right),
$$

and

$$
V_{4}=\left[G\left(u_{3}^{\prime}\right) \cap G\left(u_{3}^{\prime \prime}\right)\right]+\left(r_{2}+r_{3}\right) B_{X} .
$$

Let us show that if

$$
V_{1} \cap V_{2} \cap V_{3} \cap V_{4} \neq \emptyset,
$$

then $W_{1} \cap W_{3} \cap W_{4}$ is non-empty as well. Indeed, this property, definitions (3.17) and (3.18) imply the existence of points $z_{1} \in G\left(u_{1}^{\prime}\right), z_{2} \in G\left(u_{2}^{\prime}\right) \cap G\left(u_{2}^{\prime \prime}\right), z_{3} \in G\left(u_{3}^{\prime}\right) \cap G\left(u_{3}^{\prime \prime}\right)$ such that $\left\|z_{1}-z_{2}\right\| \leq r_{1}+r_{2}$ and $\left\|z_{2}-z_{3}\right\| \leq r_{2}+r_{3}$. Hence,

$$
\left\|z_{1}-z_{3}\right\| \leq\left\|z_{1}-z_{2}\right\|+\left\|z_{2}-z_{3}\right\| \leq\left(r_{1}+r_{2}\right)+\left(r_{2}+r_{3}\right)=r_{1}+2 r_{2}+r_{3} .
$$

Thus, thanks to (3.15) and (3.16), the point $z_{1}$ belongs to $W_{1} \cap W_{3} \cap W_{4}$ proving that this set is non-empty. smsk

Let us prove (3.19). Helly's Theorem 2.5 tells us that 3.19 holds whenever every three members of the family $\mathcal{V}=\left\{V_{1}, V_{2}, V_{3}, V_{4}\right\}$ have a common point.

Let us prove this property. First, let us show that

$$
V_{1} \cap V_{2} \cap V_{4} \neq \emptyset .
$$

Let $S=\left\{u_{1}^{\prime}, u_{2}^{\prime}, u_{3}^{\prime}, u_{3}^{\prime \prime}\right\}$ and let $\widetilde{S}=\left\{u_{1}^{\prime}, u_{2}, u_{2}^{\prime}, u_{3}, u_{3}^{\prime}, u_{3}^{\prime \prime}\right\}$. Note that 3.20 holds if and only if there exists a mapping $\tilde{g}$ on $\widetilde{S}$ which agrees with the diagram in Fig. 18 below. 


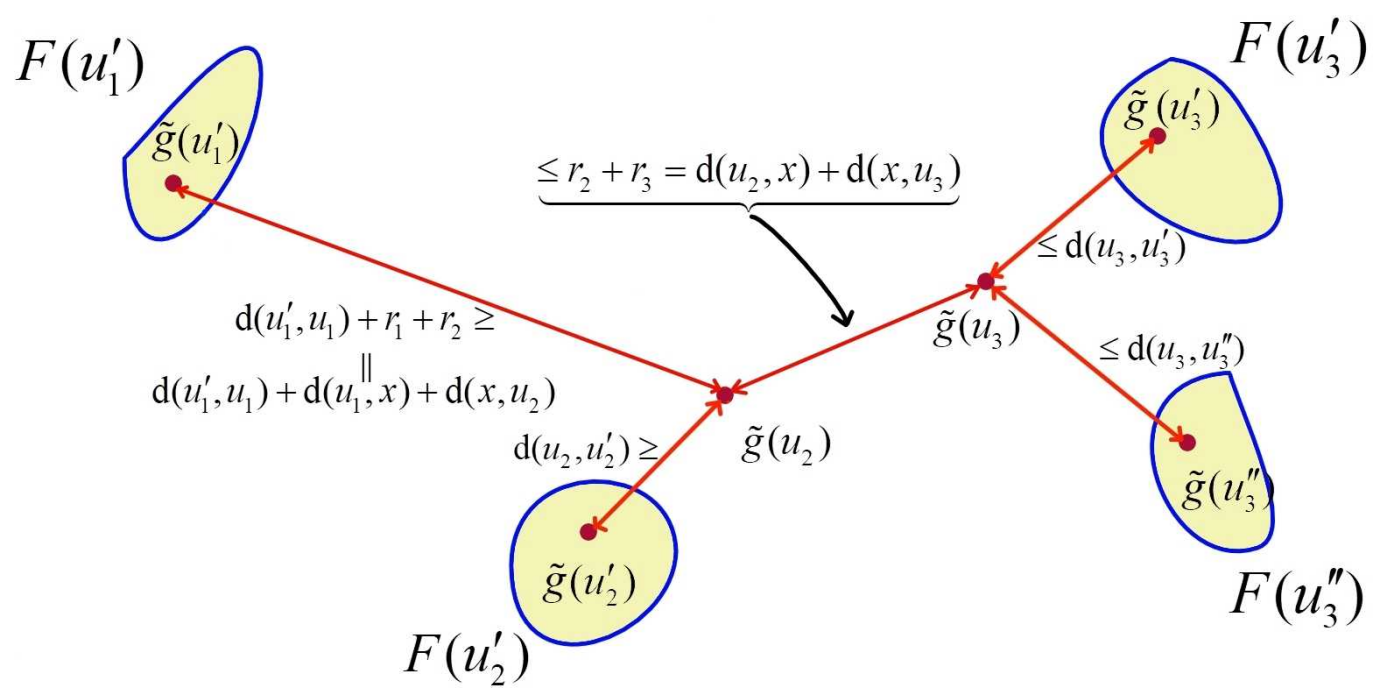

Fig. 18: The mapping $\tilde{g}: \widetilde{S} \rightarrow X$ agrees with this diagram.

Note that, thanks to Claim 3.3 , there exists a d-Lipschitz mapping $\tilde{f}_{S}: \widetilde{S} \rightarrow X$ with $\left\|\tilde{f}_{S}\right\|_{\operatorname{Lip}((\widetilde{S}, \mathrm{~d}), X)} \leq 1$ such that

$$
\tilde{f}_{S}\left(u_{1}^{\prime}\right) \in F\left(u_{1}^{\prime}\right), \quad \tilde{f}_{S}\left(u_{2}^{\prime}\right) \in F\left(u_{2}^{\prime}\right), \quad \tilde{f}_{S}\left(u_{3}^{\prime}\right) \in F\left(u_{3}^{\prime}\right), \quad \text { and } \quad \tilde{f}_{S}\left(u_{3}^{\prime \prime}\right) \in F\left(u_{3}^{\prime \prime}\right) .
$$

Prove that

$$
\tilde{f}_{S}\left(u_{2}\right) \in V_{1} \cap V_{2} \cap V_{4} .
$$

Indeed, $\tilde{f}_{S}\left(u_{2}^{\prime}\right) \in F\left(u_{2}^{\prime}\right)$ and $\left\|\tilde{f}_{S}\left(u_{2}^{\prime}\right)-\tilde{f}_{S}\left(u_{2}\right)\right\| \leq \mathrm{d}\left(u_{2}^{\prime}, u_{2}\right)$, so that

$$
\tilde{f}_{S}\left(u_{2}\right) \in F\left(u_{2}^{\prime}\right)+\mathrm{d}\left(u_{2}^{\prime}, u_{2}\right) B_{X}=G\left(u_{2}^{\prime}\right)=V_{2} .
$$

In the same way we prove that $\tilde{f}_{S}\left(u_{1}\right) \in G\left(u_{1}^{\prime}\right)$.

Note that $\left\|\tilde{f}_{S}\left(u_{1}\right)-\tilde{f}_{S}\left(u_{2}\right)\right\| \leq \mathrm{d}\left(u_{1}, u_{2}\right)$ so that $\tilde{f}_{S}\left(u_{2}\right) \in G\left(u_{1}^{\prime}\right)+\mathrm{d}\left(u_{1}, u_{2}\right) B_{X}$. By the triangle inequality,

$$
\mathrm{d}\left(u_{1}, u_{2}\right) \leq \mathrm{d}\left(u_{1}, x\right)+\mathrm{d}\left(x, u_{2}\right)=r_{1}+r_{2}
$$

proving that

$$
\tilde{f}_{S}\left(u_{2}\right) \in G\left(u_{1}^{\prime}\right)+\left(r_{1}+r_{2}\right) B_{X}=V_{1} .
$$

It remains to show that $\tilde{f}_{S}\left(u_{2}\right)$ belongs to $V_{4}$. We know that $\tilde{f}_{S}\left(u_{3}^{\prime}\right) \in F\left(u_{3}^{\prime}\right)$ and $\tilde{f}_{S}\left(u_{3}^{\prime \prime}\right) \in F\left(u_{3}^{\prime \prime}\right)$. Furthermore,

$$
\left\|\tilde{f}_{S}\left(u_{3}\right)-\tilde{f}_{S}\left(u_{3}^{\prime}\right)\right\| \leq \mathrm{d}\left(u_{3}, u_{3}^{\prime}\right), \quad\left\|\tilde{f}_{S}\left(u_{3}\right)-\tilde{f}_{S}\left(u_{3}^{\prime \prime}\right)\right\| \leq \mathrm{d}\left(u_{3}, u_{3}^{\prime \prime}\right) .
$$

Hence,

$$
\tilde{f}_{S}\left(u_{3}\right) \in\left[F\left(u_{3}^{\prime}\right)+\mathrm{d}\left(u_{3}^{\prime}, u_{3}\right) B_{X}\right] \cap\left[F\left(u_{3}^{\prime \prime}\right)+\mathrm{d}\left(u_{3}^{\prime \prime}, u_{3}\right) B_{X}\right]=G\left(u_{3}^{\prime}\right) \cap G\left(u_{3}^{\prime \prime}\right) .
$$

Furthermore, $\left\|\tilde{f}_{S}\left(u_{2}\right)-\tilde{f}_{S}\left(u_{3}\right)\right\| \leq \mathrm{d}\left(u_{2}, u_{3}\right)$. These properties of $\tilde{f}_{S}\left(u_{3}\right)$ and the triangle inequality

$$
\mathrm{d}\left(u_{2}, u_{3}\right) \leq \mathrm{d}\left(u_{2}, x\right)+\mathrm{d}\left(x, u_{3}\right)=r_{2}+r_{3}
$$


imply the following:

$$
\tilde{f}_{S}\left(u_{2}\right) \in\left[G\left(u_{3}^{\prime}\right) \bigcap G\left(u_{3}^{\prime \prime}\right)\right]+\mathrm{d}\left(u_{2}, u_{3}\right) B_{X} \subset\left[G\left(u_{3}^{\prime}\right) \bigcap G\left(u_{3}^{\prime \prime}\right)\right]+\left(r_{2}+r_{3}\right) B_{X}=V_{4} .
$$

Thus, $\tilde{f}_{S}\left(u_{2}\right) \in V_{1} \cap V_{2} \cap V_{4}$ proving (3.20).

In the same fashion we show that $V_{1} \cap V_{3} \cap V_{4} \neq \emptyset$.

Next, let us prove that

$$
V_{2} \cap V_{3} \cap V_{4}=G\left(u_{2}^{\prime}\right) \bigcap G\left(u_{2}^{\prime \prime}\right) \bigcap\left\{\left[G\left(u_{3}^{\prime}\right) \bigcap G\left(u_{3}^{\prime \prime}\right)\right]+\left(r_{2}+r_{3}\right) B_{X}\right\} \neq \emptyset .
$$

Following the scheme of the proof of $(3.20)$, we put

$$
S=\left\{u_{2}^{\prime}, u_{2}^{\prime \prime}, u_{3}^{\prime}, u_{3}^{\prime \prime}\right\} \quad \text { and } \quad \widetilde{S}=\left\{u_{2}, u_{2}^{\prime}, u_{2}^{\prime \prime}, u_{3}, u_{3}^{\prime}, u_{3}^{\prime \prime}\right\}
$$

We note that 3.22 holds provided there exists a mapping $\tilde{g}: \widetilde{S} \rightarrow X$ which agrees with the diagram on Fig. 19 below.

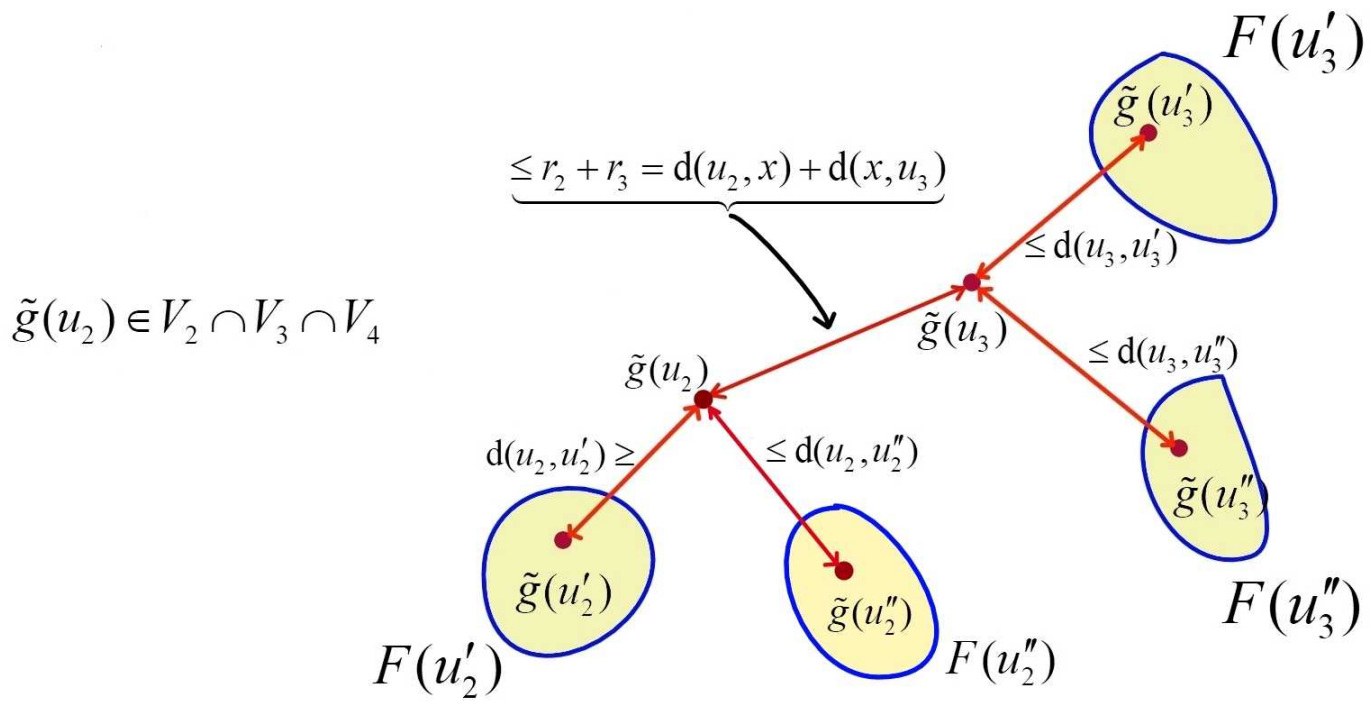

Fig. 19: The existence of $\tilde{g}: \widetilde{S} \rightarrow X$ which agrees with this diagram implies 3.22).

Claim 3.3 tells us that there exists a d-Lipschitz mapping $\tilde{f}_{S}: \widetilde{S} \rightarrow X$ with Lipschitz seminorm $\left\|\tilde{f}_{S}\right\|_{\operatorname{Lip}(\widetilde{S}, \mathrm{~d}), X)} \leq 1$ such that $\tilde{f}_{S}\left(u_{i}^{\prime}\right) \in F\left(u_{i}^{\prime}\right)$ and $\tilde{f}_{S}\left(u_{i}^{\prime \prime}\right) \in F\left(u_{i}^{\prime \prime}\right), i=2,3$.

Then, following the scheme of the proof of 3.21 , we show that $\tilde{f}_{S}\left(u_{2}\right)$ belongs to $V_{2} \cap V_{3} \cap V_{4}$ proving the required property (3.22).

Finally, following the same approach, we prove that

$$
V_{1} \cap V_{2} \cap V_{3}=\left[G\left(u_{1}^{\prime}\right)+\left(r_{1}+r_{2}\right) B_{X}\right] \cap G\left(u_{2}^{\prime}\right) \cap G\left(u_{2}^{\prime \prime}\right) \neq \emptyset .
$$

More specifically, we set $S=\left\{u_{1}^{\prime}, u_{2}, u_{2}^{\prime}, u_{2}^{\prime \prime}\right\}$. Then, thanks to the hypothesis of Theorem 1.9, there exists a d-Lipschitz mapping $\tilde{f}_{S}: S \rightarrow X$ with $\left\|\tilde{f}_{S}\right\|_{\operatorname{Lip}((S, \mathrm{~d}), X)} \leq 1$ such that

$$
\tilde{f}_{S}\left(u_{1}^{\prime}\right) \in F\left(u_{1}^{\prime}\right), \quad \tilde{f}_{S}\left(u_{2}^{\prime}\right) \in F\left(u_{2}^{\prime}\right) \text { and } \tilde{f}_{S}\left(u_{2}^{\prime \prime}\right) \in F\left(u_{2}^{\prime \prime}\right)
$$


Following the proof of 3.21 , we show that $\tilde{f}_{S}\left(u_{2}\right) \in V_{1} \cap V_{2} \cap V_{3}$ completing the proof of (3.23).

Thus, (3.19) is proven, so that $W_{1} \cap W_{3} \cap W_{4} \neq \emptyset$.

STEP 3. First, using a similar approach, we show that $W_{2} \cap W_{3} \cap W_{4} \neq \emptyset$.

Next, we prove that

$$
W_{1} \cap W_{2} \cap W_{4}=G\left(u_{1}^{\prime}\right) \cap G\left(u_{1}^{\prime \prime}\right) \bigcap\left\{\left[G\left(u_{3}^{\prime}\right) \cap G\left(u_{3}^{\prime \prime}\right)\right]+\left(r_{1}+2 r_{2}+r_{3}\right) B_{X}\right\} \neq \emptyset
$$

To see this, we set $S=\left\{u_{1}^{\prime}, u_{1}^{\prime \prime}, u_{3}^{\prime}, u_{3}^{\prime \prime}\right\}$ and $\widetilde{S}=\left\{u_{1}, u_{1}^{\prime}, u_{1}^{\prime \prime}, u_{3}, u_{3}^{\prime}, u_{3}^{\prime \prime}\right\}$. Note that 3.24 holds whenever the distance between the set $G\left(u_{1}^{\prime}\right) \cap G\left(u_{1}^{\prime \prime}\right)$ and the set $G\left(u_{3}^{\prime}\right) \cap G\left(u_{3}^{\prime \prime}\right)$ is bounded by $r_{1}+r_{2}+r_{3}$. See Fig. 20 .

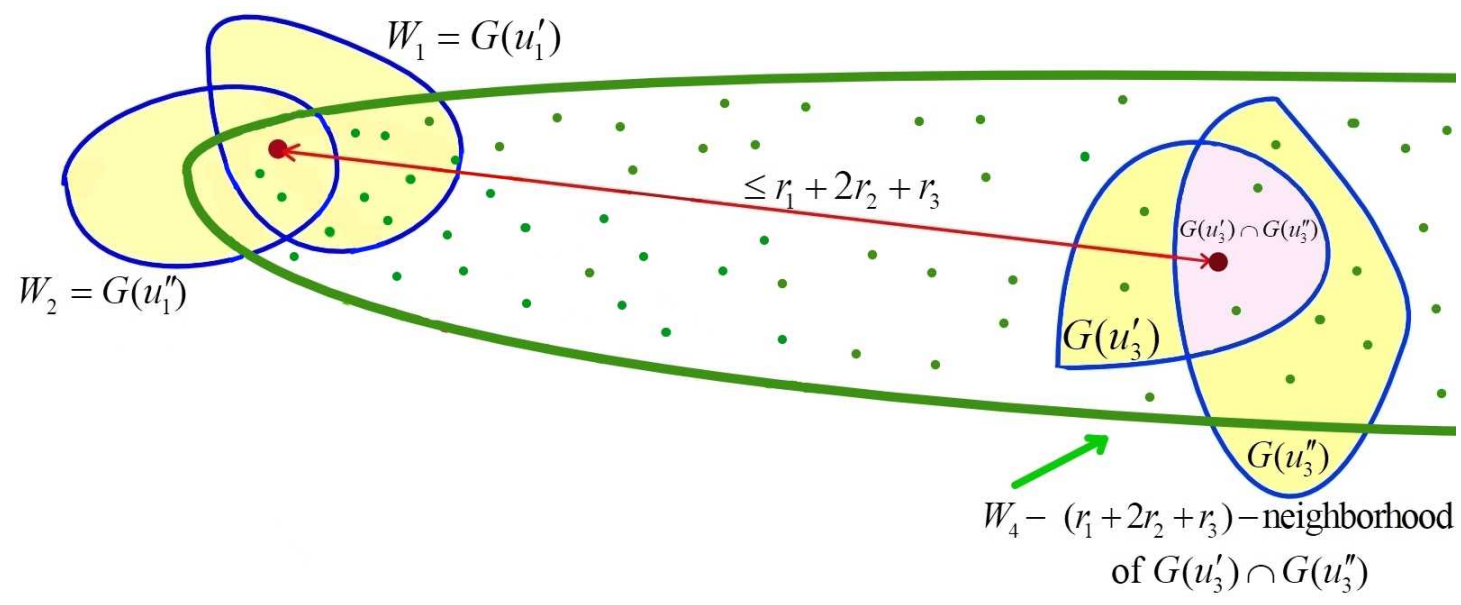

Fig. 20: The sets $W_{1}, W_{2}$ and $W_{4}$.

We note that Claim 3.3 tells us that there exists a d-Lipschitz mapping $\tilde{f}_{S}: \widetilde{S} \rightarrow X$ with $\left\|\tilde{f}_{S}\right\|_{\operatorname{Lip}(\widetilde{S}, \mathrm{~d}), X)} \leq 1$ such that $\tilde{f}_{S}\left(u_{i}^{\prime}\right) \in F\left(u_{i}^{\prime}\right)$ and $\tilde{f}_{S}\left(u_{i}^{\prime \prime}\right) \in F\left(u_{i}^{\prime \prime}\right), i=1,3$. The reader can easily see that the point $\tilde{f}_{S}\left(u_{1}\right)$ belongs to $W_{1} \cap W_{2} \cap W_{4}$ so that 3.24 holds.

In a similar way we prove that

$$
W_{1} \cap W_{2} \cap W_{3}=G\left(u_{1}^{\prime}\right) \cap G\left(u_{1}^{\prime \prime}\right) \bigcap\left\{\left[G\left(u_{2}^{\prime}\right) \cap G\left(u_{2}^{\prime \prime}\right)\right]+\left(r_{1}+r_{2}\right) B_{X}\right\} \neq \emptyset .
$$

More specifically, we set $S=\left\{u_{1}^{\prime}, u_{1}^{\prime \prime}, u_{2}^{\prime}, u_{2}^{\prime \prime}\right\}, \widetilde{S}=\left\{u_{1}, u_{1}^{\prime}, u_{1}^{\prime \prime}, u_{2}, u_{2}^{\prime}, u_{2}^{\prime \prime}\right\}$, and apply Claim 3.3 to $S$ and $\widetilde{S}$. Thanks to this claim, there exists a d-Lipschitz mapping $\tilde{f}_{S}$ on $\widetilde{S}$ with $\left\|\tilde{f}_{S}\right\|_{\operatorname{Lip}(\widetilde{S}, \mathrm{~d}), X)} \leq 1$ such that $\tilde{f}_{S}\left(u_{i}^{\prime}\right) \in F\left(u_{i}^{\prime}\right)$ and $\tilde{f}_{S}\left(u_{i}^{\prime \prime}\right) \in F\left(u_{i}^{\prime \prime}\right), i=1,2$. One can readily see that the point $\tilde{f}_{S}\left(u_{1}\right) \in W_{1} \cap W_{2} \cap W_{3}$ proving the required property 3.25).

The proof of the proposition is complete.

We turn to the proof of inequality (1.8).

Note that, thanks to formula (3.9), for every $x, y \in \mathcal{M}$ we have

$$
F^{[2]}(x)=\bigcap_{u, u^{\prime}, u^{\prime \prime} \in \mathcal{M}} T_{x}\left(u, u^{\prime}, u^{\prime \prime}\right) \text { and } F^{[2]}(y)=\bigcap_{u, u^{\prime}, u^{\prime \prime} \in \mathcal{M}} T_{y}\left(u, u^{\prime}, u^{\prime \prime}\right) .
$$


Lemma 3.9. For every $\tau>0$ and every $x \in \mathcal{M}$ the following representation

$$
F^{[2]}(x)+\tau B_{X}=\bigcap\left\{\left[T_{x}\left(u, u^{\prime}, u^{\prime \prime}\right) \cap T_{x}\left(v, v^{\prime}, v^{\prime \prime}\right)\right]+\tau B_{X}\right\}
$$

holds. Here the first intersection in the right hand side of (3.27) is taken over all elements $u, u^{\prime}, u^{\prime \prime}, v, v^{\prime}, v^{\prime \prime} \in \mathcal{M}$.

Proof. The lemma is immediate from representation (3.26), Lemma 3.4 and Proposition 3.8 .

Proposition 3.10. For every $x, y \in \mathcal{M}$ the following inequality

$$
\mathrm{d}_{\mathrm{H}}\left(F^{[2]}(x), F^{[2]}(y)\right) \leq \gamma_{0}(L) \mathrm{d}(x, y)
$$

holds. Here $\gamma_{0}(L)=L \theta(L)^{2}$ where $\theta(L)$ is the constant from Theorem 2.3

Proof. Let $x, y \in \mathcal{M}$ and let $\tau=\gamma_{0}(L) \mathrm{d}(x, y)$. Prove that

$$
F^{[2]}(x)+\gamma_{0}(L) \mathrm{d}(x, y) B_{X}=F^{[2]}(x)+\tau B_{X} \supset F^{[2]}(y) .
$$

Lemma 3.9 tells us that this inclusion holds provided

$$
A=\left[T_{x}\left(u, u^{\prime}, u^{\prime \prime}\right) \cap T_{x}\left(v, v^{\prime}, v^{\prime \prime}\right)\right]+\tau B_{X} \supset F^{[2]}(y)
$$

for arbitrary $u, u^{\prime}, u^{\prime \prime}, v, v^{\prime}, v^{\prime \prime} \in \mathcal{M}$. We note that the set $A$ is the orbit of $y$ with respect to the diagram shown in Fig. 21. (I.e., the set $A$ consists of all points $a=g(y)$ where $g$ runs over all mappings which agree with the diagram in Fig. 21.)

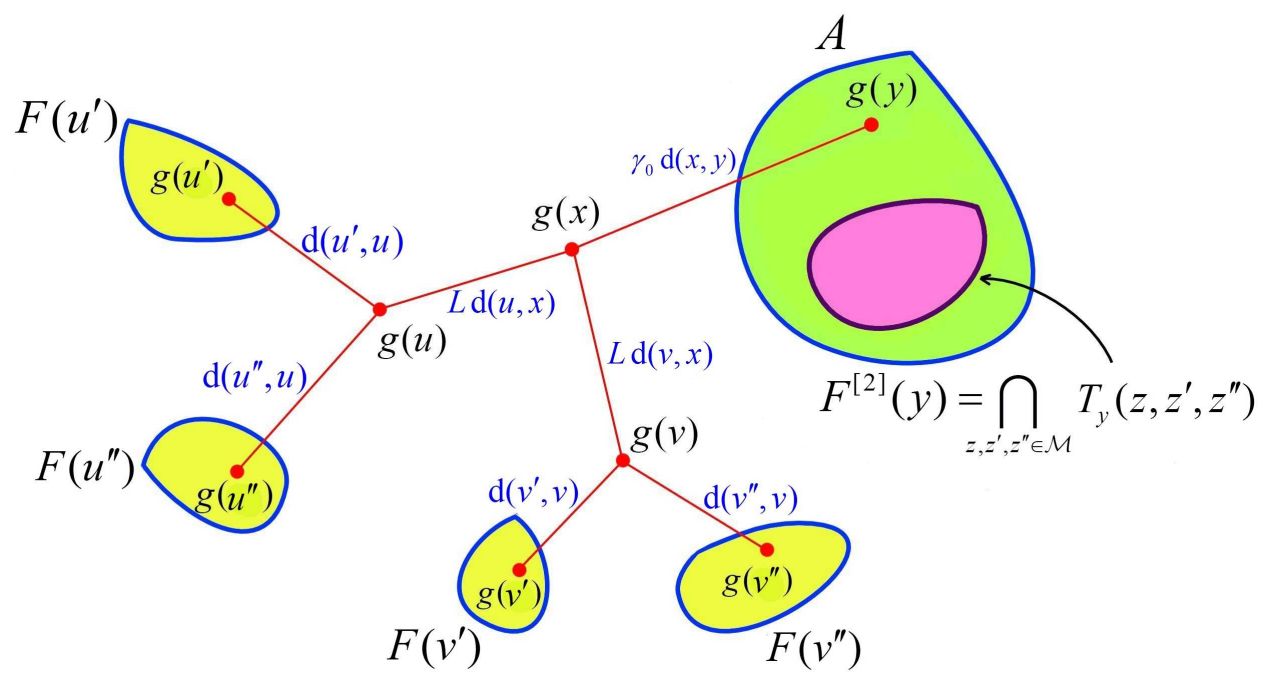

Fig. 21: The set $A$ is the orbit of $y$ with respect to this diagram.

In fact, we prove a stronger inclusion than 3.30 , namely that

$$
\begin{aligned}
A \supset & T_{y}\left(u, u^{\prime}, u^{\prime \prime}\right) \bigcap T_{y}\left(v, v^{\prime}, v^{\prime \prime}\right) \bigcap T_{y}\left(x, u^{\prime}, v^{\prime}\right) \bigcap \\
& T_{y}\left(x, u^{\prime}, v^{\prime \prime}\right) \bigcap T_{y}\left(x, u^{\prime \prime}, v^{\prime}\right) \bigcap T_{y}\left(x, u^{\prime \prime}, v^{\prime \prime}\right) .
\end{aligned}
$$


To prove the above inclusion, we introduce the following sets:

$$
C_{1}=F\left(u^{\prime}\right)+\mathrm{d}\left(u^{\prime}, u\right) B_{X}, \quad C_{2}=F\left(u^{\prime \prime}\right)+\mathrm{d}\left(u^{\prime \prime}, u\right) B_{X}, \quad C=T_{x}\left(v, v^{\prime}, v^{\prime \prime}\right) .
$$

Let

$$
\varepsilon=L \theta(L) \mathrm{d}(x, y) \quad \text { and } \quad r=\mathrm{d}(x, u) .
$$

Then $\tau=\gamma_{0}(L) \mathrm{d}(x, y)=\theta(L) \varepsilon$, and

$$
A=\left[T_{x}\left(u, u^{\prime}, u^{\prime \prime}\right) \cap T_{x}\left(v, v^{\prime}, v^{\prime \prime}\right)\right]+\tau B_{X}=\left[\left(C_{1} \cap C_{2}\right)+L r B_{X}\right] \cap C+\theta(L) \varepsilon B_{X} .
$$

See Fig. 22.

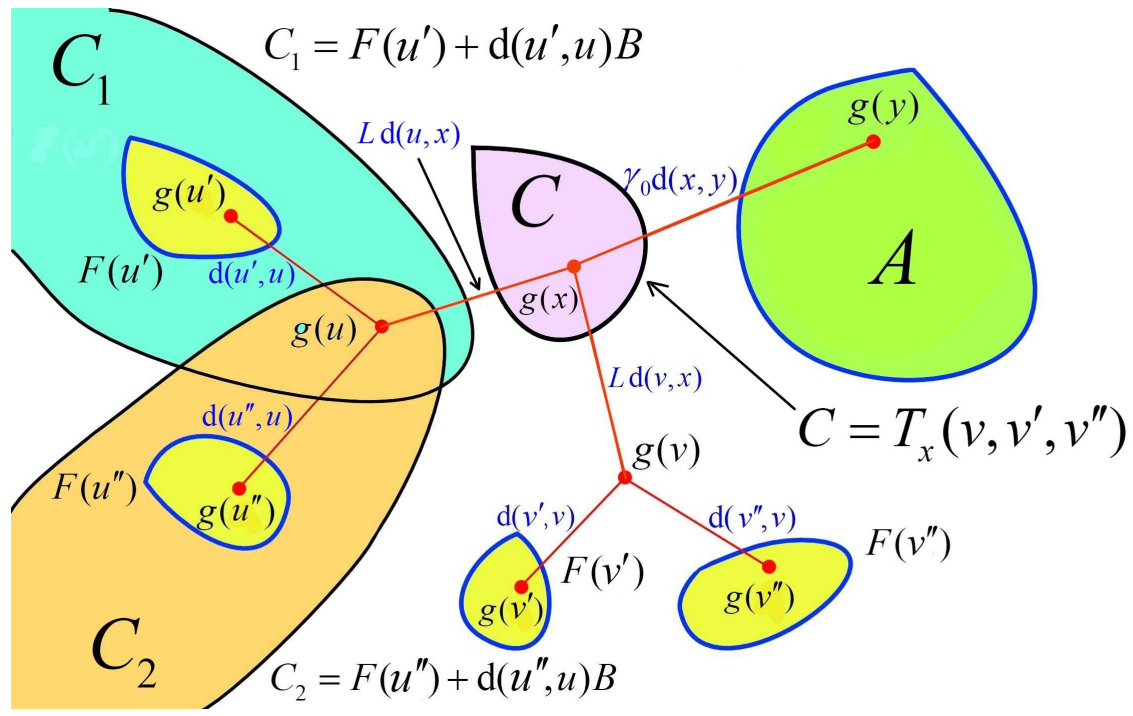

Fig. 22: The sets $C, C_{1}$ and $C_{2}$.

We want to apply Proposition 2.6 to the set $A$. To do this, we have to verify condition (2.22) of this proposition, i.e., to show that

$$
C_{1} \cap C_{2} \cap\left(C+r B_{X}\right) \neq \emptyset \text {. }
$$

Let $S=\left\{u^{\prime}, u^{\prime \prime}, v^{\prime}, v^{\prime \prime}\right\}$ and let $\widetilde{S}=\left\{x, u, u^{\prime}, u^{\prime \prime}, v, v^{\prime}, v^{\prime \prime}\right\}$. Claim 3.3 tells us that there exists a d-Lipschitz mapping $\tilde{f}_{S}: \widetilde{S} \rightarrow X$ with d-Lipschitz seminorm $\left\|\tilde{f}_{S}\right\|_{\operatorname{Lip}(\widetilde{S}, \mathrm{~d}), X)} \leq 1$ such that

$$
\tilde{f}_{S}\left(u^{\prime}\right) \in F\left(u^{\prime}\right), \quad \tilde{f}_{S}\left(u^{\prime \prime}\right) \in F\left(u^{\prime \prime}\right), \quad \tilde{f}_{S}\left(v^{\prime}\right) \in F\left(v^{\prime}\right) \text { and } \tilde{f}_{S}\left(v^{\prime \prime}\right) \in F\left(v^{\prime \prime}\right) .
$$

Prove that $\tilde{f}_{S}(u)$ belongs to the left hand side of 3.33 . Indeed, thanks to the inequality $\left\|\tilde{f}_{S}\right\|_{\operatorname{Lip}(\widetilde{S}, \mathrm{~d}), X)} \leq 1$, we have

$\left\|\tilde{f}_{S}\left(u^{\prime}\right)-\tilde{f}_{S}(u)\right\| \leq \mathrm{d}\left(u^{\prime}, u\right), \quad\left\|\tilde{f}_{S}\left(u^{\prime \prime}\right)-\tilde{f}_{S}(u)\right\| \leq \mathrm{d}\left(u^{\prime \prime}, u\right) \quad\left\|\tilde{f}_{S}(x)-\tilde{f}_{S}(u)\right\| \leq \mathrm{d}(x, u)=r$. 
and $\left\|\tilde{f}_{S}(x)-\tilde{f}_{S}(u)\right\| \leq \mathrm{d}(x, u)=r$. Thanks to these properties and 3.31$), \tilde{f}_{S}(u) \in C_{1} \cap C_{2}$.

In a similar way we show that $\tilde{f}_{S}(x) \in T_{x}\left(v, v^{\prime}, v^{\prime \prime}\right)=C$, see 3.31) and 3.8). Hence, we have $\tilde{f}_{S}(u) \in C+r B_{X}$. Thus,

$$
C_{1} \cap C_{2} \cap\left(C+r B_{X}\right) \ni \tilde{f}_{S}(u)
$$

proving 3.33).

We see that property (2.22) of Proposition 2.6 holds. This proposition tells us that

$$
\begin{aligned}
A & =\left[\left(C_{1} \cap C_{2}\right)+L r B_{X}\right] \cap C+\theta(L) \varepsilon B_{X} \\
& \supset\left[\left(C_{1} \cap C_{2}\right)+(L r+\varepsilon) B_{X}\right] \cap\left\{\left[\left(C_{1}+r B_{X}\right) \cap C\right]+\varepsilon B_{X}\right\} \cap\left\{\left[\left(C_{2}+r B_{X}\right) \cap C\right]+\varepsilon B_{X}\right\} \\
& =A_{1} \cap A_{2} \cap A_{3} .
\end{aligned}
$$

Prove that

$$
A_{i} \supset F^{[2]}(y) \text { for every } i=1,2,3 \text {. }
$$

We begin with the set

$$
A_{1}=\left[C_{1} \cap C_{2}\right]+(L r+\varepsilon) B_{X}
$$

Thus,

$$
A_{1}=\left[\left(F\left(u^{\prime}\right)+\mathrm{d}\left(u^{\prime}, u\right) B_{X}\right) \bigcap\left(F\left(u^{\prime \prime}\right)+\mathrm{d}\left(u^{\prime \prime}, u\right) B_{X}\right)\right]+(L \mathrm{~d}(u, x)+L \theta(L) \mathrm{d}(x, y)) B_{X} .
$$

See (3.31). By the triangle inequality,

$$
\mathrm{d}(u, x)+\theta(L) \mathrm{d}(x, y) \geq \mathrm{d}(u, x)+\mathrm{d}(x, y) \geq \mathrm{d}(u, y) .
$$

Hence,

$$
A_{1} \supset\left[\left(F\left(u^{\prime}\right)+\mathrm{d}\left(u^{\prime}, u\right) B_{X}\right) \bigcap\left(F\left(u^{\prime \prime}\right)+\mathrm{d}\left(u^{\prime \prime}, u\right) B_{X}\right)\right]+L \mathrm{~d}(u, y) B_{X}=T_{y}\left(u, u^{\prime}, u^{\prime \prime}\right) .
$$

But $T_{y}\left(u, u^{\prime}, u^{\prime \prime}\right) \supset F^{[2]}(y)$, see 3.26 , so that $A_{1} \supset F^{[2]}(y)$.

We turn to the proof of the inclusion $A_{2} \supset F^{[2]}(y)$. Note that $A_{2}$ is defined by

$$
A_{2}=\left[\left(C_{1}+r B_{X}\right) \cap C\right]+\varepsilon B_{X}
$$

By the triangle inequality,

$$
C_{1}+r B_{X}=F\left(u^{\prime}\right)+\mathrm{d}\left(u^{\prime}, u\right) B_{X}+\mathrm{d}(u, x) B_{X} \supset F\left(u^{\prime}\right)+\mathrm{d}\left(u^{\prime}, x\right) B_{X} .
$$

Let

$$
\widetilde{C}=F\left(u^{\prime}\right)+\mathrm{d}\left(u^{\prime}, x\right) B_{X}, \quad \widetilde{C}_{1}=F\left(v^{\prime}\right)+\mathrm{d}\left(v^{\prime}, v\right) B_{X}, \quad \widetilde{C}_{2}=F\left(v^{\prime \prime}\right)+\mathrm{d}\left(v^{\prime \prime}, v\right) B_{X},
$$


and let

$$
\tilde{r}=\mathrm{d}(v, x) .
$$

In these settings

$$
C=T_{x}\left(v, v^{\prime}, v^{\prime \prime}\right)=\left[\widetilde{C}_{1} \cap \widetilde{C}_{2}\right]+L \tilde{r} B_{X}
$$

Let

$$
\widetilde{A}=\left\{\left[\left(\widetilde{C}_{1} \cap \widetilde{C}_{2}\right)+L \tilde{r} B_{X}\right] \cap \widetilde{C}\right\}+\varepsilon B_{X}
$$

Then, thanks to 3.35) and 3.36,

$$
A_{2} \supset\left[\left(F\left(u^{\prime}\right)+\mathrm{d}\left(u^{\prime}, x\right) B_{X}\right) \cap C\right]+\varepsilon B_{X}=\left\{\left[\left(\widetilde{C}_{1} \cap \widetilde{C}_{2}\right)+L \tilde{r} B_{X}\right] \cap \widetilde{C}\right\}+\varepsilon B_{X}=\widetilde{A} .
$$

See Fig. 23.

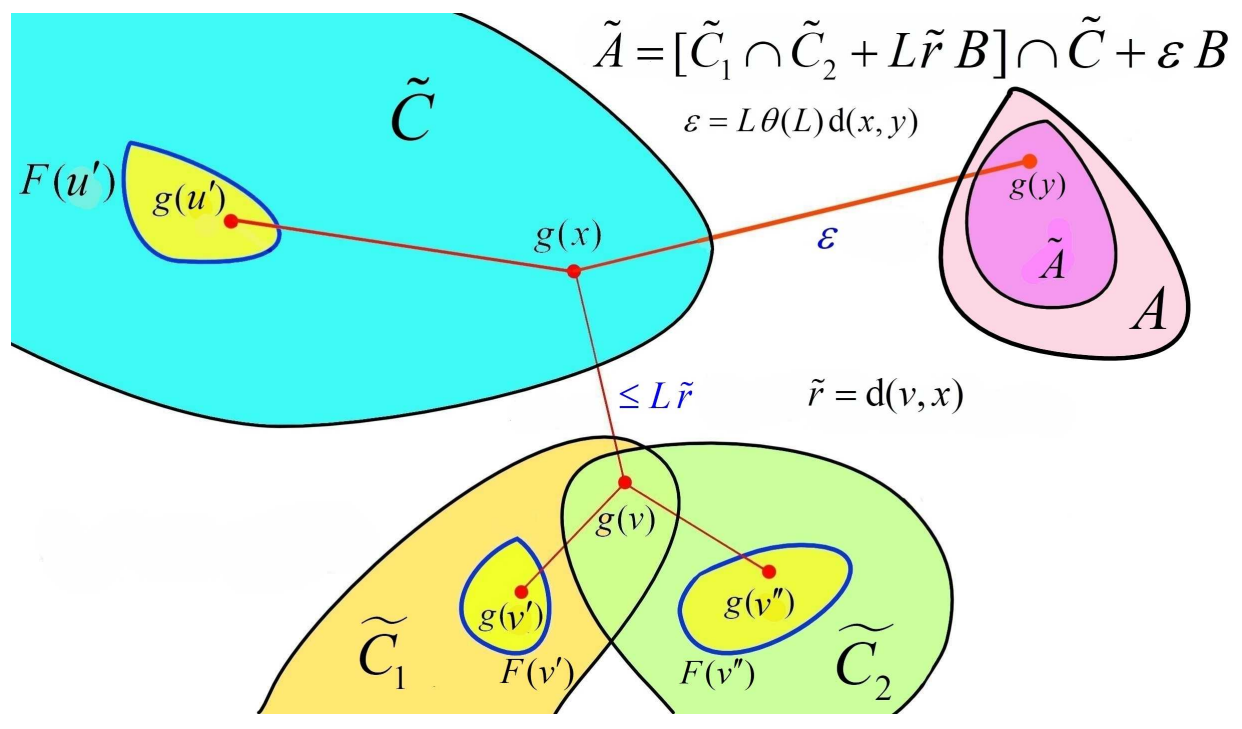

Fig. 23: The sets $\widetilde{C}, \widetilde{C}_{1}$ and $\widetilde{C}_{2}$.

Prove that $\widetilde{A} \supset F^{[2]}(y)$. We will do this by applying Proposition 2.6 to the set $\widetilde{A}$. But first we must check the hypothesis of this proposition, i.e., to show that

$$
\widetilde{C}_{1} \cap \widetilde{C}_{2} \cap\left(\widetilde{C}+\tilde{r} B_{X}\right) \neq \emptyset
$$

To establish this property, we set $S=\left\{u^{\prime}, v^{\prime}, v^{\prime \prime}\right\}$ and $\widetilde{S}=\left\{x, u^{\prime}, v, v^{\prime}, v^{\prime \prime}\right\}$. Claim 3.3 tells us that there exists a d-Lipschitz mapping $\tilde{f}_{S}: \widetilde{S} \rightarrow X$ with d-Lipschitz seminorm $\left\|\tilde{f}_{S}\right\|_{\operatorname{Lip}((\widetilde{S}, \mathrm{~d}), X)} \leq 1$ such that

$$
\tilde{f}_{S}\left(u^{\prime}\right) \in F\left(u^{\prime}\right), \quad \tilde{f}_{S}\left(v^{\prime}\right) \in F\left(v^{\prime}\right) \text { and } \tilde{f}_{S}\left(v^{\prime \prime}\right) \in F\left(v^{\prime \prime}\right)
$$

Combining these properties of $\tilde{f}_{S}$ with definitions 3.37 and 3.38 , we conclude that $\widetilde{C}_{1} \cap \widetilde{C}_{2} \cap\left(\widetilde{C}+\tilde{r} B_{X}\right) \ni \tilde{f}_{S}(v)$ proving 3.40). 
We recall that $\varepsilon=L \theta(L) \mathrm{d}(x, y)$, see 3.32, so that

$$
\left.\left.\widetilde{A}=\left[\left\{\left(\widetilde{C}_{1} \cap \widetilde{C}_{2}\right)+L \tilde{r} B_{X}\right\} \cap \widetilde{C}\right]+L \theta(L) \mathrm{d}(x, y) B_{X}, \quad \text { (see } 3.39\right)\right) .
$$

We apply Proposition 2.6 to $\widetilde{A}$ and obtain the following:

$$
\begin{aligned}
\widetilde{A} & \supset\left\{\left(\widetilde{C}_{1} \cap \widetilde{C}_{2}\right)+(L \tilde{r}+L \mathrm{~d}(x, y)) B_{X}\right\} \\
& \cap\left\{\left[\left(\widetilde{C}_{1}+\tilde{r} B_{X}\right) \cap \widetilde{C}\right]+L \mathrm{~d}(x, y) B_{X}\right\} \cap\left\{\left[\left(\widetilde{C}_{2}+\tilde{r} B_{X}\right) \cap \widetilde{C}\right]+L \mathrm{~d}(x, y) B_{X}\right\} \\
& =\widetilde{A_{1}} \cap \widetilde{A_{2}} \cap \widetilde{A_{3}} .
\end{aligned}
$$

Prove that $\widetilde{A_{i}} \supset F^{[2]}(y)$ for every $i=1,2,3$. First, let us show that

$$
\widetilde{A_{1}}=\left(\widetilde{C}_{1} \cap \widetilde{C}_{2}\right)+(L \tilde{r}+L \mathrm{~d}(x, y)) B_{X} \supset F^{[2]}(y) .
$$

By (3.38) and the triangle inequality, $\tilde{r}+\mathrm{d}(x, y)=\mathrm{d}(v, x)+\mathrm{d}(x, y) \geq \mathrm{d}(v, y)$ so that

$$
\begin{aligned}
\widetilde{A_{1}} & \supset\left(\widetilde{C}_{1} \cap \widetilde{C}_{2}\right)+L \mathrm{~d}(v, y) B_{X} \\
& =\left[\left(F\left(v^{\prime}\right)+\mathrm{d}\left(v^{\prime}, v\right) B_{X}\right) \cap\left(F\left(v^{\prime \prime}\right)+\mathrm{d}\left(v^{\prime \prime}, v\right) B_{X}\right)\right]+L \mathrm{~d}(v, y) B_{X} \\
& =T_{y}\left(v, v^{\prime}, v^{\prime \prime}\right) .
\end{aligned}
$$

See (3.37) and (3.8). This inclusion and (3.26) imply (3.41).

Next, let us show that

$$
\left.\widetilde{A_{2}}=\left[\left(\widetilde{C}_{1}+\tilde{r} B_{X}\right) \cap \widetilde{C}\right]+L \mathrm{~d}(x, y) B_{X} \supset F^{[2]}(y)\right) .
$$

Thanks to 3.37, 3.38 and the triangle inequality,

$$
\widetilde{C}_{1}+\tilde{r} B_{X}=F\left(v^{\prime}\right)+\mathrm{d}\left(v^{\prime}, v\right) B_{X}+\mathrm{d}(v, x) B_{X} \supset F\left(v^{\prime}\right)+\mathrm{d}\left(v^{\prime}, x\right) B_{X}
$$

so that

$$
\widetilde{A_{2}} \supset\left[\left(F\left(v^{\prime}\right)+\mathrm{d}\left(v^{\prime}, x\right) B_{X}\right) \bigcap\left(F\left(u^{\prime}\right)+\mathrm{d}\left(u^{\prime}, x\right) B_{X}\right)\right]+L \mathrm{~d}(x, y) B_{X}=T_{y}\left(x, u^{\prime}, v^{\prime}\right) .
$$

See 3.8. From this and 3.26, we have $\widetilde{A_{2}} \supset T_{y}\left(x, u^{\prime}, v^{\prime}\right) \supset F^{[2]}(y)$, proving 3.42.

In the same way we show that

$$
\widetilde{A_{3}}=\left[\left(\widetilde{C}_{2}+\tilde{r} B_{X}\right) \cap \widetilde{C}\right]+L \mathrm{~d}(x, y) B_{X} \supset T_{y}\left(x, u^{\prime}, v^{\prime \prime}\right) \supset F^{[2]}(y) .
$$

Combining this with 3.41 and 3.42, we obtain the required inclusion $\widetilde{A_{i}} \supset F^{[2]}(y)$ for every $i=1,2,3$. In turn, this proves that

$$
\widetilde{A} \supset F^{[2]}(y) \quad \text { because } \widetilde{A} \supset \widetilde{A_{1}} \cap \widetilde{A_{2}} \cap \widetilde{A_{3}} \supset F^{[2]}(y)
$$

We know that $A_{2} \supset \widetilde{A}$, so that $A_{2} \supset F^{[2]}(y)$. In the same fashion we show that

$$
A_{3}=\left[\left(C_{2}+r B_{X}\right) \cap C\right]+L \varepsilon B_{X} \supset F^{[2]}(y)
$$


proving 3.34). Hence, $A \supset A_{1} \cap A_{2} \cap A_{3} \supset F^{[2]}(y)$ so that 3.30 holds.

Thus, (3.29) is proved. By interchanging the roles of elements $x$ and $y$ in this inclusion we obtain the inclusion $F^{[2]}(y)+\gamma_{0}(L) \mathrm{d}(x, y) B_{X} \supset F^{[2]}(x)$. These two inclusions imply inequality 3.28 proving the proposition.

We are in a position to complete the proof of Theorem 1.9 .

Recall that $\lambda_{1}, \lambda_{2}$ are parameters satisfying (1.5), and $L=\lambda_{2} / \lambda_{1}$. Thus, $L$ and $\lambda_{1}$ satisfy (3.1). We also recall that $\mathrm{d}=\lambda_{1} \rho$, see (3.2). Let $\gamma$ be a parameter satisfying (1.5).

In these settings, the mappings $F^{[1]}$ and $F^{[2]}$ defined by formulae (3.3) and (3.4) are the first and the second order $\left(\left\{\lambda_{1}, \lambda_{2}\right\}, \rho\right)$-balanced refinements of $F$ respectively. See Definition 1.6.

Proposition 3.8 tells us that, under these conditions, $F^{[2]}(x) \neq \emptyset$ on $\mathcal{M}$. In turn, Proposition 3.10 states that

$$
\mathrm{d}_{\mathrm{H}}\left(F^{[2]}(x), F^{[2]}(y)\right) \leq \gamma_{0}(L) \mathrm{d}(x, y) \quad \text { for all } \quad x, y \in \mathcal{M} .
$$

Recall that $\gamma_{0}(L)=L \cdot \theta(L)^{2}$ where $\theta=\theta(L)=(3 L+1) /(L-1)$, see 2.8). Hence, $\theta(L)=\left(3 \lambda_{2}+\lambda_{1}\right) /\left(\lambda_{2}-\lambda_{1}\right)$ so that

$$
\left.\mathrm{d}_{\mathrm{H}}\left(F^{[2]}(x), F^{[2]}(y)\right) \leq \gamma_{0}(L) \mathrm{d}(x, y) \leq L \cdot \theta(L)^{2} \mathrm{~d}(x, y)\right)=\lambda_{2} \frac{\left(3 \lambda_{2}+\lambda_{1}\right)^{2}}{\left(\lambda_{2}-\lambda_{1}\right)^{2}} \rho(x, y) .
$$

Combining this inequality with the third inequality in $(1.5)$, we obtain $(1.8)$ proving Theorem 1.9 for the parameters $\lambda_{1}, \lambda_{2}$ and $\gamma$ satisfying (1.5).

In particular, one can set $\lambda_{1}=4 / 3, \lambda_{2}=4$, and $\gamma=100$. Indeed, in this case $e(\mathfrak{M}, X) \leq \lambda_{1}=4 / 3$, see Remark 3.2 , so that $\lambda_{1}, \lambda_{2}$ and $\gamma$ satisfy 1.5 .

Next, let $X$ be a two dimensional Euclidean space, and let $\lambda_{1}, \lambda_{2}$ and $\gamma$ satisfy (1.9). In this case, we prove $(1.7)$ and $(1.8)$ by replacing in the proof of Theorem 1.9 the function $\theta=\theta(L)$ defined by 2.8 with the function $\theta(L)=1+2 L / \sqrt{L^{2}-1}$. We leave the details to the interested reader.

In particular, we can set $\lambda_{1}=4 / \pi, \lambda_{2}=12 / \pi, \gamma=38$. Indeed, in this case, $e(\mathfrak{M}, X) \leq$ $4 / \pi$, see Remark 3.2, which implies (1.9) for these values of parameters $\lambda_{1}, \lambda_{2}$ and $\gamma$.

Finally, suppose that $X$ is a Euclidean space, $\mathcal{M}$ is a subset of a Euclidean space $E$, and $\rho$ is the Euclidean metric in $E$. In this case $e(\mathfrak{M}, X)=1$, see Remark 3.2, so that one can set $\lambda_{1}=1, \lambda_{2}=3$ and $\gamma=25$. Clearly, in this case inequalities (1.9) hold.

The proof of Theorem 1.9 is complete.

\section{Balanced refinements of line segments in a Banach space.}

In this section we prove Theorem 1.10

Let $(\mathcal{M}, \rho)$ be a pseudometric space, and let $(X,\|\cdot\|)$ be a Banach space. We assume that $\operatorname{dim} X>1$. Let us recall that $\mathcal{K}_{1}(X)$ is the family of all non-empty convex compacts in $X$ of dimension at most 1 (i.e., the family of all points and all bounded closed line segments in $X$ ).

We need the following version of one dimensional Helly's Theorem. 
Theorem 4.1. Let $\mathcal{K}$ be a collection of closed convex subsets of $X$ containing a set $K_{0} \in \mathcal{K}_{1}(X)$. If $K_{0}$ has a common point with any two members of $\mathcal{K}$, then there exists a point common to all of the collection $\mathcal{K}$.

Proof. We introduce a family $\widetilde{\mathcal{K}}=\left\{K \cap K_{0}: K \in \mathcal{K}\right\}$, and apply to $\widetilde{\mathcal{K}}$ one dimensional Helly's Theorem. (See part (a) of Lemma 5.2.)

We also need the following variant of Proposition 2.6 for the family $\mathcal{K}_{1}(X)$.

Proposition 4.2. Let $X$ be a Banach space, and let $r \geq 0$. Let $C, C_{1}, C_{2} \subset X$ be convex closed subsets, and let $C_{1} \in \mathcal{K}_{1}(X)$. Suppose that

$$
C_{1} \cap C_{2} \cap\left(C+r B_{X}\right) \neq \emptyset .
$$

Then for every $L>1$ and every $\varepsilon>0$ the following inclusion

$\left[\left\{\left(C_{1} \cap C_{2}\right)+L r B_{X}\right\} \cap C\right]+\theta(L) \varepsilon B_{X} \supset\left[\left(C_{1} \cap C_{2}\right)+(L r+\varepsilon) B_{X}\right] \cap\left[\left\{\left(C_{1}+r B_{X}\right) \cap C\right\}+\varepsilon B_{X}\right]$

holds. Here $\theta(L)$ is the same as in Theorem 2.3 i.e., $\theta(L)=(3 L+1) /(L-1)$ for an arbitrary $X$, and $\theta(L)=1+2 L / \sqrt{L^{2}-1}$ whenever $X$ is a Euclidean space.

Proof. Let

$$
a \in\left[\left(C_{1} \cap C_{2}\right)+(L r+\varepsilon) B_{X}\right] \cap\left\{\left[\left(C_{1}+r B_{X}\right) \cap C\right]+\varepsilon B_{X}\right\} .
$$

Prove that

$$
a \in\left\{\left[\left(C_{1} \cap C_{2}\right)+L r B_{X}\right] \cap C\right\}+\theta(L) \varepsilon B_{X} .
$$

First, let us show that

$$
C_{1} \cap C_{2} \cap\left(C+r B_{X}\right) \cap B_{X}(a, L r+\varepsilon) \neq \emptyset .
$$

Recall that $C_{1} \in \mathcal{K}_{1}(X)$. Helly's Theorem 4.1 tells us that it is suffices to show that any two sets in the left hand size of (4.4) have a common point with $C_{1}$.

First we note that $C_{1}, C_{2}$ and $C+r B_{X}$ have a common point. See (4.1). We also know that

$$
a \in\left(C_{1} \cap C_{2}\right)+(L r+\varepsilon) B_{X},
$$

see (4.2), so that $C_{1} \cap C_{2} \cap B_{X}(a, L r+\varepsilon) \neq \emptyset$.

Let us prove that

$$
C_{1} \cap\left(C+r B_{X}\right) \cap B_{X}(a, L r+\varepsilon) \neq \emptyset .
$$

Property (4.2) tells us that $a \in\left[\left(C_{1}+r B_{X}\right) \cap C\right]+\varepsilon B_{X}$. Let $b \in X$ be a point nearest to $a$ on $\left(C_{1}+r B_{X}\right) \cap C$, and let $b_{1} \in X$ be a point nearest to $b$ on $C_{1}$. Then $\left\|b_{1}-b\right\| \leq r$ and $\|a-b\| \leq \varepsilon$. Moreover, $b_{1} \in C_{1} \cap\left(C+r B_{X}\right)$ and

$$
\left\|a-b_{1}\right\| \leq\|a-b\|+\left\|b-b_{1}\right\| \leq \varepsilon+r \leq \varepsilon+L r,
$$

so that $b_{1} \in B_{X}(a, L r+\varepsilon)$. Hence,

$$
b_{1} \in C_{1} \cap\left(C+r B_{X}\right) \cap B_{X}(a, L r+\varepsilon)
$$


proving (4.5).

Thus, (4.4) holds proving the existence of a point $x \in X$ such that

$$
x \in C_{1} \cap C_{2} \cap\left(C+r B_{X}\right) \cap B_{X}(a, L r+\varepsilon) .
$$

In particular, $x \in C+r B_{X}$ so that $B_{X}(x, r) \cap C \neq \emptyset$ proving that condition (2.6) of Proposition 2.3 is satisfied. We apply this proposition to $x, r$ and the set $C$ and get:

$\left[C \cap B_{X}(x, L r)\right]+\theta(L) \varepsilon B_{X} \supset\left(C+\varepsilon B_{X}\right) \cap\left(B_{X}(x, L r)+\varepsilon B_{X}\right)=\left(C+\varepsilon B_{X}\right) \cap B_{X}(x, L r+\varepsilon)$.

From (4.6) we learn that $a \in B_{X}(x, L r+\varepsilon)$. In turn, (4.2) tells us that

$$
a \in\left[\left(C_{1}+r B_{X}\right) \cap C\right]+\varepsilon B_{X} \subset C+\varepsilon B_{X} .
$$

Hence, $\left(C+\varepsilon B_{X}\right) \cap B_{X}(x, L r+\varepsilon) \ni a$ proving that $\left[C \cap B_{X}(x, L r)\right]+\theta(L) \varepsilon B_{X} \ni a$.

Finally, (4.6) tells us that $x \in C_{1} \cap C_{2}$ proving the required inclusion (4.3).

We recall that $\operatorname{dim} X>1$ so that the finiteness number $N(1, X)=\min \left\{2^{2}, 2^{\operatorname{dim} X}\right\}=4$. Let $F: \mathcal{M} \rightarrow \mathcal{K}_{1}(X)$ be a set-valued mapping. We suppose that $F$ satisfies the hypothesis of Theorem 1.10 , i.e., the following statement is true.

Claim 4.3. For every subset $\mathcal{M}^{\prime} \subset \mathcal{M}$ with $\# \mathcal{M}^{\prime} \leq 4$ the restriction $\left.F\right|_{\mathcal{M}^{\prime}}$ of $F$ to $\mathcal{M}^{\prime}$ has a Lipschitz selection $f_{\mathcal{M}^{\prime}}: \mathcal{M}^{\prime} \rightarrow X$ with $\|f\|_{\operatorname{Lip}\left(\mathcal{M}^{\prime}, X\right)} \leq 1$.

Let $\vec{\lambda}=\left\{\lambda_{1}, \lambda_{2}\right\}$, and let $F^{[1]}$ and $F^{[2]}$ be the first and the second order $(\vec{\lambda}, \rho)$-balanced refinements of $F$. See Definition 1.6, Our aim is to show that if

$$
\lambda_{1} \geq 1, \quad \lambda_{2} \geq 3 \lambda_{1}, \quad \gamma \geq \lambda_{2}\left(3 \lambda_{2}+\lambda_{1}\right) /\left(\lambda_{2}-\lambda_{1}\right),
$$

then the set-valued mapping $F^{[2]}$ is a $\gamma$-core of $F$ (with respect to $\rho$ ), i.e.,

$$
F^{[2]}(x) \neq \emptyset \text { for every } x \in \mathcal{M} \text {, and } \mathrm{d}_{\mathrm{H}}\left(F^{[2]}(x), F^{[2]}(y)\right) \leq \gamma \rho(x, y) \text { for all } x, y \in \mathcal{M} \text {. }
$$

To prove this, we set $L=\lambda_{2} / \lambda_{1}$ and introduce a new pseudometric on $\mathcal{M}$ defined by

$$
\mathrm{d}(x, y)=\lambda_{1} \rho(x, y), \quad x, y \in \mathcal{M} .
$$

Note that, thanks to 4.77,

$$
L \geq 3 \quad \text { and } \quad \rho \leq \mathrm{d} \quad \text { on } \mathcal{M} .
$$

We also note that in these settings, for every $x \in \mathcal{M}$,

$$
F^{[1]}(x)=\bigcap_{z \in \mathcal{M}}\left[F(z)+\mathrm{d}(x, z) B_{X}\right] \text { and } F^{[2]}(x)=\bigcap_{z \in \mathcal{M}}\left[F^{[1]}(z)+L \mathrm{~d}(x, z) B_{X}\right] .
$$

Next, we need the following analog of Lemma 3.4 .

Lemma 4.4. Let $\mathcal{K}$ be a collection of convex closed subsets of $X$ containing a set $K_{0} \in$ $\mathcal{K}_{1}(X)$. Suppose that $\cap\{K: K \in \mathcal{K}\} \neq \emptyset$. Then for every $r \geq 0$, we have

$$
\left(\bigcap_{K \in \mathcal{K}} K\right)+r B_{X}=\bigcap_{K \in \mathcal{K}}\left\{\left[K \cap K_{0}\right]+r B_{X}\right\} .
$$


Proof. Let $\widetilde{\mathcal{K}}=\left\{K \cap K_{0}: K \in \mathcal{K}\right\}$. Clearly, $\widetilde{\mathcal{K}} \subset \mathcal{K}_{1}(X)$. It is also clear that the statement of the lemma is equivalent to the equality

$$
\left(\bigcap_{\widetilde{K} \in \widetilde{\mathcal{K}}} \widetilde{K}\right)+r B_{X}=\bigcap_{\widetilde{K} \in \widetilde{\mathcal{K}}}\left\{\widetilde{K}+r B_{X}\right\}
$$

provided $\cap\{\widetilde{K}: \widetilde{K} \in \mathcal{K}\} \neq \emptyset$. We prove this equality by a slight modification of the proof of Lemma 3.4. More specifically, we obtain the result by using in that proof Helly's Theorem 4.1 instead of Theorem 2.5. We leave the details to the interested reader.

Lemma 4.5. For every $x \in \mathcal{M}$ the set $F^{[1]}(x)$ belongs to the family $\mathcal{K}_{1}(X)$. Moreover, for every $x, z \in \mathcal{M}$, we have

$$
F^{[1]}(z)+L \mathrm{~d}(x, z) B_{X}=\bigcap_{v \in \mathcal{M}}\left\{\left[\left(F(v)+\mathrm{d}(z, v) B_{X}\right) \cap F(z)\right]+L \mathrm{~d}(x, z) B_{X}\right\} .
$$

Proof. Let $\mathcal{K}=\left\{F(z)+\mathrm{d}(z, x) B_{X}: z \in \mathcal{M}\right\}$. Clearly, $\mathcal{K}$ is a family of all bounded closed convex subsets of $X$ containing the set $F(x) \in \mathcal{K}_{1}(X)$.

Theorem 4.1 tells us that the set $F^{[1]}(x)=\bigcap\{K: K \in \mathcal{K}\}$ is non-empty whenever for every $z^{\prime}, z^{\prime \prime} \in \mathcal{M}$ the set

$$
E\left(x, z^{\prime}, z^{\prime \prime}\right)=F(x) \bigcap\left[F\left(z^{\prime}\right)+\mathrm{d}\left(z^{\prime}, x\right) B_{X}\right] \cap\left[F\left(z^{\prime \prime}\right)+\mathrm{d}\left(z^{\prime \prime}, x\right) B_{X}\right] \neq \emptyset .
$$

Fix $z^{\prime}, z^{\prime \prime} \in \mathcal{M}$ and set $\mathcal{M}^{\prime}=\left\{x, z^{\prime}, z^{\prime \prime}\right\}$. Thanks to Claim 4.3, there exists a function $f_{\mathcal{M}^{\prime}}: \mathcal{M}^{\prime} \rightarrow X$ satisfying the following conditions: $f_{\mathcal{M}^{\prime}}(x) \in F(x), f_{\mathcal{M}^{\prime}}\left(z^{\prime}\right) \in F\left(z^{\prime}\right)$, $f_{\mathcal{M}^{\prime}}\left(z^{\prime \prime}\right) \in F\left(z^{\prime \prime}\right)$ and

$$
\left\|f_{\mathcal{M}^{\prime}}\left(z^{\prime}\right)-f_{\mathcal{M}^{\prime}}(x)\right\| \leq \rho\left(z^{\prime}, x\right) \leq \mathrm{d}\left(z^{\prime}, x\right), \quad\left\|f_{\mathcal{M}^{\prime}}\left(z^{\prime \prime}\right)-f_{\mathcal{M}^{\prime}}(x)\right\| \leq \rho\left(z^{\prime \prime}, x\right) \leq \mathrm{d}\left(z^{\prime \prime}, x\right) .
$$

See $(4.8)$. Then $f_{\mathcal{M}^{\prime}}(x) \in E\left(x, z^{\prime}, z^{\prime \prime}\right)$ so that 4.11 holds. Hence, $F^{[1]}(x) \neq \emptyset$ proving that $F^{[1]}(x) \in \mathcal{K}_{1}(X)$.

It remains to note that equality (4.10) is immediate from (4.9) and Lemma 4.4

The proof of the lemma is complete.

Lemma 4.6. For every $x \in \mathcal{M}$, the following equality

$$
F^{[2]}(x)=\bigcap_{u, u^{\prime} \in \mathcal{M}}\left\{\left[\left(F\left(u^{\prime}\right)+\mathrm{d}\left(u^{\prime}, u\right) B_{X}\right) \cap F(u)\right]+L \mathrm{~d}(u, x) B_{X}\right\}
$$

holds.

Proof. The lemma is immediate from representation 4.9 and Lemma 4.5.

Given $x, u, u^{\prime} \in \mathcal{M}$ we put

$$
\widetilde{T}_{x}\left(u, u^{\prime}\right)=\left[\left(F\left(u^{\prime}\right)+\mathrm{d}\left(u^{\prime}, u\right) B_{X}\right) \bigcap F(u)\right]+L \mathrm{~d}(u, x) B_{X} .
$$

Note that, in our terminology, the set $\widetilde{T}_{x}\left(u, u^{\prime}\right)$ is the orbit of $x$ with respect to the diagram shown in Fig. 24. Thus, $\widetilde{T}_{x}\left(u, u^{\prime}\right)$ is the family of all points $a=g(x)$ where $g$ runs over all mappings which agree with the diagram in Fig. 24 below. 


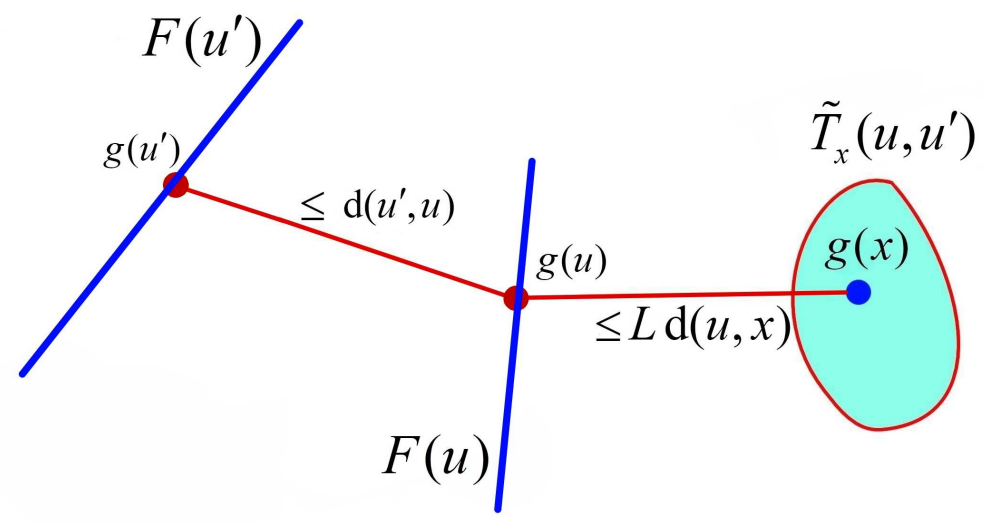

Fig. 24: $\widetilde{T}_{x}\left(u, u^{\prime}\right)$ is the orbit of $x$ with respect to this diagram.

Now, Lemma 4.6 reformulates as follows:

$$
F^{[2]}(x)=\bigcap_{u, u^{\prime} \in \mathcal{M}} \widetilde{T}_{x}\left(u, u^{\prime}\right)
$$

Proposition 4.7. For every $x \in \mathcal{M}$ the set $F^{[2]}(x)$ is non-empty.

Proof. Recall that $F(x) \in \mathcal{K}_{1}(X)$. Furthermore, by 4 .12), $F(x)=\widetilde{T}_{x}(x, x)$. Therefore, by (4.13) and Helly's Theorem 4.1, the set $F^{[2]}(x) \neq \emptyset$ whenever for every $u_{i}, u_{i}^{\prime} \in \mathcal{M}$, $i=1,2$, we have

$$
F(x) \cap \widetilde{T}_{x}\left(u_{1}, u_{1}^{\prime}\right) \cap \widetilde{T}_{x}\left(u_{2}, u_{2}^{\prime}\right) \neq \emptyset .
$$

We note that (4.14) holds provided there exists a mapping $g$ which agrees with the diagram shown in Fig. 25.

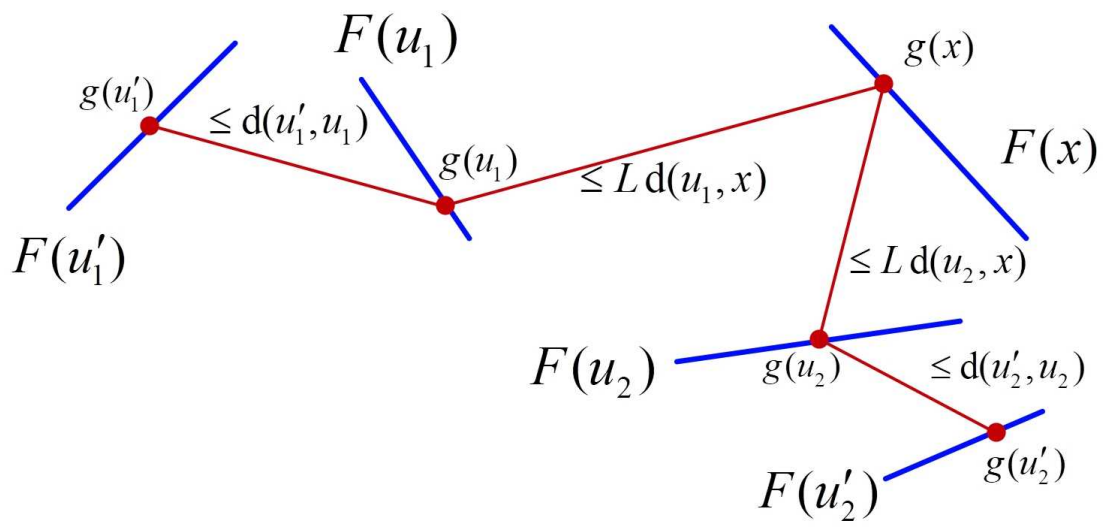

Fig. 25: The mapping $g$ agrees with this diagram. 
Thanks to 4.12,

$$
\widetilde{T}_{x}\left(u_{i}, u_{i}^{\prime}\right)=\left[\left(F\left(u_{i}^{\prime}\right)+\mathrm{d}\left(u_{i}^{\prime}, u_{i}\right) B_{X}\right) \cap F\left(u_{i}\right)\right]+L \mathrm{~d}\left(u_{i}, x\right) B_{X}, \quad i=1,2 .
$$

Fix elements $u_{1}, u_{1}^{\prime}, u_{2}, u_{2}^{\prime} \in \mathcal{M}$ and prove that property (4.14) holds.

First we note that, without loss of generality, one may assume that $\rho\left(u_{1}, x\right) \geq \rho\left(u_{2}, x\right)$. Next, we introduce the following sets:

$$
G_{1}=F\left(u_{2}\right), \quad G_{2}=F\left(u_{2}^{\prime}\right)+\rho\left(u_{2}, u_{2}^{\prime}\right) B_{X}, \quad G_{3}=F(x)+\rho\left(u_{2}, x\right) B_{X},
$$

and

$$
G_{4}=\left[\left(F\left(u_{1}^{\prime}\right)+\rho\left(u_{1}^{\prime}, u_{1}\right) B_{X}\right) \bigcap F\left(u_{1}\right)\right]+\rho\left(u_{1}, u_{2},\right) B_{X} .
$$

Prove that if

$$
G_{1} \cap G_{2} \cap G_{3} \cap G_{4} \neq \emptyset
$$

then (4.14) holds.

(Note that (4.18) holds whenever there exists a mapping $g$ which agrees with the diagram in Fig. 26.)

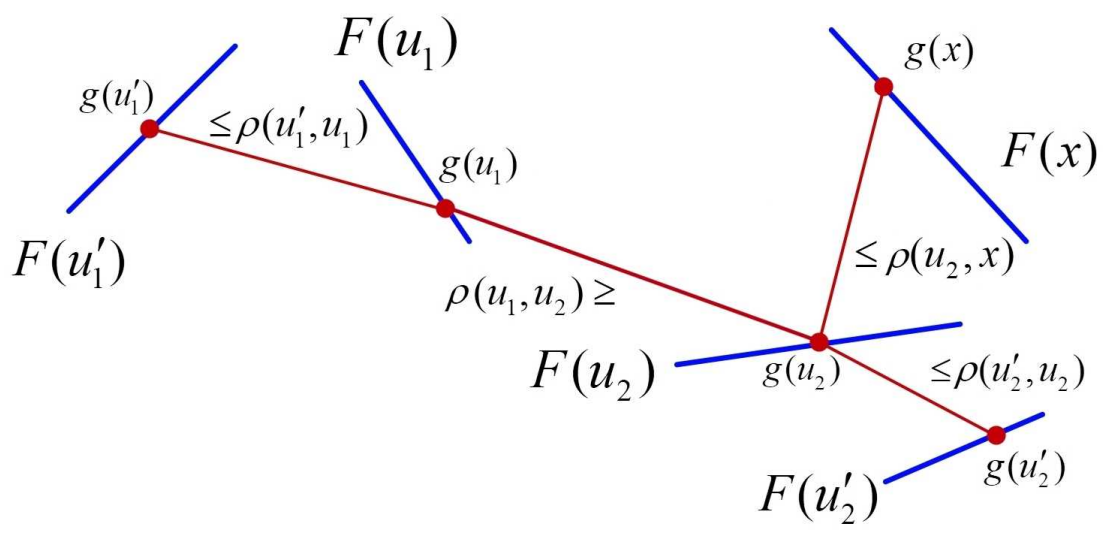

Fig. 26: The existence of a mapping $g$ which agrees with this diagram implies (4.18).

Indeed, let $\widetilde{\mathcal{M}}=\left\{u_{1}^{\prime}, u_{1}, x, u_{2}, u_{2}^{\prime}\right\}$. This property and definitions $4.16,4.17$ imply the existence of a mapping $g: \widetilde{\mathcal{M}} \rightarrow X$ with the following properties: $g(v) \in F(v)$ on $\widetilde{\mathcal{M}}$,

$$
\left\|g\left(u_{1}\right)-g\left(u_{1}^{\prime}\right)\right\| \leq \rho\left(u_{1}, u_{1}^{\prime}\right), \quad\left\|g\left(u_{1}\right)-g\left(u_{2}\right)\right\| \leq \rho\left(u_{1}, u_{2}\right),
$$

and

$$
\left\|g\left(u_{2}\right)-g\left(u_{2}^{\prime}\right)\right\| \leq \rho\left(u_{2}, u_{2}^{\prime}\right), \quad\left\|g\left(u_{2}\right)-g(x)\right\| \leq \rho\left(u_{2}, x\right) .
$$

We establish 4.14) by showing that

$$
g(x) \in F(x) \cap \widetilde{T}_{x}\left(u_{1}, u_{1}^{\prime}\right) \bigcap \widetilde{T}_{x}\left(u_{2}, u_{2}^{\prime}\right)
$$


In fact, from the above properties of $g$ it follows that $g(x) \in F(x)$. We also know that $g\left(u_{2}\right) \in F\left(u_{2}\right), g\left(u_{2}^{\prime}\right) \in F\left(u_{2}^{\prime}\right)$. Thanks to (4.19), (4.20) and 4.8),

$$
\left\|g\left(u_{2}\right)-g\left(u_{2}^{\prime}\right)\right\| \leq \rho\left(u_{2}, u_{2}^{\prime}\right) \leq \mathrm{d}\left(u_{2}, u_{2}^{\prime}\right) \quad \text { and } \quad\left\|g\left(u_{2}\right)-g(x)\right\| \leq \rho\left(u_{2}, x\right) \leq L \mathrm{~d}\left(u_{2}, x\right) .
$$

From these properties of $g$ and definition 4.15), we have

$$
g(x) \in\left[\left(F\left(u_{2}^{\prime}\right)+\mathrm{d}\left(u_{2}^{\prime}, u_{2}\right) B_{X}\right) \cap F\left(u_{2}\right)\right]+L \mathrm{~d}\left(u_{2}, x\right) B_{X}=\widetilde{T}_{x}\left(u_{2}, u_{2}^{\prime}\right) .
$$

It remains to show that $g(x) \in \widetilde{T}_{x}\left(u_{1}, u_{1}^{\prime}\right)$. As we know,

$$
g(x) \in F(x), \quad g\left(u_{1}\right) \in F\left(u_{1}\right), \quad \text { and } \quad g\left(u_{1}^{\prime}\right) \in F\left(u_{1}^{\prime}\right) .
$$

Furthermore, thanks to 4.19) and (4.8),

$$
\left\|g\left(u_{1}\right)-g\left(u_{1}^{\prime}\right)\right\| \leq \rho\left(u_{1}, u_{1}^{\prime}\right) \leq \mathrm{d}\left(u_{1}, u_{1}^{\prime}\right) .
$$

Let us estimate $\left\|g\left(u_{1}\right)-g(x)\right\|$. From 4.19, 4.20) and the triangle inequality, we have

$$
\begin{aligned}
\left\|g\left(u_{1}\right)-g(x)\right\| & \leq\left\|g\left(u_{1}\right)-g\left(u_{2}\right)\right\|+\left\|g\left(u_{2}\right)-g(x)\right\| \leq \rho\left(u_{1}, u_{2}\right)+\rho\left(u_{2}, x\right) \\
& \leq \rho\left(u_{1}, x\right)+\rho\left(x, u_{2}\right)+\rho\left(u_{2}, x\right)=\rho\left(u_{1}, x\right)+2 \rho\left(x, u_{2}\right) .
\end{aligned}
$$

Recall that $\rho\left(u_{1}, x\right) \geq \rho\left(u_{2}, x\right)$. This and 4.8 yield

$$
\left\|g\left(u_{1}\right)-g(x)\right\| \leq 3 \rho\left(u_{1}, x\right) \leq L \mathrm{~d}\left(u_{1}, x\right) .
$$

This inequality, 4.22, 4.23 and 4.15 imply the required property $g(x) \in \widetilde{T}_{x}\left(u_{1}, u_{1}^{\prime}\right)$ proving 4.21.

Thus, to complete the proof of the proposition, we have to prove that the sets $G_{i}$, $i=1, \ldots, 4$, have a common point.

See Fig. 27.

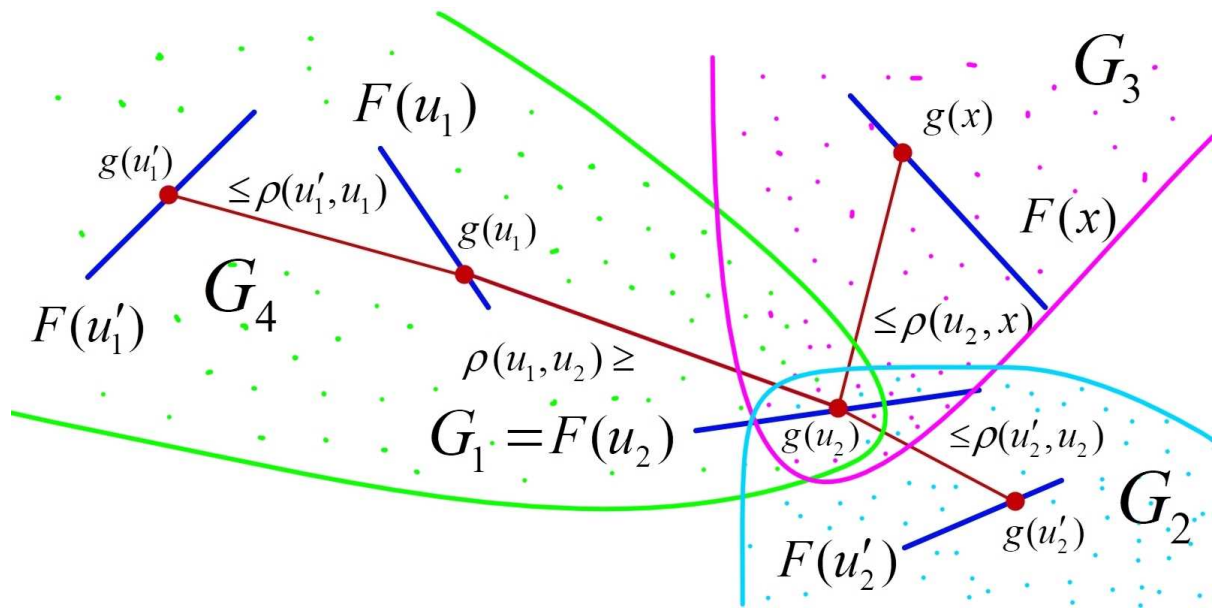

Fig. 27: The sets $G_{1}, G_{2}, G_{3}$ and $G_{4}$.

Note that $G_{1}=F\left(u_{2}\right) \in \mathcal{K}_{1}(X)$ so that, by Theorem 4.1, this property holds provided

$$
G_{1} \cap G_{i} \cap G_{j} \neq \emptyset \quad \text { for every } \quad 2 \leq i, j \leq 4, i \neq j .
$$


First prove that $G_{1} \cap G_{2} \cap G_{3} \neq \emptyset$.

Let $\mathcal{M}_{1}=\left\{u_{2}^{\prime}, u_{2}, x\right\}$. Thanks to Claim 4.3 , there exists a mapping $f_{1}: \mathcal{M}_{1} \rightarrow X$ with the following properties: $f_{1}(x) \in F(x), f_{1}\left(u_{2}\right) \in F\left(u_{2}\right), f_{1}\left(u_{2}^{\prime}\right) \in F\left(u_{2}^{\prime}\right)$,

$$
\left\|f_{1}\left(u_{2}\right)-f_{1}(x)\right\| \leq \rho\left(u_{2}, x\right) \text { and }\left\|f_{1}\left(u_{2}\right)-f_{1}\left(u_{2}^{\prime}\right)\right\| \leq \rho\left(u_{2}, u_{2}^{\prime}\right) .
$$

These properties of $f_{1}$ and definition 4.16 tell us that $f_{1}\left(u_{2}\right) \in G_{1} \cap G_{2} \cap G_{3}$ proving that the sets $G_{1}, G_{2}$ and $G_{3}$ have a common point.

Prove that $G_{1} \cap G_{2} \cap G_{4} \neq \emptyset$.

Let $\mathcal{M}_{2}=\left\{u_{1}^{\prime}, u_{1}, u_{2}^{\prime}, u_{2}\right\}$. Using Claim 4.3, we produce a mapping $f_{2}: \mathcal{M}_{2} \rightarrow X$ such that $f_{2}\left(u_{i}\right) \in F\left(u_{i}\right), f_{2}\left(u_{i}^{\prime}\right) \in F\left(u_{i}^{\prime}\right)$ for every $i=1,2,\left\|f_{2}\left(u_{1}\right)-f_{2}\left(u_{1}^{\prime}\right)\right\| \leq \rho\left(u_{1}, u_{1}^{\prime}\right)$,

$$
\left\|f_{2}\left(u_{1}\right)-f_{2}\left(u_{2}\right)\right\| \leq \rho\left(u_{1}, u_{2}\right) \quad \text { and } \quad\left\|f_{2}\left(u_{2}\right)-f_{2}\left(u_{2}^{\prime}\right)\right\| \leq \rho\left(u_{2}, u_{2}^{\prime}\right) .
$$

These properties of $f_{2}$ and (4.16, (4.17) yield $f_{2}\left(u_{2}\right) \in G_{1} \cap G_{2} \cap G_{4}$ proving that the sets $G_{1}, G_{2}$ and $G_{4}$ have a common point.

In the same way we show that $G_{1} \cap G_{3} \cap G_{4} \neq \emptyset$. (We set $\mathcal{M}_{3}=\left\{u_{1}^{\prime}, u_{1}, x, u_{2}\right\}$, produce a corresponding function $f_{3}: \mathcal{M}_{3} \rightarrow X$ and show that $f_{3}\left(u_{2}\right) \in G_{1} \cap G_{3} \cap G_{4}$.)

Thus, (4.24) holds, proving that the sets $G_{i}$ have a common point.

The proof of the proposition is complete.

In this section we set $\gamma_{0}=\gamma_{0}(L)=L \theta(L)$.

Proposition 4.8. For every $x, y \in \mathcal{M}$ the following inequality

$$
\mathrm{d}_{\mathrm{H}}\left(F^{[2]}(x), F^{[2]}(y)\right) \leq \gamma_{0}(L) \mathrm{d}(x, y)
$$

holds.

Proof. Let $x, y \in \mathcal{M}$. Thanks to 4.13,

$$
F^{[2]}(x)=\bigcap_{u, u^{\prime} \in \mathcal{M}} \widetilde{T}_{x}\left(u, u^{\prime}\right) \text { and } F^{[2]}(y)=\bigcap_{u, u^{\prime} \in \mathcal{M}} \widetilde{T}_{y}\left(u, u^{\prime}\right) .
$$

Recall that

$$
\widetilde{T}_{x}\left(u, u^{\prime}\right)=\left[\left(F\left(u^{\prime}\right)+\mathrm{d}\left(u^{\prime}, u\right) B_{X}\right) \cap F(u)\right]+L \mathrm{~d}(u, x) B_{X} .
$$

Recall that the set $\widetilde{T}_{x}\left(u, u^{\prime}\right)$ is the orbit of $x$ with respect to the diagram of Fig. 24.

We know that $F^{[2]}(x) \neq \emptyset$, see Proposition 4.7, and $\widetilde{T}_{x}(x, x)=F(x) \in \mathcal{K}_{1}(X)$. These properties, 4.25) and Lemma 4.4 tell us that

$$
F^{[2]}(x)+\gamma_{0}(L) \mathrm{d}(x, y) B_{X}=\bigcap_{u, u^{\prime} \in \mathcal{M}}\left\{\left[\widetilde{T}_{x}\left(u, u^{\prime}\right) \cap F(x)\right]+\gamma_{0}(L) \mathrm{d}(x, y) B_{X}\right\} .
$$

We fix $u, u^{\prime} \in \mathcal{M}$ and introduce a set

$$
\widetilde{A}=\left[\widetilde{T}_{x}\left(u, u^{\prime}\right) \bigcap F(x)\right]+\gamma_{0}(L) \mathrm{d}(x, y) B_{X} .
$$

Clearly, this set is the orbit of $y$ with respect to the diagram in Fig. 28. 


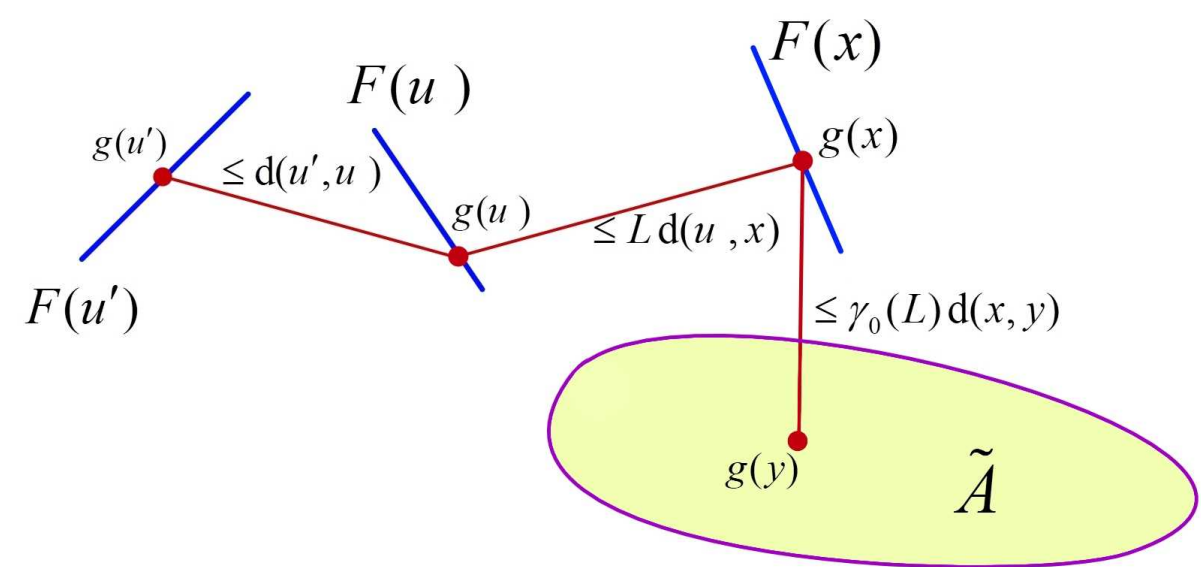

Fig. 28: $\widetilde{A}=\{g(y)\}$ where $g$ runs over all mappings which agree with this diagram.

We also introduce sets

$$
C_{1}=F(u), \quad C_{2}=F\left(u^{\prime}\right)+\mathrm{d}\left(u^{\prime}, u\right) B_{X}, \quad \text { and } \quad C=F(x) .
$$

Let

$$
\varepsilon=L \mathrm{~d}(x, y) \text { and } r=\mathrm{d}(x, u) \text {. }
$$

In these settings, $\gamma_{0}(L) \mathrm{d}(x, y)=\theta(L) \varepsilon$ and

$$
\widetilde{A}=\left[\widetilde{T}_{x}\left(u, u^{\prime}\right) \cap F(x)\right]+\gamma_{0}(L) \mathrm{d}(x, y) B_{X}=\left[\left\{\left(C_{1} \cap C_{2}\right)+L r B_{X}\right\} \cap C\right]+\theta(L) \varepsilon B_{X} .
$$

Our aim is to prove that $\widetilde{A} \supset F^{[2]}(y)=\bigcap\left\{\widetilde{T}_{y}\left(z, z^{\prime}\right): z, z^{\prime} \in \mathcal{M}\right\}$. See Fig. 29 .

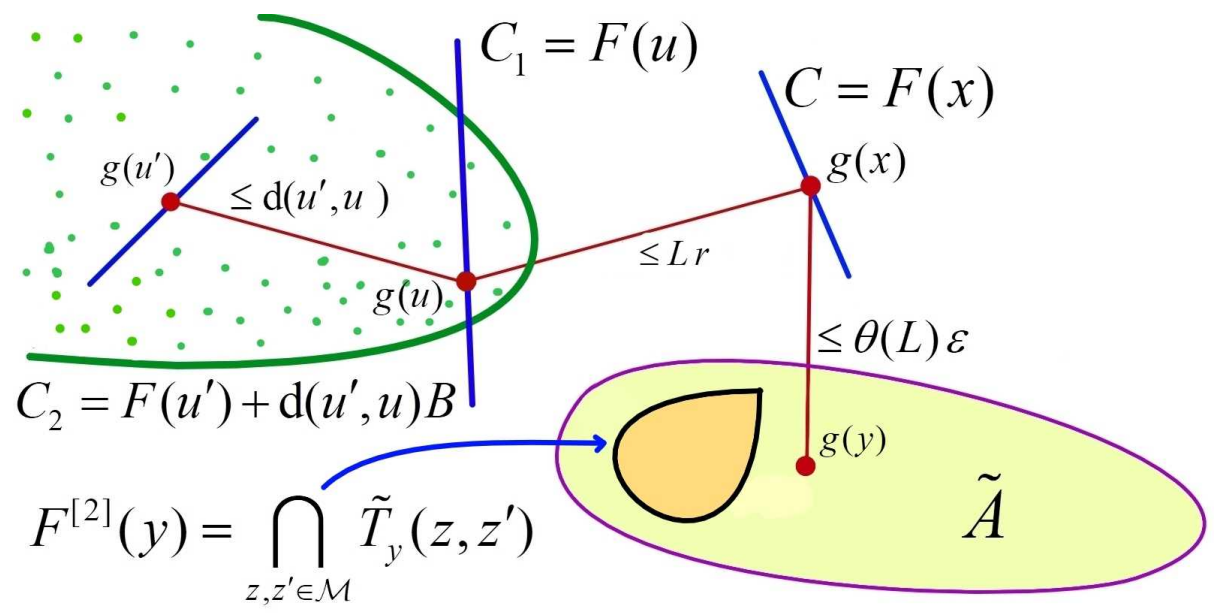

Fig. 29: The sets $C_{1}, C_{2}$ and $C$.

In fact, we prove that

$$
\widetilde{A} \supset \widetilde{T}_{y}\left(u, u^{\prime}\right) \cap \widetilde{T}_{y}\left(x, u^{\prime}\right) .
$$


Let us apply Proposition 4.2 to the set $\widetilde{A}$. To do this, we have to verify condition 4.1 , i.e., to show that

$$
C_{1} \cap C_{2} \cap\left(C+r B_{X}\right) \neq \emptyset \text {. }
$$

Let $\widetilde{\mathcal{M}}=\left\{x, u, u^{\prime}\right\}$. Thanks to Claim 4.3 , there exists a $\rho$-Lipschitz selection $f_{\widetilde{\mathcal{M}}}$ of the restriction $\left.F\right|_{\widetilde{\mathcal{M}}}$ with $\left\|f_{\widetilde{\mathcal{M}}}\right\|_{\operatorname{Lip}((\widetilde{\mathcal{M}} ; \rho), X)} \leq 1$. Thus, $f_{\widetilde{\mathcal{M}}}\left(u^{\prime}\right) \in F\left(u^{\prime}\right), f_{\widetilde{\mathcal{M}}}(u) \in F(u)$, $f_{\widetilde{\mathcal{M}}}(x) \in F(x)$,

$$
\left\|f_{\widetilde{\mathcal{M}}}\left(u^{\prime}\right)-f_{\widetilde{\mathcal{M}}}(u)\right\| \leq \rho\left(u^{\prime}, u\right) \text { and }\left\|f_{\widetilde{\mathcal{M}}}(x)-f_{\widetilde{\mathcal{M}}}(u)\right\| \leq \rho(x, u) .
$$

Let us prove that

$$
f_{\widetilde{\mathcal{M}}}(u) \in C_{1} \cap C_{2} \cap\left(C+r B_{X}\right) .
$$

Indeed, $f_{\widetilde{\mathcal{M}}}(u) \in F(u)=C_{1}$, see $(4.28)$. Furthermore, $f_{\widetilde{\mathcal{M}}}\left(u^{\prime}\right) \in F\left(u^{\prime}\right)$ and, thanks to (4.8), $\rho \leq \mathrm{d}$ on $\mathcal{M}$. Hence,

$$
\left\|f_{\widetilde{\mathcal{M}}}\left(u^{\prime}\right)-f_{\widetilde{\mathcal{M}}}(u)\right\| \leq \rho\left(u^{\prime}, u\right) \leq \mathrm{d}\left(u^{\prime}, u\right)
$$

proving that $f_{\widetilde{\mathcal{M}}}(u) \in C_{2}$, see 4.28 ). Finally, by 4.28 ) and 4.29$), f_{\widetilde{\mathcal{M}}}(x) \in F(x)=C$ and

$$
\left\|f_{\widetilde{\mathcal{M}}}(x)-f_{\widetilde{\mathcal{M}}}(u)\right\| \leq \rho(x, u) \leq \mathrm{d}(x, u)=r, \quad \text { so that } \quad f_{\widetilde{\mathcal{M}}}(u) \in C+r B_{X} .
$$

Thus, 4.31) is true so that property (4.30) holds. Furthermore, $C_{1}=F(u) \in \mathcal{K}_{1}(X)$, so that all conditions of the hypothesis of Proposition 4.2 are satisfied. By this proposition,

$$
\begin{aligned}
\widetilde{A} & =\left[\left\{\left(C_{1} \cap C_{2}\right)+L r B_{X}\right\} \cap C\right]+\theta(L) \varepsilon B_{X} \\
& \supset\left[\left(C_{1} \cap C_{2}\right)+(L r+\varepsilon) B_{X}\right] \cap\left[\left\{\left(C_{1}+r B_{X}\right) \cap C\right\}+\varepsilon B_{X}\right] \\
& =\widetilde{A_{1}} \cap \widetilde{A_{2}} .
\end{aligned}
$$

Prove that $\widetilde{A_{i}} \supset F^{[2]}(y)$ for every $i=1,2$.

We begin with the set

$$
\widetilde{A_{1}}=C_{1} \cap C_{2}+(L r+\varepsilon) B_{X} .
$$

Thanks to 4.28 and 4.29,

$$
\widetilde{A_{1}}=\left[\left\{F\left(u^{\prime}\right)+\mathrm{d}\left(u^{\prime}, u\right) B_{X}\right\} \cap F(u)\right]+(L \mathrm{~d}(u, x)+L \mathrm{~d}(x, y)) B_{X} .
$$

By the triangle inequality,

$$
\mathrm{d}(u, x)+\mathrm{d}(x, y) \geq \mathrm{d}(u, y)
$$

so that

$$
\widetilde{A_{1}} \supset\left[\left\{F\left(u^{\prime}\right)+\mathrm{d}\left(u^{\prime}, u\right) B_{X}\right\} \cap F(u)\right]+L \mathrm{~d}(u, y) B_{X}=\widetilde{T}_{y}\left(u, u^{\prime}\right), \quad \text { see 4.26). }
$$

But, by 4.25$), \widetilde{T}_{y}\left(u, u^{\prime}\right) \supset F^{[2]}(y)$ which implies the required inclusion $\widetilde{A}_{1} \supset F^{[2]}(y)$. See Fig. 30. 


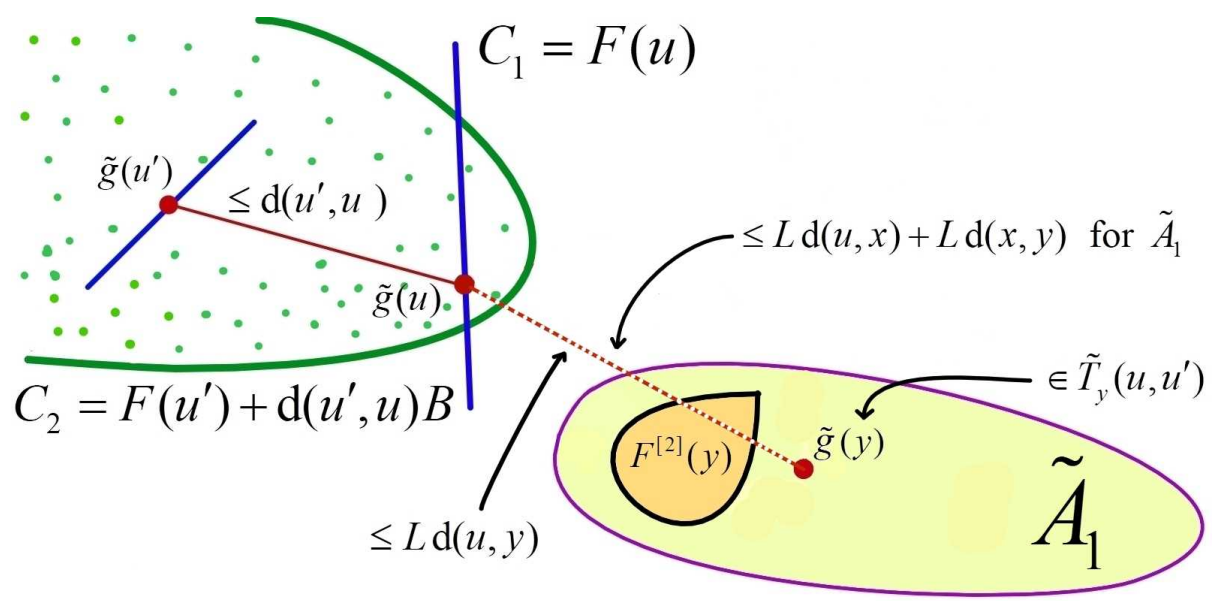

Fig. 30: The set $\widetilde{A_{1}}=C_{1} \cap C_{2}+(L r+\varepsilon) B_{X}$ containing the set $\widetilde{T}_{y}\left(u, u^{\prime}\right)$.

We turn to the set $\widetilde{A_{2}}=\left[\left(C_{1}+r B_{X}\right) \cap C\right]+\varepsilon B_{X}$. By 4.12$\left.), 4.28\right)$ and 4.29$)$,

$$
\widetilde{A_{2}}=\left[\left(F(u)+\mathrm{d}(u, x) B_{X}\right) \cap F(x)\right]+L \mathrm{~d}(x, y) B_{X}=T_{y}(x, u) .
$$

Thanks to 4.25$), \widetilde{T}_{y}(u, x) \supset F^{[2]}(y)$ proving that $\widetilde{A}_{2} \supset F^{[2]}(y)$.

Thus,

$$
\widetilde{A}=\left[\widetilde{T}_{x}\left(u, u^{\prime}\right) \cap F(x)\right]+\gamma_{0}(L) \mathrm{d}(x, y) B_{X} \supset \widetilde{A_{1}} \cap \widetilde{A_{2}} \supset F^{[2]}(y) \quad \text { for every } \quad u, u^{\prime} \in \mathcal{M} .
$$

From this and representation 4.27 , we have $F^{[2]}(x)+\gamma_{0}(L) \mathrm{d}(x, y) B_{X} \supset F^{[2]}(y)$.

By interchanging the roles of $x$ and $y$ we obtain also

$$
F^{[2]}(y)+\gamma_{0}(L) \mathrm{d}(x, y) B_{X} \supset F^{[2]}(x) .
$$

These two inclusions and (1.2) imply the statement of the proposition.

We complete the proof of Theorem 1.10 as follows. We fix $\lambda_{1}, \lambda_{2}$ and $\gamma$ satisfying inequalities 1.10. Then, by Proposition 4.7, the set $F^{[2]}(x) \neq \emptyset$ for every $x \in \mathcal{M}$.

In turn, Proposition 4.8 tells us that $\mathrm{d}_{\mathrm{H}}\left(F^{[2]}(x), F^{[2]}(y)\right) \leq \gamma_{0}(L) \mathrm{d}(x, y)$ on $\mathcal{M}$. We recall that $L=\lambda_{2} / \lambda_{1}, \mathrm{~d}=\lambda_{1} \rho, \gamma_{0}(L)=L \theta(L)$ and $\theta(L)=(3 L+1) /(L-1)$. Hence,

$$
\mathrm{d}_{\mathrm{H}}\left(F^{[2]}(x), F^{[2]}(y)\right) \leq \gamma_{0}(L) \mathrm{d}(x, y)=L\left(\frac{3 L+1}{L-1}\right) \mathrm{d}(x, y)=\frac{\lambda_{2}\left(3 \lambda_{2}+\lambda_{1}\right)}{\left(\lambda_{2}-\lambda_{1}\right)} \rho(x, y) .
$$

This inequality and (1.10) imply (1.8).

Thus, (1.7) and (1.8) hold provided $\lambda_{1}, \lambda_{2}$ and $\gamma$ satisfy inequalities (1.10). In particular, we can set $\lambda_{1}=1, \lambda_{2}=3$ and $\gamma=\lambda_{2}\left(3 \lambda_{2}+\lambda_{1}\right) /\left(\lambda_{2}-\lambda_{1}\right)=15$.

Let now $X$ be a Euclidean space, and let $\lambda_{1}, \lambda_{2}$ and $\gamma$ be the parameters satisfying inequalities (1.11).

In this case, we replace in the above calculations the constant $\theta(L)=(3 L+1) /(L-1)$ with $\theta(L)=1+2 L / \sqrt{L^{2}-1}$. This leads us to the required estimate

$$
\mathrm{d}_{\mathrm{H}}\left(F^{[2]}(x), F^{[2]}(y)\right) \leq\left\{\lambda_{2}+2 \lambda_{2}^{2} /\left(\lambda_{2}^{2}-\lambda_{1}^{2}\right)^{\frac{1}{2}}\right\} \rho(x, y) \leq \gamma \rho(x, y)
$$

proving that (1.7) and (1.8) hold for $\lambda_{1}, \lambda_{2}$ and $\gamma$ satisfying (1.11).

The proof of Theorem 1.10 is complete. 


\section{The main theorem in $\ell_{\infty}^{2}$.}

5.1 The case $X=\mathbf{R}$.

Proposition 5.1. Let $(\mathcal{M}, \rho)$ be a pseudometric space. Let $m=1$ and let $X=\mathbf{R}$; thus, $\ell=\ell(m, X)=1$, see (1.1). In this case Conjecture 1.8 holds for every $\lambda_{1} \geq 1$ and $\gamma \geq 1$.

Thus, the following statement is true: Let $F$ be a set-valued mapping from $\mathcal{M}$ into the family $\mathcal{K}(\mathbf{R})$ of all closed bounded intervals in $\mathbf{R}$. Suppose that for every $x, y \in \mathcal{M}$ there exist points $g(x) \in F(x)$ and $g(y) \in F(y)$ such that $|g(x)-g(y)| \leq \rho(x, y)$.

Let $F^{[1]}(x), x \in \mathcal{M}$, be the $\lambda_{1}$-balanced refinement of the mapping $F$, i.e., the set

$$
F^{[1]}(x)=\bigcap_{z \in \mathcal{M}}\left[F(z)+\lambda_{1} \rho(x, z) I_{0}\right] \quad \text { where } I_{0}=[-1,1] .
$$

Then $F^{[1]}(x) \neq \emptyset$ for every $x \in \mathcal{M}$, and

$$
\mathrm{d}_{\mathrm{H}}\left(F^{[1]}(x), F^{[1]}(y)\right) \leq \gamma \rho(x, y) \quad \text { for all } \quad x, y \in \mathcal{M} .
$$

Note that Proposition 5.1 easily follows from the one dimensional Helly Theorem and a formula for a neighborhood of the intersection of intervals in $\mathbf{R}$. We formulate these statements in the following

Lemma 5.2. Let $\mathcal{K} \subset \mathcal{K}(\mathbf{R})$ be a collection of closed bounded intervals in $\mathbf{R}$.

(a) (Helly's Theorem in $\mathbf{R}$.) If the intersection of every two intervals from $\mathcal{K}$ is nonempty, then there exists a point in $\mathbf{R}$ common to all of the family $\mathcal{K}$.

(b) Suppose that $\cap\{K: K \in \mathcal{K}\} \neq \emptyset$. Then for every $r \geq 0$ we have

$$
\left(\bigcap_{K \in \mathcal{K}} K\right)+r I_{0}=\bigcap_{K \in \mathcal{K}}\left\{K+r I_{0}\right\} .
$$

Proof of part (b). In Lemma 3.4 we proved an analog of property $(b)$ for $\mathbf{R}^{2}$. We prove part $(b)$ by replacing in that proof the Helly Theorem 2.5 with the one dimensional Helly Theorem formulated in part $(a)$ of the present lemma. We leave the details to the interested reader.

Proof of Proposition 5.1. We have to prove that the set $F^{[1]}(x)$ is non-empty for each $x \in \mathcal{M}$, and for every $x, y \in \mathcal{M}$

$$
\mathrm{d}_{\mathrm{H}}\left(F^{[1]}(x), F^{[1]}(y)\right) \leq \gamma \rho(x, y) .
$$

We know that the restriction $\left.F\right|_{\mathcal{M}^{\prime}}$ of $F$ to every two point subset $\mathcal{M}^{\prime} \subset \mathcal{M}$ has a Lipschitz selection $f_{\mathcal{M}^{\prime}}: \mathcal{M}^{\prime} \rightarrow \mathbf{R}$ with $\left\|f_{\mathcal{M}^{\prime}}\right\|_{\operatorname{Lip}\left(\mathcal{M}^{\prime}, \mathbf{R}\right)} \leq \gamma$. Thus, for every $z, z^{\prime} \in \mathcal{M}$ there exist points

$$
g(z) \in F(z), g\left(z^{\prime}\right) \in F\left(z^{\prime}\right) \text { such that }\left|g(z)-g\left(z^{\prime}\right)\right| \leq \gamma \rho\left(z, z^{\prime}\right)
$$

We recall that the set-valued mapping $F^{[1]}$ is defined by formula $(5.1)$. 
Prove that $F^{[1]}(x) \neq \emptyset$ for every $x \in \mathcal{M}$. Indeed, thanks to $(5.1)$ and Helly's Theorem for intervals (part (i) of Lemma 5.2), $F^{[1]}(x) \neq \emptyset$ provided

$$
\left(F(z)+\gamma \rho(x, z) I_{0}\right) \cap\left(F\left(z^{\prime}\right)+\gamma \rho\left(x, z^{\prime}\right) I_{0}\right) \neq \emptyset
$$

for every $z, z^{\prime} \in \mathcal{M}$.

We know that there exist points $g(z)$ and $g\left(z^{\prime}\right)$ satisfying (5.3). Let

$$
a=\min \left\{g(z)+\gamma \rho(z, x), g\left(z^{\prime}\right)+\gamma \rho\left(z^{\prime}, x\right)\right\} .
$$

Thanks to the inequality $\left|g(z)-g\left(z^{\prime}\right)\right| \leq \gamma \rho\left(z, z^{\prime}\right)$, we have

$$
g(z)=\min \left\{g(z), g\left(z^{\prime}\right)+\gamma \rho\left(z^{\prime}, z\right)\right\}
$$

so that, by the triangle inequality,

$$
|a-g(z)| \leq \max \left\{\gamma \rho(z, x),\left|\gamma \rho\left(z^{\prime}, x\right)-\gamma \rho\left(z^{\prime}, z\right)\right|\right\}=\gamma \rho(z, x) .
$$

We also know that $g(z) \in F(z)$, see (5.3), so that $a \in F(z)+\gamma \rho(x, z) I_{0}$.

In the same way we show that $a \in F\left(z^{\prime}\right)+\gamma \rho\left(x, z^{\prime}\right) I_{0}$ proving property (5.4).

Prove that

$$
F^{[1]}(x)+\gamma \rho(x, y) I_{0} \supset F^{[1]}(y) \quad \text { for every } \quad x, y \in \mathcal{M} .
$$

We know that $F^{[1]}(x) \neq \emptyset$ which enables us to apply part (b) of Lemma 5.2 to the left hand side of (5.5). This lemma and definition (5.1) tell us that

$$
\begin{aligned}
F^{[1]}(x)+\gamma \rho(x, y) I_{0} & =\left\{\bigcap_{z \in \mathcal{M}}\left[F(z)+\gamma \rho(x, z) I_{0}\right]\right\}+\gamma \rho(x, y) I_{0} \\
& =\bigcap_{z \in \mathcal{M}}\left[F(z)+(\gamma \rho(x, z)+\gamma \rho(x, y)) I_{0}\right]
\end{aligned}
$$

so that, thanks to the triangle inequality,

$$
F^{[1]}(x)+\gamma \rho(x, y) I_{0} \supset \bigcap_{z \in \mathcal{M}}\left[F(z)+\gamma \rho(y, z) I_{0}\right]=F^{[1]}(y)
$$

proving (5.5). By interchanging the roles of $x$ and $y$ we obtain also

$$
F^{[1]}(y)+\gamma \rho(x, y) I_{0} \supset F^{[1]}(x) .
$$

These two inclusions prove the required inequality (5.2).

The proof of Proposition 5.1 is complete.

\subsection{Rectangular hulls of plane convex sets.}

Let us fix some additional notation. We let $\mathfrak{R}\left(\mathbf{R}^{2}\right)$ denote the family of all bounded closed rectangles in $\mathbf{R}^{2}$ with sides parallel to the coordinate axes $O x_{1}$ and $O x_{2}$.

Let $Q_{0}=B_{X}$ be the unit ball of the Banach space $X=\ell_{\infty}^{2}$, i.e., the square $Q_{0}=[-1,1]^{2}$. Given $a \in \mathbf{R}^{2}$ and $r \geq 0$, we set $r Q_{0}=[-r, r]^{2}$ and $Q(a, r)=r Q_{0}+a$. 
Definition 5.3. Let $S$ be a non-empty bounded convex closed subset in $\mathbf{R}^{2}$. We set

$$
\mathcal{H}[S]=\bigcap\left\{\Pi: \Pi \in \mathfrak{R}\left(\mathbf{R}^{2}\right), \Pi \supset S\right\},
$$

and refer to $\mathcal{H}[S]$ as the "rectangular hull" of the set $S$.

Note the following useful representation of the rectangular hull which easily follows from Definition 5.3 .

$$
\mathcal{H}[S]=\left(S+O x_{1}\right) \bigcap\left(S+O x_{2}\right)
$$

In the next section we need the following auxiliary

Lemma 5.4. Let $K_{1}, K_{2} \in \mathcal{K}\left(\mathbf{R}^{2}\right)$ be two convex compacts in $\mathbf{R}^{2}$ with non-empty intersection. Let $\tau \geq 0$ and let $Q=[-\tau, \tau]^{2}$. Then

$$
\left(K_{1} \cap K_{2}\right)+Q=\left(K_{1}+Q\right) \bigcap\left(K_{2}+Q\right) \cap \mathcal{H}\left[\left(K_{1} \cap K_{2}\right)+Q\right]
$$

Proof. Obviously, the right hand side of (5.7) contains its left hand side.

Let us prove the converse statement. Fix a point

$$
a \in\left(K_{1}+Q\right) \cap\left(K_{2}+Q\right) \cap \mathcal{H}\left[K_{1} \cap K_{2}+Q\right]
$$

Our aim is to prove that $a \in\left(K_{1} \cap K_{2}\right)+Q$. Clearly, this property holds if and only if $Q(a, \tau) \cap K_{1} \cap K_{2} \neq \emptyset$. It is also clear that $Q(a, \tau)=\Pi_{1}(a) \cap \Pi_{2}(a)$ where

$$
\Pi_{i}(a)=Q(a, \tau)+O x_{i}, \quad i=1,2 .
$$

Thus, $a \in\left(K_{1} \cap K_{2}\right)+Q$ provided

$$
K_{1} \cap K_{2} \cap \Pi_{1}(a) \cap \Pi_{2}(a) \neq \emptyset .
$$

See Fig. 31.

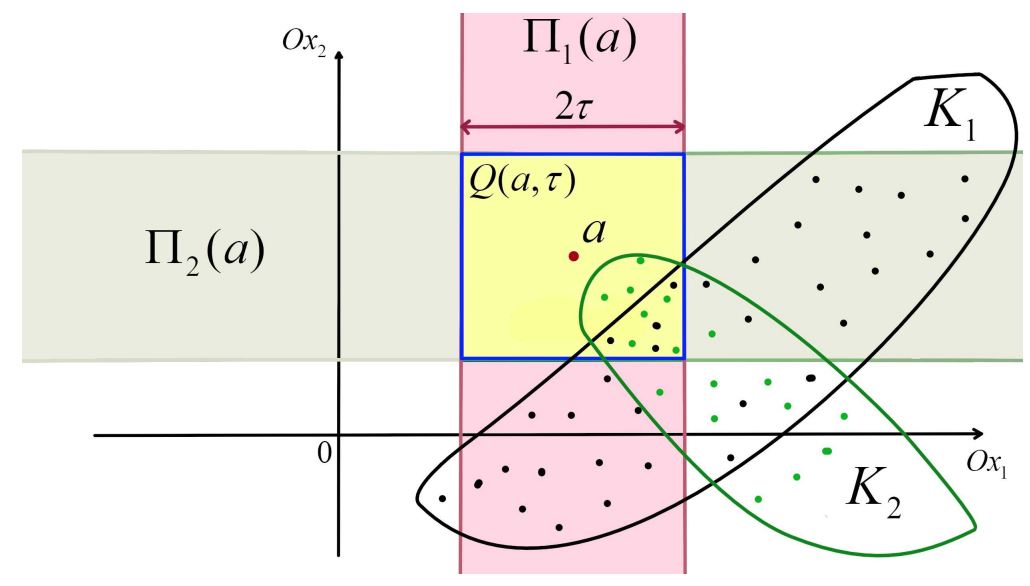

Fig. 31: TEXT 
Thanks to Theorem 2.5, the family of sets $\left\{K_{1}, K_{2}, \Pi_{1}(a), \Pi_{2}(a)\right\}$ has a common point provided any three members of this family have a non-empty intersection.

Prove that it is true for $a$ satisfying (5.8). Indeed, $a \in K_{i}+Q$, so that $K_{i} \cap Q(a, \tau) \neq \emptyset$, $i=1,2$. Hence,

$$
K_{i} \cap \Pi_{1}(a) \cap \Pi_{2}(a)=K_{i} \cap Q(a, \tau) \neq \emptyset, \quad i=1,2 .
$$

Next, thanks to (5.6) and (5.8), for every $i=1,2$,

$$
a \in \mathcal{H}\left[\left(K_{1} \cap K_{2}\right)+Q\right] \subset\left(K_{1} \cap K_{2}\right)+Q+O x_{i} .
$$

Hence, $K_{1} \cap K_{2} \cap \Pi_{i}(x) \neq \emptyset, i=1,2$, and the proof of the lemma is complete.

\subsection{Balanced refinements of set-valued mappings in $\ell_{\infty}^{2}$.}

In this section we refine the result of Theorem 1.9 for the space $X=\ell_{\infty}^{2}$.

Theorem 5.5. In the settings of Theorem 1.9 properties $(1.7)$ and $(1.8)$ hold provided $X=\ell_{\infty}^{2}$,

$$
\lambda_{1} \geq 1, \quad \lambda_{2} \geq 3 \lambda_{1}, \quad \text { and } \quad \gamma \geq \lambda_{2}\left(3 \lambda_{2}+\lambda_{1}\right) /\left(\lambda_{2}-\lambda_{1}\right)
$$

In particular, (1.7) and (1.8) hold whenever $\lambda_{1}=1, \lambda_{2}=3$ and $\gamma=15$.

Proof. We mainly follow the scheme of the proof of Theorem 1.9 given in Section 3.

Let $F: \mathcal{M} \rightarrow \mathcal{K}\left(\mathbf{R}^{2}\right)$ be a set-valued mapping satisfying the hypothesis of Theorem 5.5. Thus, the next statement is true.

Claim 5.6. For every $\mathcal{M}^{\prime} \subset \mathcal{M}, \# \mathcal{M}^{\prime} \leq 4$, the restriction $\left.F\right|_{\mathcal{M}^{\prime}}$ of $F$ to $\mathcal{M}^{\prime}$ has a $\rho$ Lipschitz selection $f_{\mathcal{M}^{\prime}}: \mathcal{M}^{\prime} \rightarrow \ell_{\infty}^{2}$ with $\rho$-Lipschitz seminorm $\left\|f_{\mathcal{M}^{\prime}}\right\|_{\operatorname{Lip}\left(\left(\mathcal{M}^{\prime}, \rho\right), \ell_{\infty}^{2}\right)} \leq 1$.

Let $\lambda_{1}$ and $\lambda_{2}$ be positive constants satisfying inequalities (5.9). We set $L=\lambda_{2} / \lambda_{1}$; thus, $L \geq 3$. Then we introduce a pseudometric on $\mathcal{M}$ defined by $\mathrm{d}(x, y)=\lambda_{1} \rho(x, y)$, $x, y \in \mathcal{M}$.

We let $F^{[1]}$ and $F^{[2]}$ denote the first and the second order $(\{1, L\}, \mathrm{d})$-balanced refinements of $F$ respectively. See Definition 1.6 . Thus,

$$
F^{[1]}(x)=\bigcap_{z \in \mathcal{M}}\left[F(z)+\mathrm{d}(x, z) Q_{0}\right] \quad \text { and } \quad F^{[2]}(x)=\bigcap_{z \in \mathcal{M}}\left[F^{[1]}(z)+L \mathrm{~d}(x, z) Q_{0}\right], \quad x \in \mathcal{M} .
$$

We also recall that $e\left(\mathfrak{M}, \ell_{\infty}^{2}\right)=1$. In this case, Lemma 3.5 and Proposition 3.8 tell us that $F^{[1]}(x) \neq \emptyset$ and $F^{[2]}(x) \neq \emptyset$ for every $x \in \mathcal{M}$.

Let

$$
\tilde{\gamma}(L)=L \theta(L) \text { where } \theta(L)=(3 L+1) /(L-1) .
$$

Prove that

$$
\mathrm{d}_{\mathrm{H}}\left(F^{[2]}(x), F^{[2]}(y)\right) \leq \tilde{\gamma}(L) \mathrm{d}(x, y) \text { for every } x, y \in \mathcal{M} .
$$


We recall that, thanks to formula 3.9$), F^{[2]}(x)=\bigcap\left\{T_{x}\left(u, u^{\prime}, u^{\prime \prime}\right): u, u^{\prime}, u^{\prime \prime} \in \mathcal{M}\right\}$ where, given $u, u^{\prime}, u^{\prime \prime} \in \mathcal{M}$,

$$
T_{x}\left(u, u^{\prime}, u^{\prime \prime}\right)=\left\{\left[F\left(u^{\prime}\right)+\mathrm{d}\left(u^{\prime}, u\right) Q_{0}\right] \cap\left[F\left(u^{\prime \prime}\right)+\mathrm{d}\left(u^{\prime \prime}, u\right) Q_{0}\right]\right\}+L \mathrm{~d}(u, x) Q_{0} .
$$

In particular, $T_{x}\left(u, u^{\prime}, u^{\prime \prime}\right) \neq \emptyset$ for all $u, u^{\prime}, u^{\prime \prime} \in \mathcal{M}$ (because $F^{[2]}(x) \neq \emptyset$ ).

The next lemma is a refinement of the formula 3.27 for the special case of $X=\ell_{\infty}^{2}$.

Lemma 5.7. Let $\tau>0$ and let $Q=\tau Q_{0}=[-\tau, \tau]^{2}$. Then, for every $x \in \mathcal{M}$, we have

$$
F^{[2]}(x)+Q=\bigcap_{v, u, u^{\prime}, u^{\prime \prime} \in \mathcal{M}}\left\{\left[T_{x}\left(u, u^{\prime}, u^{\prime \prime}\right) \cap\left(F(v)+\mathrm{d}(x, v) Q_{0}\right)\right]+Q\right\} .
$$

Proof. Lemma 3.9 tells us that

$$
F^{[2]}(x)+Q=\bigcap\left\{\left[T_{x}\left(u_{1}, u_{1}^{\prime}, u_{1}^{\prime \prime}\right) \bigcap T_{x}\left(u_{2}, u_{2}^{\prime}, u_{2}^{\prime \prime}\right)\right]+Q\right\}
$$

where the intersection is taken over all $u_{i}, u_{i}^{\prime}, u_{i}^{\prime \prime} \in \mathcal{M}, i=1,2$. Note also that, by (5.11),

$$
F(v)+\mathrm{d}(x, v) Q_{0}=T_{x}(x, v, v) .
$$

From this and $(5.13)$ it follows that the right hand side of $(5.12)$ contains its left hand side.

Prove the converse statement. Fix a point

$$
a \in \bigcap_{v, u, u^{\prime}, u^{\prime \prime} \in \mathcal{M}}\left\{\left[T_{x}\left(u, u^{\prime}, u^{\prime \prime}\right) \cap\left(F(v)+\mathrm{d}(x, v) Q_{0}\right)\right]+Q\right\}
$$

and show that $a \in F^{[2]}(x)+Q$. In view of formula 5.13 , it suffice to prove that for every $u_{1}, u_{1}^{\prime}, u_{1}^{\prime \prime}, u_{2}, u_{2}^{\prime}, u_{2}^{\prime \prime} \in \mathcal{M}$ the point a belongs to the set $A$ defined by

$$
A=\left[T_{x}\left(u_{1}, u_{1}^{\prime}, u_{1}^{\prime \prime}\right) \bigcap T_{x}\left(u_{2}, u_{2}^{\prime}, u_{2}^{\prime \prime}\right)\right]+Q .
$$

To see this, given $i \in\{1,2\}$ we introduce the following sets: $Q_{i}=L \mathrm{~d}\left(u_{i}, x\right) Q_{0}$,

$$
K_{i}^{\prime}=F\left(u_{i}^{\prime}\right)+\mathrm{d}\left(u_{i}, u_{i}^{\prime}\right) Q_{0} \quad \text { and } \quad K_{i}^{\prime \prime}=F\left(u_{i}^{\prime \prime}\right)+\mathrm{d}\left(u_{i}, u_{i}^{\prime \prime}\right) Q_{0} .
$$

In these settings, $T_{x}\left(u_{i}, u_{i}^{\prime}, u_{i}^{\prime \prime}\right)=K_{i}^{\prime} \cap K_{i}^{\prime \prime}+Q_{i}, i=1$, 2. See (5.11).

Note that $K_{i}^{\prime} \cap K_{i}^{\prime \prime} \neq \emptyset$ because $T_{x}\left(u_{i}, u_{i}^{\prime}, u_{i}^{\prime \prime}\right) \neq \emptyset$. Therefore, thanks to Lemma 5.4 ,

$$
T_{x}\left(u_{i}, u_{i}^{\prime}, u_{i}^{\prime \prime}\right)=\left(K_{i}^{\prime}+Q_{i}\right) \bigcap\left(K_{i}^{\prime \prime}+Q_{i}\right) \bigcap \mathcal{H}\left[T_{x}\left(u_{i}, u_{i}^{\prime}, u_{i}^{\prime \prime}\right)\right], \quad i=1,2 .
$$

Now, let us introduce the following families of sets:

$\mathcal{K}^{+}=\left\{K_{i}^{\prime}+Q_{i}, K_{i}^{\prime \prime}+Q_{i}: i=1,2\right\}, \mathcal{K}^{++}=\left\{\mathcal{H}\left[T_{x}\left(u_{i}, u_{i}^{\prime}, u_{i}^{\prime \prime}\right)\right]: i=1,2\right\}, \mathcal{K}=\mathcal{K}^{+} \cup \mathcal{K}^{++}$.

Then, thanks to (5.15) and 5.17), $A=[\cap\{K: K \in \mathcal{K}\}]+Q$.

We recall that, thanks to Proposition 3.8, the set $F^{[2]}(x) \neq \emptyset$ so that the left hand side of (5.13) is non-empty as well. From this and $(5.15)$ it follows that $A \neq \emptyset$ proving that $\cap\{K: K \in \mathcal{K}\} \neq \emptyset$. Therefore, thanks to Lemma 3.4 .

$$
A=\bigcap\left\{\left[K \cap K^{\prime}\right]+Q: K, K^{\prime} \in \mathcal{K}\right\} .
$$


Thus, to prove that $a \in A$ it suffice to show that $a \in K \cap K^{\prime}+Q$ for every $K, K^{\prime} \in \mathcal{K}$.

To do this, first let us note that, thanks to (5.16),

$$
K_{i}^{\prime}+Q_{i}=F\left(u_{i}^{\prime}\right)+\mathrm{d}\left(u_{i}, u_{i}^{\prime}\right) Q_{0}+L \mathrm{~d}\left(u_{i}, x\right) Q_{0} \supset F\left(u_{i}^{\prime}\right)+\left(\mathrm{d}\left(u_{i}, u_{i}^{\prime}\right)+\mathrm{d}\left(u_{i}, x\right)\right) Q_{0}
$$

for every $i=1,2$. Therefore, thanks to the triangle inequality,

$$
K_{i}^{\prime}+Q_{i} \supset F\left(u_{i}^{\prime}\right)+\mathrm{d}\left(u_{i}^{\prime}, x\right) Q_{0} .
$$

In the same way we prove that

$$
K_{i}^{\prime \prime}+Q_{i} \supset F\left(u_{i}^{\prime \prime}\right)+\mathrm{d}\left(u_{i}^{\prime \prime}, x\right) Q_{0}, \quad i=1,2 .
$$

Furthermore, we know that

$$
\mathcal{H}\left[T_{x}\left(u_{i}, u_{i}^{\prime}, u_{i}^{\prime \prime}\right)\right] \supset T_{x}\left(u_{i}, u_{i}^{\prime}, u_{i}^{\prime \prime}\right), \quad i=1,2 .
$$

On the other hand, property (5.14) tells us that

$$
\left.a \in T_{x}\left(u, u^{\prime}, u^{\prime \prime}\right) \bigcap\left[F(v)+\mathrm{d}(x, v) Q_{0}\right)\right]+Q \quad \text { for every } \quad u, u^{\prime}, u^{\prime \prime}, v \in \mathcal{M} .
$$

Combining this property with (5.18), (5.19) and (5.20), we conclude that

$$
a \in K \cap K^{\prime}+Q \text { whenever either } K \in \mathcal{K}^{+}, K^{\prime} \in \mathcal{K}^{++} \text {or } K, K^{\prime} \in \mathcal{K}^{+} \text {. }
$$

It remains to prove that

$$
a \in H_{1} \cap H_{2}+Q \text { where } H_{i}=\mathcal{H}\left[T_{x}\left(u_{i}, u_{i}^{\prime}, u_{i}^{\prime \prime}\right)\right], \quad i=1,2 .
$$

It is immediate from Lemma 5.2 , part (b), that

$$
H_{1} \cap H_{2}+Q=\left(H_{1}+Q\right) \cap\left(H_{2}+Q\right)
$$

so that

$$
H_{1} \cap H_{2}+Q=\left\{\mathcal{H}\left[T_{x}\left(u_{1}, u_{1}^{\prime}, u_{1}^{\prime \prime}\right)\right]+Q\right\} \cap\left\{\mathcal{H}\left[T_{x}\left(u_{2}, u_{2}^{\prime}, u_{2}^{\prime \prime}\right)\right]+Q\right\} .
$$

From this and (5.20), we have

$$
H_{1} \cap H_{2}+Q \supset\left\{T_{x}\left(u_{1}, u_{1}^{\prime}, u_{1}^{\prime \prime}\right)+Q\right\} \cap\left\{T_{x}\left(u_{2}, u_{2}^{\prime}, u_{2}^{\prime \prime}\right)+Q\right\} .
$$

But, thanks to (5.21), $a \in T_{x}\left(u_{i}, u_{i}^{\prime}, u_{i}^{\prime \prime}\right)+Q, i=1,2$. Combining this property with (5.23), we obtain the required property (5.22) completing the proof of the lemma.

We are in a position to prove inequality (5.10). Our proof will follow the scheme of the proof of Proposition 3.10 .

Let $x, y \in \mathcal{M}$, and let $\tau=\tilde{\gamma}(L) \mathrm{d}(x, y)$. (Recall that $\tilde{\gamma}(L)=L \theta(L)$ and $\mathrm{d}=\lambda_{1} \rho$.)

Lemma 5.7 tells us that

$$
F^{[2]}(x)+\tau Q_{0}=\bigcap_{v, u, u^{\prime}, u^{\prime \prime} \in \mathcal{M}}\left\{\left[T_{x}\left(u, u^{\prime}, u^{\prime \prime}\right) \cap\left(F(v)+\mathrm{d}(x, v) Q_{0}\right)\right]+\tau Q_{0}\right\} .
$$


Let us fix elements $u, u^{\prime}, u^{\prime \prime}, v \in \mathcal{M}$ and a set

$$
\widetilde{A}=\left[T_{x}\left(u, u^{\prime}, u^{\prime \prime}\right) \bigcap\left(F(v)+\mathrm{d}(x, v) Q_{0}\right)\right]+\tau Q_{0} .
$$

Our goal is to show that

$$
\widetilde{A} \supset F^{[2]}(y)=\bigcap_{z, z^{\prime}, z^{\prime \prime} \in \mathcal{M}} T_{y}\left(z, z^{\prime}, z^{\prime \prime}\right) . \quad \text { See (3.9). }
$$

In fact, we will prove a stronger imbedding:

$$
\widetilde{A} \supset T_{y}\left(u, u^{\prime}, u^{\prime \prime}\right) \bigcap T_{y}\left(x, u^{\prime}, v\right) \bigcap T_{y}\left(x, u^{\prime \prime}, v\right) .
$$

We also note that the set $\widetilde{A}$ is the orbit of the element $y$ with respect to the diagram shown in Fig. 32.

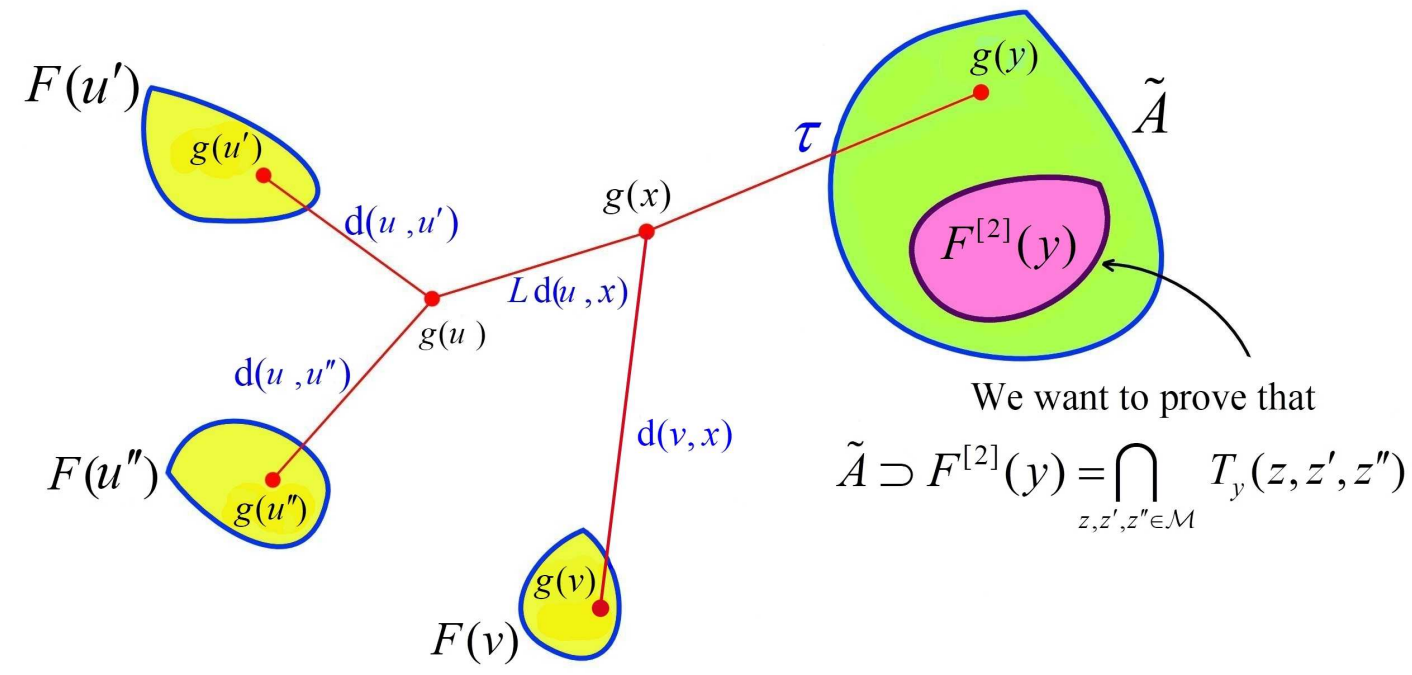

Fig. 32: $\widetilde{A}=\{g(y)\}$ where $g$ runs over all mappings which agree with this diagram.

Let

$$
C_{1}=F\left(u^{\prime}\right)+\mathrm{d}\left(u^{\prime}, u\right) Q_{0}, \quad C_{2}=F\left(u^{\prime \prime}\right)+\mathrm{d}\left(u^{\prime \prime}, u\right) Q_{0}, \quad C=F(v)+\mathrm{d}(x, v) Q_{0},
$$

and let $\varepsilon=L \mathrm{~d}(x, y)$ and $r=\mathrm{d}(u, x)$. Then

$$
\tau=\tilde{\gamma}(L) \mathrm{d}(x, y)=L \theta(L) \mathrm{d}(x, y)=\theta(L) \varepsilon .
$$

In these settings, $T_{x}\left(u, u^{\prime}, u^{\prime \prime}\right)=\left(C_{1} \cap C_{2}\right)+\operatorname{Lr} Q_{0}$, see (5.11), and

$$
\widetilde{A}=\left[\left\{\left(C_{1} \cap C_{2}\right)+\operatorname{Lr} Q_{0}\right\} \cap C\right]+\theta(L) \varepsilon Q_{0} . \quad \text { See (5.25). }
$$

Let us apply Proposition 2.6 to the sets $C_{1}, C_{2}$ and $C$ defined by (5.26). To do this, we have to verify condition (2.22) of that proposition, i.e., to prove that

$$
C_{1} \cap C_{2} \cap\left(C+r Q_{0}\right) \neq \emptyset .
$$


Let $\mathcal{M}^{\prime}=\left\{u, u^{\prime}, v\right\}$. Then, thanks to Claim 5.6, there exists a $\rho$-Lipschitz selection $f_{\mathcal{M}^{\prime}}: \mathcal{M}^{\prime} \rightarrow \ell_{\infty}^{2}$ of the restriction $\left.F\right|_{\mathcal{M}^{\prime}}$ with $\left\|f_{\mathcal{M}^{\prime}}\right\|_{\operatorname{Lip}\left(\left(\mathcal{M}^{\prime}, \rho\right), \ell_{\infty}^{2}\right)} \leq 1$.

Because $e\left(\mathfrak{M}, \ell_{\infty}^{2}\right)=1$ and $\mathrm{d}=\lambda_{1} \rho \geq \rho$, the mapping $f_{\mathcal{M}^{\prime}}: \mathcal{M}^{\prime} \rightarrow \ell_{\infty}^{2}$ can be extended to a d-Lipschitz mapping $\tilde{f}: \mathcal{M} \rightarrow \ell_{\infty}^{2}$ defined on all of $\mathcal{M}$ with d-Lipschitz seminorm

$$
\|\tilde{f}\|_{\operatorname{Lip}\left((\mathcal{M}, \mathrm{d}), \ell_{\infty}^{2}\right)} \leq\left\|f_{\mathcal{M}^{\prime}}\right\|_{\operatorname{Lip}\left(\left(\mathcal{M}^{\prime}, \rho\right), \ell_{\infty}^{2}\right)} \leq 1 .
$$

Thus, $\tilde{f}\left(u^{\prime}\right)=f_{\mathcal{M}^{\prime}}\left(u^{\prime}\right) \in F\left(u^{\prime}\right), \tilde{f}\left(u^{\prime \prime}\right)=f_{\mathcal{M}^{\prime}}\left(u^{\prime \prime}\right) \in F\left(u^{\prime \prime}\right), \tilde{f}(v)=f_{\mathcal{M}^{\prime}}(v) \in F(v)$,

$$
\left\|\tilde{f}\left(u^{\prime}\right)-\tilde{f}(u)\right\| \leq \mathrm{d}\left(u^{\prime}, u\right), \quad\left\|\tilde{f}\left(u^{\prime \prime}\right)-\tilde{f}(u)\right\| \leq \mathrm{d}\left(u^{\prime \prime}, u\right)
$$

and

$$
\|\tilde{f}(x)-\tilde{f}(u)\| \leq \mathrm{d}(u, x)=r, \quad\|\tilde{f}(x)-\tilde{f}(v)\| \leq \mathrm{d}(v, x) .
$$

Hence, $\tilde{f}(u) \in C_{1} \cap C_{2}$ and $\tilde{f}(x) \in C$, so that $C_{1} \cap C_{2} \cap\left(C+r Q_{0}\right) \ni \tilde{f}(u)$ proving 5.27).

This enables us to apply Proposition 2.6 to the sets $C_{1}, C_{2}$ and $C$. By this proposition,

$$
\begin{aligned}
\widetilde{A} & =\left[\left\{\left(C_{1} \cap C_{2}\right)+L r Q_{0}\right\} \cap C\right]+\theta(L) \varepsilon Q_{0} \\
& \supset\left[\left(C_{1} \cap C_{2}\right)+(L r+\varepsilon) Q_{0}\right] \cap\left[\left\{\left(C_{1}+r Q_{0}\right) \cap C\right\}+\varepsilon Q_{0}\right] \cap\left[\left\{\left(C_{2}+r Q_{0}\right) \cap C\right\}+\varepsilon Q_{0}\right] \\
& =S_{1} \cap S_{2} \cap S_{3} .
\end{aligned}
$$

Prove that $S_{i} \supset F^{[2]}(y)$ for every $i=1,2,3$. We begin with the set

$$
\begin{aligned}
S_{1} & =\left(C_{1} \cap C_{2}\right)+(L r+\varepsilon) Q_{0} \\
& =\left[\left\{F\left(u^{\prime}\right)+\mathrm{d}\left(u^{\prime}, u\right) Q_{0}\right\} \cap\left\{F\left(u^{\prime \prime}\right)+\mathrm{d}\left(u^{\prime \prime}, u\right) Q_{0}\right\}\right]+(L \mathrm{~d}(u, x)+L \mathrm{~d}(x, y)) Q_{0} .
\end{aligned}
$$

See (5.26). By the triangle inequality, $\mathrm{d}(u, x)+\mathrm{d}(x, y) \geq \mathrm{d}(u, y)$ so that

$$
S_{1} \supset\left[\left\{F\left(u^{\prime}\right)+\mathrm{d}\left(u^{\prime}, u\right) Q_{0}\right\} \cap\left\{F\left(u^{\prime \prime}\right)+\mathrm{d}\left(u^{\prime \prime}, u\right) Q_{0}\right\}\right]+L \mathrm{~d}(u, y) Q_{0}=T_{y}\left(u, u^{\prime}, u^{\prime \prime}\right) .
$$

See 5.11). But, thanks to 3.9$), T_{y}\left(u, u^{\prime}, u^{\prime \prime}\right) \supset F^{[2]}(y)$ proving the required inclusion $S_{1} \supset F^{[2]}(y)$.

Prove that $S_{2} \supset F^{[2]}(y)$. We have

$$
\begin{aligned}
S_{2} & =\left[\left(C_{1}+r Q_{0}\right) \cap C\right]+\varepsilon Q_{0} \\
& =\left[\left\{\left(F\left(u^{\prime}\right)+\mathrm{d}\left(u^{\prime}, u\right) Q_{0}\right)+\mathrm{d}(x, u) Q_{0}\right\} \cap\left\{F(v)+\mathrm{d}(x, v) Q_{0}\right\}\right]+L \mathrm{~d}(x, y) Q_{0} .
\end{aligned}
$$

Therefore, thanks to the triangle inequality, 5.11) and (3.9)

$$
S_{2} \supset\left[\left(F\left(u^{\prime}\right)+\mathrm{d}\left(u^{\prime}, x\right) Q_{0}\right) \bigcap\left(F(v)+\mathrm{d}(x, v) Q_{0}\right)\right]+L \mathrm{~d}(x, y) Q_{0}=T_{y}\left(x, u^{\prime}, v\right) \supset F^{[2]}(y) .
$$

In the same way we show that $S_{3} \supset F^{[2]}(y)$. Hence, $\widetilde{A} \supset S_{1} \cap S_{2} \cap S_{3} \supset F^{[2]}(y)$.

Combining this inclusion with definition (5.25) and representation (5.24), we conclude that $F^{[2]}(x)+\tau Q_{0} \supset F^{[2]}(y)$. By interchanging the roles of $x$ and $y$ we obtain also the inclusion $F^{[2]}(y)+\tau Q_{0} \supset F^{[2]}(x)$. These two inclusions imply inequality

$$
\mathrm{d}_{\mathrm{H}}\left(F^{[2]}(x), F^{[2]}(y)\right) \leq \tau=\tilde{\gamma}(L) \mathrm{d}(x, y)=\lambda_{1} \tilde{\gamma}(L) \rho(x, y)
$$

proving (1.8) with $\gamma=\lambda_{1} L(3 L+1) /(L-1)$. We recall that $L=\lambda_{2} / \lambda_{1}$, so that inequality (1.8) holds with any $\gamma \geq \lambda_{2}\left(3 \lambda_{2}+\lambda_{1}\right) /\left(\lambda_{2}-\lambda_{1}\right)$.

The proof of Theorem 5.5 is complete. 


\section{References}

[1] Z. Artstein, Extension of Lipschitz selections and an application to differential inclusions, Nonlinear Anal. 16 (1991) 701-704.

[2] J.-P. Aubin, H. Frankowska, Set-valued analysis, Systems \& Control: Foundations \& Applications, 2. Birkhauser Boston, 1990.

[3] G. Basso, Computation of maximal projection constants, J. Funct. Anal. 277 (2019) 3560-3585.

[4] Y. Benyamini, J. Lindenstrauss, Geometric nonlinear functional analysis, Vol. 1, in: American Mathematical Society Colloquium Publications, 48. American Mathematical Society, Providence, RI, 2000. xii+488 pp.

[5] Yu. Brudnyi, P. Shvartsman, Generalizations of Whitney's extension theorem, Internat. Math. Res. Notices (1994), no. 3, 129-139.

[6] Yu. Brudnyi, P. Shvartsman, The Whitney problem of existence of a linear extension operator. J. Geom. Anal., 7 (1997), no. 4, 515-574.

[7] Yu. Brudnyi, P. Shvartsman, Whitney Extension Problem for Multivariate $C^{1, \omega}$ functions. Trans. Amer. Math. Soc. 353, No. 6, (2001) 2487-2512.

[8] B. Chalmers, G. Lewicki, A proof of the Grunbaum conjecture, Studia Math. 200 (2010), no. 2, 103-129.

[9] L. Danzer, B. Grünbaum, V. Klee, Helly's Theorem and its relatives. in: AMS Symposium on Convexity, Seattle, Proceedings of Symposium on Pure Mathematics, Vol. 7, Amer. Math. Soc., Providence, RI, 1963, pp. 101-180.

[10] C. Fefferman, A sharp form of Whitney extension theorem, Ann. of Math. 161 (2005), no. 1, 509-577.

[11] C. Fefferman. Whitney extension problem for $C^{m}$. Ann. of Math. 164, no. 1, (2006) 313-359.

[12] C. Fefferman, Whitney extension problems and interpolation of data, Bull. Amer. Math. Soc. 46 (2) (2009) 207-220.

[13] C. Fefferman, A. Israel, Fitting Smooth Functions to Data, CBMS Regional Conference Series in Mathematics, 135. American Mathematical Society, Providence, RI, 2020. xi+160 pp.

[14] C. Fefferman, A. Israel, G. K. Luli, Finiteness principles for smooth selection, Geom. Funct. Anal. 26 (2016), no. 2, 422-477.

[15] C. Fefferman, B. Pegueroles, Efficient algorithms for approximate smooth selection, J. Geom. Anal. 31 (2021), no. 7, 6530-6600.

[16] C. Fefferman, P. Shvartsman, Sharp finiteness principles for Lipschitz selections, Geom. Funct. Anal. 28 (2018) 1641-1705.

[17] B. Grünbaum, Projection constants, Trans. Amer. Math. Soc. 95 (1960) 451465. 
[18] M. D. Kirszbraun, Über die zusammenziehenden und Lipschitzchen Transformationen, Fundam. Math. 22 (1934) 77-108.

[19] K. Przesławski, L. E. Rybinski, Concepts of lower semicontinuity and continuous selections for convex valued multifunctions, J. Approx. Theory 68 (1992) 262-282.

[20] K. Przesławski, D. Yost, Continuity properties of selectors and Michael's theorem, Mich. Math. J. 36 (1989) 113-134.

[21] K. Przesławski, D. Yost, Lipschitz Retracts, Selectors and Extensions, Mich. Math. J. 42 (1995) 555-571.

[22] M. Rieffel, Lipschitz extension constants equal projection constants. Operator theory, operator algebras, and applications, 147-162, Contemp. Math., 414, Amer. Math. Soc., Providence, RI, 2006.

[23] P. Shvartsman, On Lipschitz selections of affine-set valued mappings, Geom. Funct. Anal. 11 (2001), no. 4, 840-868.

[24] P. Shvartsman, Lipschitz selections of set-valued mappings and Helly's theorem, J. Geom. Anal. 12 (2002), no. 2, 289-324.

[25] P. Shvartsman, Barycentric selectors and a Steiner-type point of a convex body in a Banach space, J. Funct. Anal. 210 (2004), no. 1, 1-42.

[26] P. Shvartsman, The Whitney extension problem and Lipschitz selections of set-valued mappings in jet-spaces. Trans. Amer. Math. Soc. 360 (2008), no. 10, 5529-5550.

[27] H. Whitney. Analytic extension of differentiable functions defined in closed sets. Trans. Amer. Math. Soc. 36 (1934) 63-89. 